Estudo de alguns problemas elípticos para o operador biharmônico

\author{
Marcos Tadeu de Oliveira Pimenta
}




\title{
Estudo de alguns problemas elípticos para o operador biharmônico
}

\author{
Marcos Tadeu de Oliveira Pimenta
}

Orientador: Prof. Dr. Sérgio Henrique Monari Soares

Tese apresentada ao Instituto de Ciências Matemáticas e de Computação - ICMC-USP, como parte dos requisitos para obtenção do título de Doutor em Ciências - Matemática. VERSÃO REVISADA

\section{USP - São Carlos}

Maio de 2011 
Ficha catalográfica elaborada pela Biblioteca Prof. Achille Bassi e Seção Técnica de Informática, ICMC/USP, com os dados fornecidos pelo(a) autor(a)

Pimenta, Marcos Tadeu de Oliveira
Estudo de alguns problemas elípticos para o
operador biharmônico / Marcos Tadeu de Oliveira
Pimenta; orientador Sérgio Henrique Monari Soares --
São Carlos, 2011.
93 p.
Tese (Doutorado - Programa de Pós-Graduação em
Matemática)-- Instituto de Ciências Matemáticas e
de Computação, Universidade de São Paulo, 2011.
1. Problemas elípticos biharmônicos. 2. Métodos
variacionais. I. Soares, Sérgio Henrique Monari,
orient. II. Título.


Aos meus pais

Aloísio e Ana, à

minha esposa

Lane e ao meu

filho Enzo 

Agradeço primeiramente à Deus por ter colocado em meu caminho tantas pessoas que me ajudaram e tornaram possível esse trabalho.

Agradeço especialmente ao meu pai Aloísio e a minha mãe Ana, por terem me dado a melhor educação que lhes foi possível oferecer, pelos valores morais e também pelo carinho e amor incondicional que sempre recebi. Mais do que isso, agradeço pelos exemplos de vida, superação e força de vontade que sempre pude colher dentro do meu lar.

Ao meu irmão João Paulo e ao meu primo-irmão Cezar pelo companherismo, amizade e cumplicidade em todos os momentos da minha vida.

Ao meu sogro Moacyr e minha sogra Vera, por terem me oferecido todo o apoio do qual precisei durante essa caminhada.

À minha esposa Lane por seu amor infinito, companheirismo, coragem e disponibilidade em resolver vários problemas sozinha, em benefício da tranquilidade que tanto me foi necessária durante meus estudos.

Ao meu filho Enzo, que mesmo sem ainda ter vindo ao mundo já me proporcionou muitos momentos de alegria.

Aos meus ex-companheiros de república: Juliano (Dedo), Alex (Cabeça), Cláudio, Dusse, Fabrício, Rodrigo (Ursão), Marcus Bronzi (Pink/Nevasca/Mopeta), Juliano Oler (Vôzinho), Jamil (Vôzinho 2), Mário Henrique, Yuri, Everaldo, Flank e Marcos (Japa), por alguns dos momentos mais memoráveis da minha vida. Agradeço também a todos os amigos, tanto da FCT - Unesp de Presidente Prudente, quanto do ICMC - USP, entre eles: Hartmann, Thiago Catalan (Ubarana/Rústico), Kleyber (Baiano), Thaíses Jordão (Rústica) e Monis, John Beiro, Claudinei, Edinho, Suzete, Cléber, André, Jean, Catiana, Fernando Micena e Lucas Felipe, pelos momentos de descontração e amizade.

À todos os meus professores, em especial aos da FCT-Unesp e do ICMC. Agradeço também aos professores Claudianor Alves e Marco Aurélio Souto, pelas várias conversas que tanto contribuiram na elaboração desse trabalho. 
Ao meu orientador Sérgio Henrique Monari Soares, pela maneira responsável e sempre visando o meu melhor, com a qual conduziu esse trabalho. Sua paciência, dedicação e profissionalismo sempre me servirão como modelo ao longo de toda a minha vida.

Por último, agradeço ao $\mathrm{CNPq}$ pelo apoio financeiro. 
Nesse trabalho estudamos questões de existência, multiplicidade e concentração de soluções de uma classe de problemas elípticos biharmônicos. Nos três primeiros capítulos são utilizados métodos variacionais para estudar a existência, multiplicidade e comportamento assintótico das soluções fracas não-triviais de equações de Schrödinger estacionárias biharmônicas com diferentes hipóteses sobre o potencial e sobre a não-linearidade. No último capítulo, o método de decomposição em cones duais é empregado para obter a existência de três soluções (positiva, negativa e nodal) para uma equação biharmônica. 

In this work we study some problems on existence, multiplicity and concentration of solutions of biharmonic elliptic equtions. In the first three chapters, variational methods are used to study the existence, multiplicity and the asymptotic behavior of weak nontrivial solutions of stationary Schrödinger biharmonic equations under certain assumptions on the potential function and the nonlinearity. In the last chapter we use variational methods again and also the dual decomposition method to get existence of positive, negative and sign-changing solutions for a biharmonic equation. 



\section{Índice de Notações}

$|A|$ é a medida de Lebesgue de um subconjunto $A \subset \mathbb{R}^{N}$;

$C^{k}(\Omega)=\{u: \Omega \rightarrow \mathbb{R} ; \quad$ u é continuamente $k$ vezes diferenciável $\}$;

$C_{0}^{k}(\Omega)=\left\{u \in C^{k}(\Omega) ; \operatorname{supp}(u)\right.$ é compacto $\} ;$

$C^{k, \alpha}=\left\{u \in C^{k}(\Omega) ; \quad D^{k} u\right.$ é $\alpha$-Hölder contínua $\}$;

$\|u\|_{L^{p}(\Omega)}=\left(\int_{\Omega}|u|^{p} d x\right)^{\frac{1}{p}}$

$L^{p}(\Omega)=\left\{u: \Omega \rightarrow \mathbb{R} ; \quad\right.$ u é mensurável e $\left.\|u\|_{L^{p}(\Omega)}<\infty\right\} ;$

$\|u\|_{L^{\infty}(\Omega)}=\inf \{a \geq 0 ; \quad|\{x \in \Omega ;|u(x)|>a\}|=0\} ;$

$L^{\infty}(\Omega)=\left\{u: \Omega \rightarrow \mathbb{R} ; \quad\right.$ u é mensurável e $\left.\|u\|_{L^{\infty}(\Omega)}<\infty\right\}$;

$W^{m, p}(\Omega)=\left\{u \in L^{p}(\Omega) ; \quad D^{\alpha} u \in L^{p}(\Omega) \quad \forall|\alpha| \leq m\right\}$, onde $\alpha=\left(\alpha_{1}, \ldots, \alpha_{N}\right)$ é um multi-índice;

$\|u\|_{W^{m, p}(\Omega)}=\left(\sum_{i=1}^{m}\left\|D^{i} u\right\|_{L^{p}(\Omega)}\right)^{\frac{1}{p}}$;

$W_{0}^{m, p}(\Omega)=\overline{C_{0}^{\infty}(\Omega)}$, onde o fecho é tomado com relação a norma $\|\cdot\|_{W^{m, p}(\Omega)}$;

$H^{m}(\Omega)=W^{2, m}(\Omega)$;

$H_{0}^{m}(\Omega)=\overline{C_{0}^{\infty}(\Omega)}$, onde o fecho é tomado com relação a norma $\|\cdot\|_{H^{m}(\Omega)}$;

$\mathcal{S}=\left\{u \in C^{\infty}\left(\mathbb{R}^{N}\right) ; \quad \sup _{x \in \mathbb{R}^{N}}\left(1+|x|^{k}\right)\left|D^{\alpha} u\right|<\infty, \forall k \in \mathbb{N}\right.$ e $\alpha$ um multi-índice $\} ;$

$\mathcal{S}^{\prime}$ é o espaço das distribuições temperadas (distribuições com suporte compacto);

$\widehat{u}$ é a transformada de Fourier da função $u \in \mathcal{S}$;

$\Delta u=\sum_{i=1}^{N} \frac{\partial^{2} u}{\partial x_{i}^{2}}$

$\Delta_{p} u=\operatorname{div}\left(|\nabla u|^{p-2} \nabla u\right)$;

$\Delta^{2} u=\Delta(\Delta u)$

$u^{+}(x)=\max \{u(x), 0\}$

$u^{-}(x)=\min \{u(x), 0\}$;

$2_{*}=\frac{2 N}{N-4}$ é o expoente crítico de Sobolev para a imersão de $H^{2}(\Omega)$ em $L^{q}(\Omega)$;

$\operatorname{cat}(\Omega)$ é a categoria de Lusternik-Schnirelman do conjunto $\Omega$. 

Agradecimentos $\quad$ iii

Resumo v v

Abstract vii

Índice de Notações $\quad$ ix

$\begin{array}{ll}\text { Introdução } & 1\end{array}$

1 Existência e concentração de soluções: hipótese global sobre o potencial $\quad 7$

1.1 Introdução . . . . . . . . . . . . . . . . . . . . . . . 7

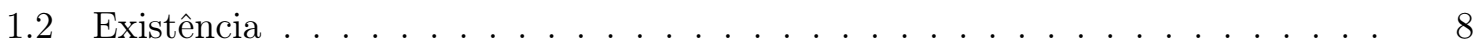

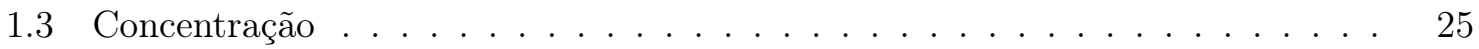

1.4 Estimativa $L^{\infty} \ldots \ldots \ldots \ldots \ldots \ldots \ldots \ldots \ldots$

2 Existência e concentração de soluções: hipótese local sobre o potencial 39

2.1 Introdução . . . . . . . . . . . . . . . . . . . . . . . . . . . . . 39

2.2 O problema modificado . . . . . . . . . . . . . . . . . . 40

2.3 Decaimento uniforme . . . . . . . . . . . . . . . . . . 54

3 Multiplicidade de soluções via categoria de Lusternik-Schnirelman $\quad 57$

3.1 Introdução . . . . . . . . . . . . . . . . . . . . . . . 57

3.2 Condição de compacidade . . . . . . . . . . . . . . . . . . . . 58

3.3 Comportamento dos níveis $\operatorname{minimax} \ldots \ldots \ldots$. . . . . . . . 66

3.4 Demonstração do Teorema $3.1 .1 \ldots \ldots \ldots$. . . . . . . . . . . . 73

4 Existência de soluções nodais para uma equação biharmônica $\quad \mathbf{7 5}$

4.1 Método de decomposição em cones duais . . . . . . . . . . . . . . . . 76 


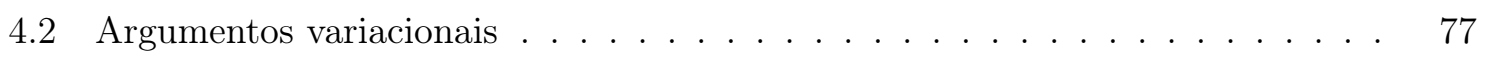

$\begin{array}{ll}\text { Referências Bibliográficas } & 91\end{array}$ 


\section{Introdução}

Nos últimos anos, vários autores têm estudado diversas questões relativas à equação de Schrödinger estacionária

$$
\left\{\begin{array}{r}
-\epsilon^{2} \Delta u+V(x) u=f(u) \text { em } \Omega, \\
u \in H^{1}(\Omega),
\end{array}\right.
$$

com condições de fronteira de Neumann ou Dirichlet, onde $\Omega \subset \mathbb{R}^{N}$ é um domínio não necessariamente limitado. Motivado por Floer e Weinstein [20], Rabinowitz em [30] usou argumentos do tipo passo da montanha para encontrar soluções de energia mínima de (1) para $\epsilon>0$ suficientemente pequeno, onde $N \geq 3, \Omega=\mathbb{R}^{N}$ e $f$ é uma não-linearidade superlinear e subcrítica. A função $V$ é um potencial não-negativo satisfazendo a seguinte condição

$$
0<V_{0}=\inf _{x \in \mathbb{R}^{N}} V(x)<\liminf _{|x| \rightarrow \infty} V(x) .
$$

Em [35], Wang provou que as soluções do passo da montanha encontradas por Rabinowitz em [30] apresentam um fenômeno de concentração em torno de um mínimo global de $V$, se $\epsilon \rightarrow 0$. Em [18], del Pino e Felmer usaram um método de penalização para provar a existência e concentração de soluções para o problema (1), com não-linearidade superlinear e subcrítica e com o potencial $V$ satisfazendo a seguinte condição

$$
\inf _{x \in \Lambda} V(x)<\inf _{x \in \partial \Lambda} V(x),
$$

onde $\Lambda$ é um domínio limitado contido em $\Omega$. Esses argumentos inspiraram muitos autores nos últimos anos, entre eles Alves e Figueiredo em [3,6] consideraram o problema (1) com o operador de Laplace substituído pelo p-Laplaciano e obtiveram existência, multiplicidade e concentração de soluções positivas. Podemos citar ainda os trabalhos [19] e [4], onde os autores obtiveram multiplicidade e concentração de soluções positivas e nodais, respectivamente, para uma equação relacionada a (1). Em [11], Alves e Soares provaram a existência e concentração 
de soluções nodais de (1), para o caso em que $N=2$ e $f$ apresenta crescimento exponencial crítico.

Embora muitos autores tenham estudado o problema (1) para os operadores Laplaciano e p-Laplaciano, muito poucos trabalhos podem ser encontrados tratando de equações de Schrödinger biharmônicas ou mesmo poliharmônicas. Entre eles podemos citar [7] e [8], nos quais provou-se a existência de soluções não-triviais para problemas biharmônicos semilineares com não-linearidades com crescimento crítico. Podemos citar ainda [32] onde obteve-se infinitas soluções para um problema poliharmônico em domínios ilimitados.

Vale ressaltar que, embora o operador biharmônico seja linear e permita que se trabalhe em espaços de Hilbert, não se deve pensar que todos os argumentos utilizados em resultados sobre operadores de segunda ordem, tanto semilinear quanto quasilinear, se adaptam trivialmente ao operador biharmônico. De fato, resultados envolvendo o operador biharmônico apresentam várias dificuldades de ordem técnica, devido a falta de resultados fundamentais frequentemente utilizados em problemas de segunda ordem. Entre esses, podemos citar a falta, em geral, de um princípio do máximo forte, de desigualdades do tipo Harnack e a não-positividade, em geral, da função de Green do operador biharmônico. Ainda mais, apenas o fato de não ser verdade em geral que, se $u \in H^{2}(\Omega)$, então $|u|, u^{+}, u^{-} \in H^{2}(\Omega)$, onde $u^{+}=\max (u, 0)$ e $u^{-}=\min (u, 0)$, representa uma enorme dificuldade para se provar resultados de positividade de soluções, bem como a existência de soluções nodais para equações biharmônicas. Também argumentos baseados no método de iteração de Moser, são bastante difíceis de se implementar para esse operador. Por último, resultados que utilizam-se da simetrização de Schwarz $u^{*}$ de funções de $H^{2}(\Omega)$ não podem ser utilizados em geral devido ao fato de $u^{*} \notin H^{2}(\Omega)$, mesmo em casos em que $u$ é bastante suave.

Motivados por essas dificuldades e pelos trabalhos citados, vamos nesse texto estender no Capítulo 1 os resultados de [30] e [35] e, no Capítulo 2, os resultados de [18], para a seguinte classe de problemas elípticos biharmônicos

$$
\left\{\begin{aligned}
\epsilon^{4} \Delta^{2} u+V(x) u= & f(u) \text { em } \mathbb{R}^{N} \\
& u \in H^{2}\left(\mathbb{R}^{N}\right),
\end{aligned}\right.
$$

onde $\Delta^{2} u=\Delta(\Delta u), \epsilon>0, N \geq 5$ e a não-linearidade $f: \mathbb{R} \rightarrow \mathbb{R}$ é uma função sublinear na origem, com crescimento superlinear e subcrítico no infinito. No Capítulo 1, para superar a perda de compacidade no problema, vamos supor que $V$ satisfaz a condição (2) de modo que, usando argumentos variacionais do tipo passo da montanha, obtenhamos soluções para o problema (4), para valores de $\epsilon$ suficientemente pequenos. Nesse capítulo, vamos supor que a não-linearidade $f$ satisfaz uma condição de superlinearidade mais fraca que a seguinte versão da condição de Ambrosetti-Rabinowitz:

(AR) Existe $\eta>2$ tal que

$$
0<\eta F(s) \leq f(s) s, \quad \text { para todo } s \neq 0,
$$


onde $F(s)=\int_{0}^{s} f(t) d t$. Isso vai representar uma certa dificuldade para provar que as sequências de Palais-Smale são limitadas, o que será feito utilizando-se argumentos empregados em [27], e também para se provar que a Variedade de Nehari associada ao problema é homeomorfa à bola unitária, o que será feito adaptando-se argumentos de Weth e Szulkin em [33]. Para a concentração, vamos fazer uma análise cuidadosa dos níveis de energia minimax das soluções obtidas, de forma a provar um decaimento uniforme de certas translações das mesmas. Isto, como veremos, implicará na concentração das soluções em torno de um ponto de mínimo do potencial $V$ em $\mathbb{R}^{N}$.

No Capítulo 2, explorando uma condição local sobre o potencial $V$ tal como em [18], faremos uma modificação da não-linearidade de modo que a condição de Palais-Smale seja válida para o funcional modificado associado e assim utilizar o Teorema do Passo da Montanha para obter as soluções do problema modificado. Nesse capítulo, a exemplo do Capítulo 1, vamos considerar para a não-linearidade uma condição de superlinearidade mais fraca que a condição $(A R)$. Nesse sentido, uma importante referência que trata de problemas elípticos de segunda ordem singularmente perturbados e estuda existência e concentração de soluções, é o trabalho de Jeanjean e Tanaka [22]. Tal como no Capítulo 1, faremos uma análise dos níveis de energia minimax e utilizaremos ainda resultados de compacidade em variedades de Nehari para provar novamente o decaimento de certas translações das soluções, o que implicará não só na presença do fenômeno de concentração, bem como no fato de as soluções do problema modificado serem de fato soluções do problema original. Vale ressaltar que, embora alguns argumentos utilizados sejam semelhantes àqueles utilizados por del Pino e Felmer em [18], a abordagem teve de ser significativamente modificada devido a falta de um princípio do máximo para o operador biharmônico. Neste sentido, alguns argumentos utilizados em [3] e [9] foram bastante úteis, bem como uma estimativa $L^{\infty}$ para soluções de problemas biharmônicos subcríticos estabelecida por Ramos em [31].

No Capítulo 3 vamos estudar questões sobre existência e multiplicidade de soluções para a seguinte classe de problemas

$$
\left\{\begin{array}{l}
\epsilon^{4} \Delta^{2} u+(\lambda V(x)+1) u=f(u) \text { em } \mathbb{R}^{N} \\
u \in H^{2}\left(\mathbb{R}^{N}\right),
\end{array}\right.
$$

onde $\epsilon$ e $\lambda$ são parâmetros positivos. Ao longo dos últimos anos, o problema (5) foi objeto de estudo de vários autores. Em [13], Bartsch e Wang estudaram um problema semelhante a (5), $\operatorname{com} \epsilon=1$, e considerando o operador $\Delta$. Questões como existência, multiplicidade e o comportamento assintótico de soluções, quando o parâmetro $\lambda \rightarrow \infty$ foram abordadas. Lá, os autores concluíram que se $\lambda_{n} \rightarrow \infty$, então existe uma sequência de soluções $\left(u_{n}\right) \in H^{1}\left(\mathbb{R}^{N}\right)$ que converge ao longo de uma subsequência para uma solução de energia mínima do seguinte problema limite

$$
\begin{cases}\Delta u+u & =f(u) \text { em } \Omega \\ u & =0 \text { em } \partial \Omega\end{cases}
$$


sendo $\Omega=\operatorname{int}\left(V^{-1}(0)\right)$. Além disso, demonstraram que se $\lambda>0$ é suficientemente grande, então o problema (5) tem ao menos $\operatorname{cat}(\Omega)$ soluções, onde $\Omega=i n t V^{-1}(0)$ e cat simboliza a categoria de Lusternik-Schnirelman. Motivados por esse e outros trabalhos, Alves e Soares em [12] usaram métodos variacionais para provar a existência e multiplicidade de soluções positivas para o problema (5), porém considerando o operador $\Delta_{p}$, quando para valores suficientemente grandes de $\lambda$ e suficientemente pequenos de $\epsilon$. Esse trabalho nos motivou a estender esses resultados para o operador $\Delta^{2}$. Assim, o principal resultado demonstrado no Capítulo 3 é o seguinte, em que deixamos para o Capítulo 3 o enunciado das hipóteses que $f$ e $V$ devem satisfazer.

Teorema 0.0.1. Existe $\epsilon^{*}>0$ tal que, para todo $\epsilon \in\left(0, \epsilon^{*}\right)$, existe $\Lambda(\epsilon)>0$ tal que (5) tem pelo menos cat $(\bar{\Omega})$ soluções fracas não-triviais, sempre que $\lambda \geq \Lambda(\epsilon)$.

No Capítulo 4, tratamos da difícil tarefa de estudar a existência de soluções nodais para equações biharmônicas. Como mencionado anteriormente, tal dificuldade surge da impossibilidade, em geral, da decomposição de uma função $u \in H^{2}\left(\mathbb{R}^{N}\right)$ na forma $u=u^{+}+u^{-}$, o que é absolutamente trivial para funções de $H^{1}\left(\mathbb{R}^{N}\right)$. Uma vez que para equações de segunda ordem essa decomposição desempenha um papel fundamental na maioria dos argumentos utilizados para obtenção de soluções, quando tratando de equações de quarta ordem, os métodos utilizados são bastante limitados por tal dificuldade. Em [36], Weth estudou a existência de soluções para o seguinte problema biharmônico

$$
\Delta^{2} u=f(x, u) \quad \text { em } \Omega,
$$

com condições de fronteira do tipo Dirichlet $\left(u=\frac{\partial u}{\partial \eta}=0\right)$ ou Navier $(u=\Delta u=0)$, onde $\Omega \subset \mathbb{R}^{N}$ é um domínio suave e $f$ é uma não-linearidade superlinear e subcrítica. Nesse trabalho, o autor obteve três soluções, sendo duas com sinal definido e uma outra que muda de sinal, através do estudo da dinâmica da equação ordinária induzida pelo campo negativo do gradiente do funcional energia associado. Para a distinção entre as soluções, Weth usou fortemente o Método de Decomposição em Cones Duais, desenvolvido por J. J. Moreau em [28], que consiste na decomposição de uma função de um espaço de Hilbert $H$ em duas outras, sendo que cada uma delas pertence a um cone em $H$. Dependendo do subespaço de $H^{2}(\Omega)$ com o qual se trabalha, é possível usar esse método para decompor uma função $u \in H^{2}(\Omega)$ em duas outras sendo uma positiva e outra negativa. Portanto, esse método pode, em alguns casos, substituir a decomposição usual $u=u^{+}+u^{-}$de funções de $H^{1}(\Omega)$. Em [24], Liu e Huang utilizaram-se de uma abordagem semelhante à empregada por Weth em [36] para estudar o seguinte problema com condições de fronteira de Navier

$$
\left\{\begin{array}{r}
\Delta^{2} u+c \Delta u=f(x, u) \quad \text { em } \Omega \\
u=\Delta u=0 \quad \text { em } \partial \Omega .
\end{array}\right.
$$

Seguindo as idéias de Weth em [36], no Capítulo 4 vamos estudar a existência de três soluções, sendo uma positiva, uma negativa e outra que muda de sinal, para (7) com condição 
de fronteira de Navier, onde $\Omega$ é um domínio suave e limitado de $\mathbb{R}^{N}$. Nossa contribuição será no sentido de estender o resultado para não-linearidade $f$ satisfazendo uma condição de superlinearidade mais fraca que a condição de Ambrosetti-Rabinowitz. Mais especificamente, consideramos o seguinte conjunto de hipóteses em $f$ :

i)

$$
\lim _{|s| \rightarrow \infty} \frac{F(x, s)}{s^{2}}=+\infty
$$

uniformemente para quase todo $x$ em $\Omega$;

ii) existe $C>0$ tal que $H(x, t) \leq H(x, s)+C$, para $0<t<s$ ou $s<t<0, x \in \Omega$, em que $H(x, t)=f(x, t) t-2 F(x, t)$.

Os argumentos implementados para se obter compacidade, mesmo sem a condição $(A R)$, foram inspirados nos resultados de Miyagaki e Souto em [27].

Por fim, motivados pelos resultados em [5] sobre a existência de soluções to tipo multipeak para (1), gostaríamos de mencionar que houve uma tentativa de utilizar as mesmas técnicas empregadas no Capítulo 2 para obter esse tipo de solução para o problema (4), no caso em que o potencial satisfaz a condição local (3) num certo número de subconjuntos de $\mathbb{R}^{N}$. Essa tentativa, porém, foi frustrada pela dificuldade em provar que as soluções do problema modificado pela penalização de fato são soluções do problema original. Isso se deve ao problema causado pela necessidade de lidar simultaneamente com os vários problemas limites que se apresentam. Contudo, consideramos que esse tema é promissor e será nosso objeto de estudo futuro. Notamos que a eventual existência de uma família de soluções do tipo multi-peak para (4) fornece um resultado de múltiplas soluções para esse problema. Portanto, diante da nossa dificuldade e da natureza do problema, entendemos que o resultado apresentado no Capítulo 3 constitui-se numa alternativa para esta questão. 



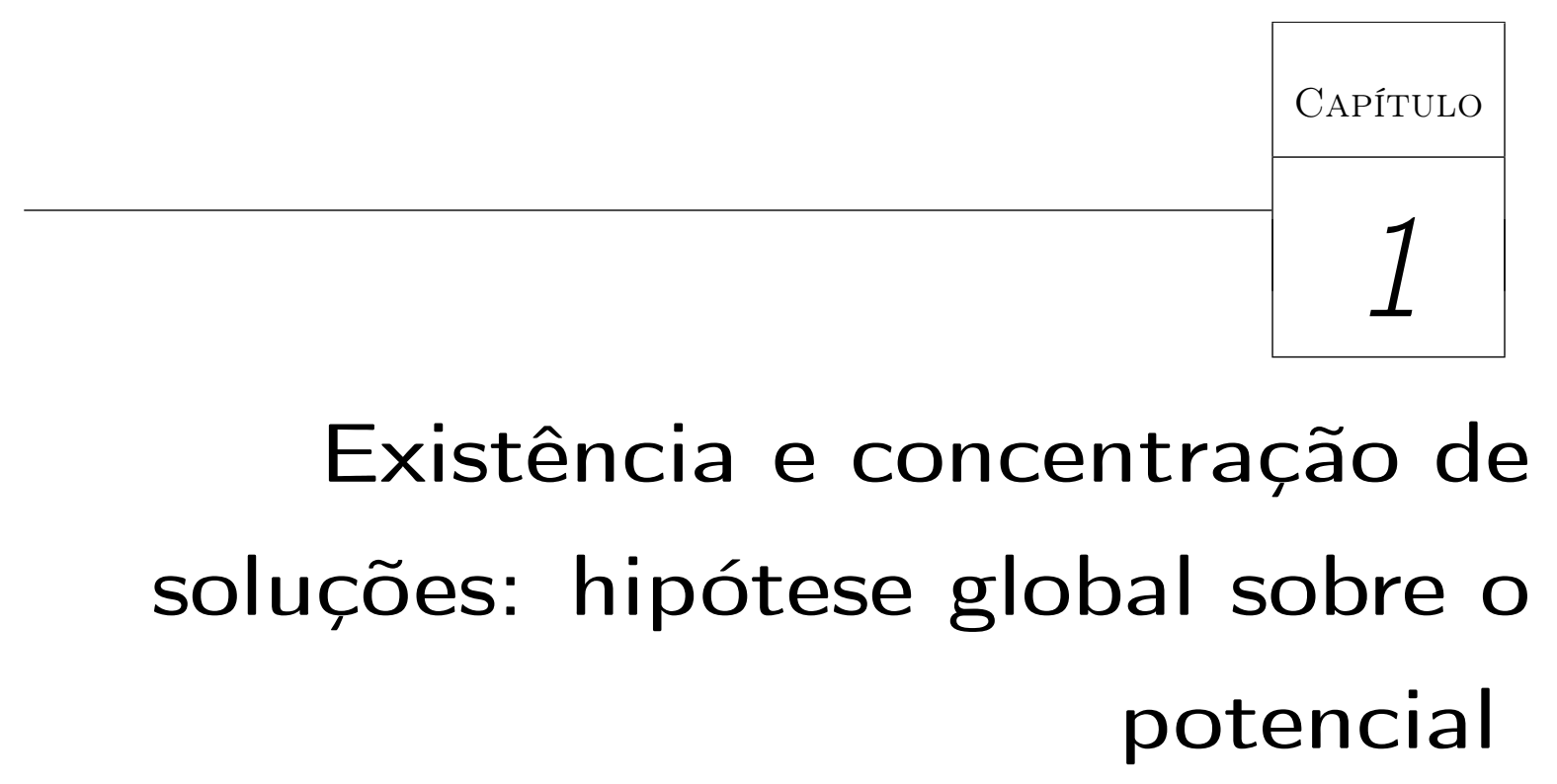

\subsection{Introdução}

Neste capítulo estamos interessados em estudar questões sobre existência e comportamento assintótico de soluções do seguinte problema elíptico

$$
\left\{\begin{array}{l}
\epsilon^{4} \Delta^{2} u+V(x) u=f(u) \text { em } \mathbb{R}^{N} \\
u \in H^{2}\left(\mathbb{R}^{N}\right),
\end{array}\right.
$$

quando $\epsilon>0$, sendo $N \geq 5$ e a não-linearidade $f$ do tipo potência, com crescimento superlinear e subcrítico. O potencial $V$ é positivo e satisfaz uma hipótese global. Mais especificamente, consideremos o seguinte conjunto de hipóteses sobre $f$ e $V$ :

$\left(V_{1}\right) V \in C^{0}\left(\mathbb{R}^{N}\right) \cap L^{\infty}\left(\mathbb{R}^{N}\right)$,

$\left(V_{2}\right) 0<V_{0}:=\inf _{\mathbb{R}^{N}} V<\liminf _{|x| \rightarrow \infty} V(x)=: V_{\infty}$,

$\left(f_{1}\right) f \in C^{1}(\mathbb{R})$,

$\left(f_{2}\right) f(0)=f^{\prime}(0)=0$, 
$\left(f_{3}\right)$ existem constantes $c_{1}, c_{2}>0$ e $p \in\left(1,2_{*}-1\right)$, tais que $|f(s)| \leq c_{1}|s|+c_{2}|s|^{p}$, para todo $s \in \mathbb{R}$, onde $2_{*}=\frac{2 N}{N-4}$,

$\left(f_{4}\right) \lim _{|s| \rightarrow \infty} \frac{F(s)}{s^{2}}=+\infty$, onde $F(s)=\int_{0}^{s} f(t) d t$,

$\left(f_{5}\right) \frac{f(s)}{s}$ é crescente para $s>0$ e decrescente para $s<0$.

Observação 1.1.1. Observe que a hipótese $\left(f_{5}\right)$ implica $h^{\prime}(s)>0$ para $s>0$, onde $h(s)=\frac{f(s)}{s}$. Isto por sua vez implica

$$
f^{\prime}(s) s^{2}-f(s) s>0
$$

para todo $s>0$. Disto segue ainda que $H(s):=f(s) s-2 F(s)$ é crescente para $s>0$ e decrescente para $s<0$. Em particular $H(s) \geq 0$ para todo $s \in \mathbb{R}$.

Não há perda de generalidade em se supor que $V(0)=V_{0}$, uma vez que se esse não for o caso, podemos considerar o problema (1.1) com o potencial $V$ substituído por $W(x)=V\left(x-x_{0}\right)$, onde $V\left(x_{0}\right)=V_{0}$ e dessa forma, transferir os resultados para a equação original através de uma mudança de variável devido à invariância por translação.

Dividimos este capítulo em três seções. Na primeira tratamos do problema de existência de soluções para (1.1), enquanto que na segunda estudamos o comportamento assintótico de uma sequência de soluções de (1.1), quando o parâmetro $\epsilon$ tende à zero. Na terceira, baseados em argumentos de [31], vamos estabelcer uma estimativa $L^{\infty}$ para as soluções encontradas, que será essencial para provar que a família de soluções apresenta o fenômeno de concentração.

\subsection{Existência}

Nessa seção desejamos provar o seguinte resultado.

Teorema 1.2.1. Supondo as hipóteses $\left(V_{1}\right),\left(V_{2}\right)$ sobre o potencial e $\left(f_{1}\right)$ - $\left(f_{5}\right)$ sobre a nãolinearidade, existe $\epsilon_{0}>0$ tal que o problema (1.1) tem solução fraca não-trivial se $0<\epsilon<\epsilon_{0}$.

Antes de iniciar a prova do teorema, vamos introduzir uma série de notações e resultados auxiliares, que serão utilizados por todo o resto do capítulo.

Observe que a equação (1.1) é equivalente à equação

$$
\left\{\begin{array}{l}
\Delta^{2} v+V_{\epsilon}(x) v=f(v) \text { em } \mathbb{R}^{N} \\
v \in H^{2}\left(\mathbb{R}^{N}\right),
\end{array}\right.
$$

onde $V_{\epsilon}(x)=V(\epsilon x)$. Note que o potencial $V_{\epsilon}$ satisfaz as mesmas hipóteses que $V$ e que as soluções $u_{\epsilon}$ de (1.1) e $v_{\epsilon}$ de (1.3) estão relacionadas por

$$
u_{\epsilon}(\epsilon x)=v_{\epsilon}(x) .
$$


O espaço natural para se tratar a equação (1.1), ou equivalentemente (1.3), é o espaço de Sobolev $H^{2}\left(\mathbb{R}^{N}\right)$, que tem estrutura Hilbertiana quando munido do seguinte produto interno

$$
\langle u, v\rangle_{\epsilon}=\int_{\mathbb{R}^{N}}\left(\Delta u \Delta v+V_{\epsilon}(x) u v\right) d x
$$

que por sua vez dá origem a seguinte norma

$$
\|u\|_{\epsilon}^{2}=\int_{\mathbb{R}^{N}}\left(|\Delta u|^{2}+V_{\epsilon}(x) u^{2}\right) d x .
$$

Observação 1.2.2. Uma vez que o potencial é positivo e $V_{\epsilon} \in L^{\infty}\left(\mathbb{R}^{N}\right)$, essa norma é equivalente aquela usual de $H^{2}\left(\mathbb{R}^{N}\right)$.

Seja então

$$
E_{\epsilon}=\left(H^{2}\left(\mathbb{R}^{N}\right),\langle\cdot, \cdot\rangle_{\epsilon}\right) .
$$

Pelo crescimento subcrítico de $f$ descrito na hipótese $\left(f_{3}\right)$, vemos que está bem definido em $E_{\epsilon}$ o funcional energia associado ao problema (1.3), dado por

$$
I_{\epsilon}(u)=\frac{1}{2} \int_{\mathbb{R}^{N}}\left(|\Delta u|^{2}+V_{\epsilon}(x) u^{2}\right) d x-\int_{\mathbb{R}^{N}} F(u) d x,
$$

onde $F(s)=\int_{0}^{s} f(t) d t$.

Ainda pelo crescimento subcrítico de $f$, o funcional $I_{\epsilon} \in C^{1}\left(E_{\epsilon}, \mathbb{R}\right)$.

Usando as hipóteses sobre a não-linearidade $f$, verifica-se que o funcional $I_{\epsilon}$ verifica as hipóteses geométricas do Teorema do Passo da Montanha. Mais especificamente:

Lema 1.2.3. Existe $\rho, r>0$ e $v \in E_{\epsilon}$, com $\|v\|_{\epsilon}>r$, tais que

i) $I_{\epsilon}(u) \geq \rho$ para todo $\|u\|_{\epsilon}=r$;

ii) $I_{\epsilon}(v)<0$.

Demonstração. Por $\left(f_{2}\right)$, dado $\eta>0$ existe $\delta>0$ tal que $|F(s)|<\eta|s|^{2}$ se $|s|<\delta$. Se $|s| \geq \delta$, por $\left(f_{3}\right)$ temos que

$$
\begin{aligned}
|F(s)| & \leq \tilde{c}_{1}|s|^{2}+\tilde{c}_{2}|s|^{p+1} \\
& =\left(\frac{\tilde{c}_{1}}{|s|^{p-1}}+\tilde{c}_{2}\right)|s|^{p+1} \\
& \leq\left(\frac{\tilde{c}_{1}}{\delta^{p-1}}+\tilde{c}_{2}\right)|s|^{p+1} \\
& =A_{\eta}|s|^{p+1}
\end{aligned}
$$

Então

$$
|F(s)| \leq \eta|s|^{2}+A_{\eta}|s|^{p+1} \quad \forall s \in \mathbb{R} .
$$


10 Capítulo 1 - Existência e concentração de soluções: hipótese global sobre o potencial

Logo, pelas imersões de Sobolev, existe uma constante positiva $C(\eta)$ tal que

$$
\int_{\mathbb{R}^{N}}|F(u)| d x \leq \eta\|u\|_{\epsilon}^{2}+C(\eta)\|u\|_{\epsilon}^{p+1}
$$

Assim,

$$
I_{\epsilon}(u) \geq\left[\left(\frac{1}{2}-\eta\right)-C(\eta)\|u\|_{\epsilon}^{p-1}\right]\|u\|_{\epsilon}^{2} .
$$

Portanto, fixando $\eta \in(0,1 / 2)$, existe $r>0$ suficientemente pequeno de modo que

$$
I_{\epsilon}(u) \geq \rho>0, \quad \text { para todo }\|u\|_{\epsilon}=r,
$$

onde $\rho=\left[(1 / 2-\eta)-C(\eta) r^{p-1}\right] r^{2}$, o que verifica $\left.i\right)$.

Para provar ii), fixemos $u \in C_{0}^{\infty}\left(\mathbb{R}^{N}\right)$ e $u>0$. Usando $\left(f_{4}\right)$, dado $M \geq\|u\|_{\epsilon}^{2} / 2\|u\|_{L^{2}}^{2}$, existe $\delta>0$, tal que $F(s) \geq M|s|^{2}$, para todo $s>\delta>0$. Dessa forma,

$$
F(s) \geq M|s|^{2}-c_{0}, \quad \text { para } s \in \mathbb{R}
$$

onde $c_{0}=\max \{F(s) ; 0 \leq s \leq \delta\}$. Assim,

$$
\begin{aligned}
I_{\epsilon}(t u) & =\frac{t^{2}}{2}\|u\|_{\epsilon}^{2}-\int_{\mathbb{R}^{N}} F(t u) d x \\
& \leq \frac{t^{2}}{2}\|u\|_{\epsilon}^{2}-t^{2} M \int_{\mathbb{R}^{N}}|u|^{2} d x+c_{0}|\operatorname{supp}(u)| \\
& =t^{2}\left(\frac{\|u\|_{\epsilon}^{2}}{2}-M \int_{\mathbb{R}^{N}}|u|^{2} d x\right)+c_{0}|\operatorname{supp}(u)| .
\end{aligned}
$$

Portanto, $I_{\epsilon}(t u) \rightarrow-\infty$, quando $t \rightarrow+\infty$ e a prova do lema está completa.

O lema anterior nos habilita a usar o Teorema do Passo da Montanha para definir, para cada $\epsilon>0$, o valor minimax $c_{\epsilon}$ dado por

$$
c_{\epsilon}=\inf _{g \in \Gamma_{\epsilon}} \sup _{0 \leq t \leq 1} I_{\epsilon}(g(t))
$$

onde

$$
\Gamma_{\epsilon}=\left\{g \in C\left([0,1], E_{\epsilon}\right) ; g(0)=0 \text { e } I_{\epsilon}(g(1))<0\right\} .
$$

Visando encontrar soluções de energia mínima, consideremos a variedade de Nehari associada ao problema (1.3), dada por

$$
\mathcal{N}_{\epsilon}=\left\{u \in E_{\epsilon} \backslash\{0\} ; \quad I_{\epsilon}^{\prime}(u) u=0\right\},
$$

ou equivalentemente

$$
\mathcal{N}_{\epsilon}=\left\{u \in E_{\epsilon} \backslash\{0\} ; \quad \int_{\mathbb{R}^{N}}\left(|\Delta u|^{2}+V_{\epsilon}(x) u^{2}\right) d x=\int_{\mathbb{R}^{N}} f(u) u d x\right\}
$$


Note que para cada $u \in E_{\epsilon} \backslash\{0\}$, existe um único valor $\varphi_{\epsilon}(u)>0$ tal que

$$
\left.\frac{d}{d t} I_{\epsilon}(t u)\right|_{t=\varphi_{\epsilon}(u)}=0
$$

De fato,

$$
\begin{aligned}
\frac{d}{d t} I_{\epsilon}(t u)=0 & \Leftrightarrow I_{\epsilon}^{\prime}(t u) u=0 \\
& \Leftrightarrow\|u\|_{\epsilon}^{2}=\int_{\mathbb{R}^{N}} \frac{f(t u) u}{t} d x .
\end{aligned}
$$

Para qualquer $s \neq 0$, por $\left(f_{5}\right)$, a função $t \mapsto \frac{f(t s) s}{t}$ é crescente para $t>0$ e por $\left(f_{2}\right)$, a função pode ser estendida continuamente como 0 para $t=0$. Logo, existe um único $\varphi_{\epsilon}(u)$ tal que $\left.\frac{d}{d t} I_{\epsilon}(t u)\right|_{t=\varphi_{\epsilon}(w)}=0$.

Observação 1.2.4. Da unicidade de $\varphi_{\epsilon}(u)$ para cada $u \neq 0$ e da geometria do funcional $I_{\epsilon}$, podemos concluir que

- $\mathcal{N}_{\epsilon}=\left\{\varphi_{\epsilon}(u) u ; u \in E_{\epsilon} \backslash\{0\}\right\}$.

- $\varphi_{\epsilon}(u)$ é um ponto de máximo para $t \mapsto I_{\epsilon}(t u)$.

Visando caracterizar de uma maneira mais conveniente o nível minimax $c_{\epsilon}$, vamos mostrar que a variedade de Nehari $\mathcal{N}_{\epsilon}$ é homeomorfa à esfera $S_{1}(0)=\left\{u \in E_{\epsilon} ;\|u\|_{\epsilon}=1\right\}$. Para isso, o lema seguinte será fundamental.

Lema 1.2.5. Existe $\delta>0$ tal que $\varphi_{\epsilon}(w) \geq \delta$, para todo $w \in S_{1}(0)$. Além disso, para cada subconjunto compacto $\mathcal{A} \subset S_{1}(0)$, existe $C_{\mathcal{A}}>0$ tal que $\varphi_{\epsilon}(w) \leq C_{\mathcal{A}}$, para todo $w \in \mathcal{A}$.

Demonstração. Mostremos primeiramente que, dado $\mathcal{A} \subset E_{\epsilon} \backslash\{0\}$ compacto e $\left(u_{n}\right) \subset \mathcal{A}$, então:

$$
\lim _{n \rightarrow \infty} \int_{\mathbb{R}^{N}} \frac{F\left(s_{n} u_{n}\right)}{s_{n}^{2}} d x=+\infty
$$

para toda sequência $s_{n} \rightarrow+\infty$. Note que $u_{n} \rightarrow u \in \mathcal{A}$ em $E_{\epsilon}$ a menos de subsequência e ainda $u \neq 0$.

Seja $s_{n} \rightarrow 0$. Denote por $\Gamma=\left\{x \in \mathbb{R}^{N} ; u(x) \neq 0\right\}$. Pelo Lema de Fatou e por $\left(f_{4}\right)$,

$$
\begin{aligned}
\lim _{n \rightarrow \infty} \int_{\mathbb{R}^{N}} \frac{F\left(s_{n} u_{n}\right)}{s_{n}^{2}} d x & =\lim _{n \rightarrow \infty} \int_{\mathbb{R}^{N}} \frac{F\left(s_{n} u_{n}\right)}{s_{n}^{2} u_{n}^{2}} u_{n}^{2} d x \\
& \geq \lim _{n \rightarrow \infty} \int_{\Gamma} \frac{F\left(s_{n} u_{n}\right)}{s_{n}^{2} u_{n}^{2}} u_{n}^{2} d x=+\infty
\end{aligned}
$$

Provemos agora que existe $\delta>0$ tal que $\varphi_{\epsilon}(w) \geq \delta$, para todo $w \in S_{1}(0)$. De fato, supondo o contrário, existiria $\left(w_{n}\right) \subset S_{1}(0)$ tal que $\varphi_{\epsilon}\left(w_{n}\right) \rightarrow 0$ quando $n \rightarrow \infty$. Então

$$
0=\left.\frac{d}{d t} I\left(t w_{n}\right)\right|_{t=\varphi_{\epsilon}\left(w_{n}\right)}=I_{\epsilon}^{\prime}\left(\varphi_{\epsilon}\left(w_{n}\right) w_{n}\right) w_{n}=\varphi_{\epsilon}\left(w_{n}\right)\left\|w_{n}\right\|_{\epsilon}^{2}-\int_{\mathbb{R}^{N}} f\left(\varphi_{\epsilon}\left(w_{n}\right) w_{n}\right) w_{n} d x
$$

o que implica

$$
\int_{\mathbb{R}^{N}} \frac{f\left(\varphi_{\epsilon}\left(w_{n}\right) w_{n}\right)}{\varphi_{\epsilon}\left(w_{n}\right)} w_{n} d x=1, \quad \forall n \in \mathbb{N}
$$


Por outro lado, por $\left(f_{2}\right)$ e $\left(f_{3}\right)$, dado $\eta>0$, existe $A_{\eta}>0$ tal que $|f(s)| \leq \eta|s|+A_{\eta}|s|^{p}$, para todo $s \in \mathbb{R}$. Logo,

$$
\begin{aligned}
\int_{\mathbb{R}^{N}} \frac{f\left(\varphi_{\epsilon}\left(w_{n}\right) w_{n}\right)}{\varphi_{\epsilon}\left(w_{n}\right)} w_{n} d x & \leq \int_{\mathbb{R}^{N}}\left(\eta\left|w_{n}\right|^{2}+A_{\eta} \varphi_{\epsilon}\left(w_{n}\right)^{p-1}\left|w_{n}\right|^{p+1}\right) d x \\
& \leq \eta\left\|w_{n}\right\|_{\epsilon}^{2}+C A_{\eta} \varphi_{\epsilon}\left(w_{n}\right)^{p-1}\left\|w_{n}\right\|_{\epsilon}^{p+1} \\
& =\eta+C A_{\eta} \varphi\left(w_{n}\right)^{p-1}
\end{aligned}
$$

Consequentemente,

$$
\lim _{n \rightarrow \infty} \int_{\mathbb{R}^{N}} \frac{f\left(\varphi_{\epsilon}\left(w_{n}\right) w_{n}\right)}{\varphi_{\epsilon}\left(w_{n}\right)} w_{n} d x=0
$$

o que contradiz (1.8). Logo, existe $\delta>0$ tal que $\varphi_{\epsilon}(w) \geq \delta$, para todo $w \in S_{1}(0)$.

Para provar a última sentença deste lema, suponha que exista $\mathcal{A} \subset S_{1}(0)$ compacto e $\left(w_{n}\right) \subset \mathcal{A}$ tal que $\varphi_{\epsilon}\left(w_{n}\right) \rightarrow \infty$. Assim, por (1.7), temos

$$
\int_{\mathbb{R}^{N}} \frac{F\left(\varphi_{\epsilon}\left(w_{n}\right) w_{n}\right)}{\varphi_{\epsilon}\left(w_{n}\right)^{2}} d x \rightarrow \infty
$$

quando $n \rightarrow \infty$. Como $I_{\epsilon}$ é contínua e $\mathcal{A}$ é compacto, $\left|I_{\epsilon}(w)\right| \leq C$, para todo $w \in \mathcal{A}$. Logo

$$
\lim _{n \rightarrow \infty} \frac{I_{\epsilon}\left(w_{n}\right)}{\varphi_{\epsilon}\left(w_{n}\right)^{2}}=0
$$

Porém,

$$
\begin{aligned}
\frac{I_{\epsilon}\left(w_{n}\right)}{\varphi_{\epsilon}\left(w_{n}\right)^{2}} & \leq \max _{s>0} \frac{I_{\epsilon}\left(s w_{n}\right)}{\varphi_{\epsilon}\left(w_{n}\right)^{2}}=\frac{I_{\epsilon}\left(\varphi_{\epsilon}\left(w_{n}\right) w_{n}\right)}{\varphi_{\epsilon}\left(w_{n}\right)^{2}} \\
& =\frac{1}{2}-\int_{\mathbb{R}^{N}} \frac{F\left(\varphi_{\epsilon}\left(w_{n}\right) w_{n}\right)}{\varphi_{\epsilon}\left(w_{n}\right)^{2}} d x \rightarrow-\infty, \quad \text { quando } n \rightarrow \infty,
\end{aligned}
$$

o que nos leva a uma contradição.

Os argumentos das demonstrações do último lema, bem como da próxima proposição, são inspirados no trabalho de Szulkin e Weth em [33].

Proposição 1.2.6. A função $u \mapsto \varphi_{\epsilon}(u)$ é contínua de $E_{\epsilon} \backslash\{0\}$ em $\mathbb{R}_{+}$.

Demonstração. De fato, seja $w_{n} \rightarrow w$ em $E_{\epsilon} \backslash\{0\}$. Uma vez que $\varphi_{\epsilon}(u)=\varphi_{\epsilon}(t u)$ para todo $t>0$, podemos supor sem perda de generalidade que $\left(w_{n}\right) \subset S_{1}(0)$, bem como $w$. Considerando o compacto $\mathcal{A}=\left(w_{n}\right)_{n \in \mathbb{N}} \cup\{w\}$ e aplicando o Lema 1.2.5, temos que existe $\delta>0$ e $C>0$ tais que

$$
\delta \leq \varphi_{\epsilon}\left(w_{n}\right) \leq C, \quad \forall n \in \mathbb{N} .
$$

Logo existe uma subsequência de $\left(w_{n}\right)$ (que continuaremos a denotar por $\left(w_{n}\right)$ ), tal que $\varphi_{\epsilon}\left(w_{n}\right) \rightarrow \varphi>0$. Como $\mathcal{N}_{\epsilon}$ é fechada

$$
\varphi_{\epsilon}\left(w_{n}\right) w_{n} \rightarrow \varphi w \in \mathcal{N}_{\epsilon} .
$$

Assim $\varphi=\varphi_{\epsilon}(w)$ e o resultado segue. 
Primeiramente, observe que por $\left(f_{2}\right)$ e $\left(f_{3}\right)$, segue que existe $\delta>0$ tal que $\|u\|_{\epsilon} \geq \delta$, para todo $u \in \mathcal{N}_{\epsilon}$. Assim, pelo resultado anterior, a aplicação $\mathcal{H}: S_{1}(0) \rightarrow \mathcal{N}_{\epsilon}$ dada por $\mathcal{H}(u)=\varphi_{\epsilon}(u) u$ é contínua. Esta aplicação é também obviamente injetiva e ainda $\mathcal{H}^{-1}(u)=u /\|u\|_{\epsilon}$, que também é contínua. Portanto, $\mathcal{H}$ é um homeomorfismo entre $S_{1}(0)$ e $\mathcal{N}_{\epsilon}$.

Podemos então, tal como Rabinowitz em [30], caracterizar $c_{\epsilon}$ da seguinte forma.

\section{Proposição 1.2.7.}

$$
c_{\epsilon}=c_{\epsilon}^{*}=\inf _{\mathcal{N}_{\epsilon}} I_{\epsilon},
$$

onde

$$
c_{\epsilon}^{*}:=\inf _{u \in E_{\epsilon} \backslash\{0\}} \max _{t \geq 0} I_{\epsilon}(t u) .
$$

Demonstração. Pela construção de $\mathcal{N}_{\epsilon}$, segue que

$$
c_{\epsilon}^{*}=\inf _{u \in E_{\epsilon} \backslash\{0\}} \max _{t \geq 0} I_{\epsilon}(t u)=\inf _{\mathcal{N}_{\epsilon}} I_{\epsilon},
$$

pois $\max _{t \geq 0} I_{\epsilon}(t u)=I_{\epsilon}\left(\varphi_{\epsilon}(u) u\right)$ e $\varphi_{\epsilon}(u) u \in \mathcal{N}_{\epsilon}$, para todo $u \in E_{\epsilon} \backslash\{0\}$.

Seja $g \in \Gamma_{\epsilon}$ e mostremos que $g([0,1]) \cap \mathcal{N}_{\epsilon} \neq \emptyset$. De fato, note primeiramente que $\mathcal{N}_{\epsilon}$, por ser homeomorfo a esfera unitária em $E_{\epsilon}$, divide $E_{\epsilon}$ em duas componentes conexas, uma interna e outra externa a $\mathcal{N}_{\epsilon}$. Se $u \in E_{\epsilon} \backslash\{0\}$ pertence à componente conexa interna a $\mathcal{N}_{\epsilon}$, ou mesmo pertence a $\mathcal{N}_{\epsilon}$, analisando a função $\psi_{\epsilon}(t)=I_{\epsilon}(t u)$, vemos que $\psi_{\epsilon}^{\prime}(t) \geq 0$ para $t \leq \varphi_{\epsilon}(u)$ e que $\varphi_{\epsilon}(u) \geq 1$. Logo

$$
0 \leq \psi_{\epsilon}^{\prime}(1)=I_{\epsilon}^{\prime}(u) u=\|u\|_{\epsilon}^{2}-\int_{\mathbb{R}^{N}} f(u) u d x
$$

o que implicaria

$$
\|u\|_{\epsilon}^{2} \geq \int_{\mathbb{R}^{N}} f(u) u d x .
$$

Assim, pela Observação 1.1.1,

$$
\begin{aligned}
I_{\epsilon}(u) & =\frac{1}{2}\|u\|_{\epsilon}^{2}-\int_{\mathbb{R}^{N}} F(u) d x \\
& \geq \frac{1}{2} \int_{\mathbb{R}^{N}} f(u) u d x-\int_{\mathbb{R}^{N}} F(u) d x \\
& \geq 0
\end{aligned}
$$

e então $g([0,1]) \cap \mathcal{N}_{\epsilon} \neq \emptyset$. Consequentemente,

$$
\max _{t \in[0,1]} I_{\epsilon}(g(t)) \geq \inf _{\mathcal{N}_{\epsilon}} I_{\epsilon}=c_{\epsilon}^{*}
$$

e assim

$$
c_{\epsilon} \geq c_{\epsilon}^{*} .
$$


Por outro lado, para cada $u \in E_{\epsilon} \backslash\{0\}, I_{\epsilon}\left(t_{0} u\right)<0$ para $t_{0}$ grande o suficiente. Assim, o caminho $t u, 0 \leq t \leq t_{0}$ é associado a um $g_{u} \in \Gamma_{\epsilon}$ (depois de um reescalonamento). Dessa forma

$$
\begin{aligned}
c_{\epsilon}^{*} & =\inf _{u \in E_{\epsilon} \backslash\{0\}} \max _{t \geq 0} I_{\epsilon}(t u) \\
& =\inf _{u \in E_{\epsilon} \backslash\{0\}} \max _{t \geq 0} I_{\epsilon}\left(g_{u}(t) u\right) \\
& \geq \inf _{g \in \Gamma_{\epsilon}} \max _{t \geq 0} I_{\epsilon}(t u) \\
& =c_{\epsilon} .
\end{aligned}
$$

Portanto

$$
c_{\epsilon}^{*}=c_{\epsilon}=\inf _{\mathcal{N}_{\epsilon}} I_{\epsilon}
$$

Observação 1.2.8. Observe que como todo ponto crítico não trivial de $I_{\epsilon}$ pertence a $\mathcal{N}_{\epsilon} \mathrm{e}$ $c_{\epsilon}=\inf _{\mathcal{N}_{\epsilon}} I_{\epsilon}$, então se $c_{\epsilon}$ for valor crítico, associado a ele existirá uma solução que terá a menor energia dentre todas as outras não-triviais.

O lema que segue se faz nacessário uma vez que a falta da condição de AmbrosettiRabinowitz em $f$, representa uma dificuldade para se mostrar que as sequências de PalaisSmale para $I_{\epsilon}$ são limitadas. Porém, como veremos, a condição $\left(f_{4}\right)$ será suficiente.

Lema 1.2.9. Seja $\left(v_{n}\right)$ uma sequência $(P S)_{c}$ para $I_{\epsilon}$. Então $\left(v_{n}\right)$ é limitada em $E_{\epsilon}$.

Demonstração. Suponhamos por contradição que $\left\|v_{n}\right\|_{\epsilon} \rightarrow \infty$, quando $n \rightarrow \infty$. Definamos então

$$
w_{n}=\frac{v_{n}}{\left\|v_{n}\right\|_{\epsilon}} .
$$

Sendo $\left(w_{n}\right)$ uma sequência limitada em $E_{\epsilon}$, sabemos que existe $w \in E_{\epsilon}$ tal que, a menos de subsequência, $w_{n} \rightarrow w$ em $E_{\epsilon}$ e, consequentemente, $w_{n} \rightarrow w$ em $L_{l o c}^{q}\left(\mathbb{R}^{N}\right), 1<q<2_{*}$ e também em quase todo ponto de $\mathbb{R}^{N}$, quando $n \rightarrow \infty$.

Afirmamos que $w=0$. De fato, seja

$$
A=\left\{x \in \mathbb{R}^{N} ; w(x) \neq 0\right\}
$$

Devemos mostrar que $A$ tem medida nula. Suponha por contradição que $|A|>0$. Como $I_{\epsilon}\left(v_{n}\right)=c+o_{n}(1)$, dividindo $I_{\epsilon}\left(v_{n}\right)$ por $\left\|v_{n}\right\|_{\epsilon}^{2}$, obtemos

$$
\frac{1}{2}+O_{n}(1)=\int_{\mathbb{R}^{N}} \frac{F\left(v_{n}\right)}{\left\|v_{n}\right\|_{\epsilon}^{2}} d x
$$

Por $\left(f_{1}\right)-\left(f_{5}\right)$ e pela Observação 1.2.13, vemos que

$$
\lim _{s \rightarrow 0} \frac{F(s)}{s^{2}}=0 \quad \text { e } \quad \frac{d}{d s} \frac{F(s)}{s^{2}}=\frac{H(s)}{s^{3}}>0(<0) \text { para } s>0(s<0) .
$$


Logo, $F(s) \geq 0$ para todo $s \in \mathbb{R}$. De (1.9), segue que

$$
\frac{1}{2}+O_{n}(1) \geq \int_{A} \frac{F\left(v_{n}\right)}{\left\|v_{n}\right\|_{\epsilon}^{2}} d x=\int_{A} \frac{F\left(v_{n}\right)}{v_{n}^{2}} w_{n}^{2} d x .
$$

Por $\left(f_{4}\right)$, dado $M>0$ existe $r>0$ tal que

$$
\frac{F(s)}{s^{2}} \geq M \text { para todo }|s|>r .
$$

Combinando (1.10) e (1.11), resulta

$$
O_{n}(1)+\frac{1}{2} \geq \int_{A \cap\left\{\left|v_{n}\right|>r\right\}} \frac{F\left(v_{n}\right)}{v_{n}^{2}} w_{n}^{2} d x \geq M \int_{A \cap\left\{\left|v_{n}\right|>r\right\}} w_{n}^{2} d x .
$$

Usando o Lema de Fatou e observando que $v_{n}(x) \rightarrow+\infty$, quando $n \rightarrow+\infty$, para todo $x \in A$, temos

$$
\frac{1}{2} \geq M \liminf _{n} \int_{A \cap\left\{\left|v_{n}\right|>r\right\}} w_{n}^{2} d x=M \int_{A} w^{2} d x,
$$

o que é impossível porque $\int_{A} w^{2} d x>0$ e $M>0$ é arbritário. Assim, $|A|=0$ e, portanto, $w=0$ em quase todo ponto de $\mathbb{R}^{N}$.

Pelo lema de Lions (ver [23]), uma entre as duas possibilidades ocorre

i) $w_{n} \rightarrow 0$ em $L^{r}\left(\mathbb{R}^{N}\right)$ para $2<r<2_{*}$.

ii) Existe sequência $\left(y_{n}\right) \subset \mathbb{R}^{N}$ e constantes $R, \beta>0$ tais que

$$
\liminf _{n \rightarrow \infty} \int_{B_{R}\left(y_{n}\right)} w_{n}^{2} d x \geq \beta
$$

De fato, suponha que ii) não ocorre, ou seja, que para todo $R>0$,

$$
\sup _{y \in \mathbb{R}^{N}} \int_{B_{R}(y)} w_{n}^{2} d x \rightarrow 0, \quad \text { quando } n \rightarrow \infty .
$$

Observe que $\left(w_{n}\right)$ é limitada em $L^{2}\left(\mathbb{R}^{N}\right)$, enquanto que $\left(\nabla w_{n}\right)$ é limitada em $L^{2^{*}}\left(\mathbb{R}^{N}\right)$, onde $2^{*}=2 N /(N-2)$. Então pelo Lema I.1 de [23], temos que $w_{n} \rightarrow 0$ em $L^{r}\left(\mathbb{R}^{N}\right)$, para $2<r<N 2^{*} /\left(N-2^{*}\right)=2_{*}$, que é exatamente $\left.i\right)$.

Suponhamos então que $i$ ) ocorre. Então por $\left(f_{2}\right),\left(f_{3}\right)$ e por $\left.i\right)$, para todo $\mu>0$,

$$
\lim _{n \rightarrow \infty} \int_{\mathbb{R}^{N}} F\left(\mu w_{n}\right) d x=0 .
$$

Seja $s_{n} \in[0,1]$ tal que

$$
I_{\epsilon}\left(s_{n} v_{n}\right)=\max _{t \in[0,1]} I_{\epsilon}\left(t v_{n}\right) .
$$

Para todo $n$ suficientemente grande,

$$
I_{\epsilon}\left(s_{n} v_{n}\right) \geq I_{\epsilon}\left(\frac{\mu}{\left\|v_{n}\right\|_{\epsilon}} v_{n}\right)=\frac{\mu^{2}}{2}-\int_{\mathbb{R}^{N}} F\left(\mu w_{n}\right) d x .
$$


Por (1.12) e (1.13),

$$
\liminf _{n \rightarrow \infty} I_{\epsilon}\left(s_{n} v_{n}\right) \geq \frac{\mu^{2}}{2}, \quad \text { para todo } \mu>0
$$

Assim

$$
\liminf _{n \rightarrow \infty} I_{\epsilon}\left(s_{n} v_{n}\right)=+\infty .
$$

Como $I_{\epsilon}(0)=0$ e $I_{\epsilon}\left(v_{n}\right) \rightarrow c$, quando $n \rightarrow \infty$, então segue que $s_{n} \in(0,1)$ para todo $n$ suficientemente grande. Portanto, $I_{\epsilon}^{\prime}\left(s_{n} v_{n}\right) s_{n} v_{n}=0$.

Assim, usando a Observação 1.1.1, para todo $t \in[0,1]$ temos que

$$
\begin{aligned}
2 I_{\epsilon}\left(t v_{n}\right) & \leq 2 I_{\epsilon}\left(s_{n} v_{n}\right)-I_{\epsilon}^{\prime}\left(s_{n} v_{n}\right) s_{n} v_{n} \\
& =\int_{\mathbb{R}^{N}}\left(f\left(s_{n} v_{n}\right) s_{n} v_{n}-2 F\left(s_{n} v_{n}\right)\right) d x \\
& \leq \int_{\mathbb{R}^{N}}\left(f\left(v_{n}\right) v_{n}-2 F\left(v_{n}\right)\right) d x \\
& =2 I_{\epsilon}\left(v_{n}\right)+o_{n}(1) \leq C_{1}+o_{n}(1) .
\end{aligned}
$$

Note que, dado $R_{0}>0$ qualquer, para todo $n$ suficientemente grande,

$$
\frac{R_{0}}{\left\|v_{n}\right\|_{\epsilon}}<1
$$

Assim, pela estimativa acima

$$
2 I\left(R_{0} w_{n}\right)=2 I_{\epsilon}\left(\frac{R_{0}}{\left\|v_{n}\right\|} v_{n}\right) \leq C_{1}+o_{n}(1) .
$$

Por outro lado, para todo $R_{0}>0$,

$$
2 I_{\epsilon}\left(R_{0} w_{n}\right)=R_{0}^{2}-2 \int_{\mathbb{R}^{N}} F\left(R_{0} w_{n}\right) d x=R_{0}^{2}-o_{n}(1)
$$

onde usamos i), juntamente com o Teorema da Convergência Dominada. Mas então (1.16) e (1.15) nos fornecem uma contradição, o que significa que $i$ ) não vale.

Assim concluimos que ii) ocorre. Definamos então $\bar{w}_{n}(x):=w_{n}\left(x+y_{n}\right)$. Observe que tal como $\left(w_{n}\right),\left(\bar{w}_{n}\right)$ é uma sequência limitada em $H^{2}\left(\mathbb{R}^{N}\right)$. Assim, existe $\bar{w} \in H^{2}\left(\mathbb{R}^{N}\right)$ tal que a menos de subsequência, $\bar{w}_{n} \rightarrow \bar{w}$ em $H^{2}\left(\mathbb{R}^{N}\right)$. Note ainda que por $\left.i i\right),\|\bar{w}\|_{L^{2}\left(B_{R}(0)\right)}>0$, o que significa que $\bar{w} \neq 0$ em $H^{2}\left(\mathbb{R}^{N}\right)$ e ainda que $\bar{w}(x) \neq 0$ para todo $x$ em um subconunto $\Sigma$ de medida positiva de $B_{R}(0)$.

Uma vez que $\left(I_{\epsilon}\left(v_{n}\right)\right)$ é uma sequência limitada, então

$$
\frac{1}{2}+O_{n}(1)=\int_{\mathbb{R}^{N}} \frac{F\left(v_{n}\right)}{\left\|v_{n}\right\|_{\epsilon}^{2}} d x=\int_{\mathbb{R}^{N}} \frac{F\left(v_{n}\right)}{v_{n}^{2}} w_{n}^{2} d x .
$$

Como $F \geq 0$ em $\mathbb{R}$, temos então que

$$
\begin{aligned}
\frac{1}{2}+O_{n}(1) & =\int_{\mathbb{R}^{N}} \frac{F\left(v_{n}\right)}{v_{n}^{2}} w_{n}^{2} d x \\
& \geq \int_{B_{R}\left(y_{n}\right)} \frac{F\left(v_{n}\right)}{v_{n}^{2}} w_{n}^{2} d x \\
& =\int_{B_{R}(0)} \frac{F\left(v_{n}\left(x+y_{n}\right)\right.}{v_{n}\left(x+y_{n}\right)^{2}} \bar{w}_{n}^{2} d x
\end{aligned}
$$


Observe agora que $v_{n}\left(x+y_{n}\right)=\left\|v_{n}\right\|_{\epsilon}^{2} \bar{w}_{n}(x) \rightarrow+\infty$ para quase todo $x \in \Sigma$. Assim, por $\left(f_{4}\right)$ e pelo Lema de Fatou, segue que

$$
\liminf _{n \rightarrow \infty} \int_{B_{R}(0)} \frac{F\left(v_{n}\left(x+y_{n}\right)\right)}{v_{n}\left(x+y_{n}\right)} \bar{w}_{n}(x) d x=+\infty,
$$

o que contraria (1.17). Dessa forma o item ii) também não vale e essa contradição nos leva ao resultado.

Os argumentos utilizados no começo da demonstração do Lema 1.2.9, justificam a seguinte observação.

Observação 1.2.10. Sob as mesmas hipóteses do Lema 1.2.9, temos que se $\left(v_{n}\right)$ é uma sequência (PS) para $I_{\epsilon}$ e $\left\|v_{n}\right\|_{H^{2}\left(\mathbb{R}^{N}\right)} \rightarrow \infty$, quando $n \rightarrow \infty$, então

$$
w_{n}:=\frac{v_{n}}{\left\|v_{n}\right\|_{\epsilon}} \rightarrow 0, \quad \text { quando } n \rightarrow \infty, \text { em } E_{\epsilon} .
$$

O resultado seguinte auxiliará na prova da existência de solução para o problema (1.3).

Proposição 1.2.11. Considere o seguinte problema

$$
\left\{\begin{aligned}
\Delta^{2} v+\alpha v= & f(v) e m \mathbb{R}^{N} \\
& v \in H^{2}\left(\mathbb{R}^{N}\right) .
\end{aligned}\right.
$$

Se $\alpha>0$ e $f$ satisfaz $\left(f_{1}\right)-\left(f_{5}\right)$, então

$$
c_{\alpha}=\inf _{g \in \Gamma_{\alpha}} \sup _{0 \leq t \leq 1} I_{\alpha}(g(t))>0
$$

é um valor crítico para $I_{\alpha}$, onde $I_{\alpha}$ é o funcional energia associado a (1.18) e $\Gamma_{\alpha}$ é a correspondente classe de caminhos considerada na definição do nivel do passo da montanha.

Demonstração. Seja $E_{\alpha}=\left\{H^{2}\left(\mathbb{R}^{N}\right),\|\cdot\|_{\alpha}\right\}$, onde $\|u\|_{\alpha}^{2}=\int_{\mathbb{R}^{N}}\left(|\Delta u|^{2}+\alpha u^{2}\right) d x$.

Usando os mesmos argumentos da demonstração do Lema 1.2.3, pode-se mostrar que $I_{\alpha}$ satisfaz as condições geométricas do Teorema do Passo da Montanha. Assim, existe uma sequência $\left(w_{n}\right) \subset E_{\alpha}$, tal que

$$
I_{\alpha}\left(w_{n}\right) \rightarrow c_{\alpha} \quad \text { e } \quad I_{\alpha}^{\prime}\left(w_{n}\right) \rightarrow 0, \quad \text { quando } n \rightarrow \infty .
$$

Usando uma argumentação semelhante a do Lema 1.2.9, segue que $\left(w_{n}\right)$ é uma sequência limitada em $E_{\alpha}$.

Afirmamos que existem $\left(y_{n}\right) \subset \mathbb{R}^{N}$ e constantes $R, \beta>0$ tais que

$$
\liminf _{n \rightarrow \infty} \int_{B_{R}\left(y_{n}\right)} w_{n}^{2} d x>\beta .
$$


De fato, caso contrário, para qualquer $R>0$,

$$
\liminf _{n \rightarrow \infty} \sup _{y \in \mathbb{R}^{N}} \int_{B_{R}(y)} w_{n}^{2} d x=0
$$

Pelo Lema I.1 de [23] ( $\operatorname{com} q=2$ e $\left.p=\frac{2 N}{N-2}\right), w_{n} \rightarrow 0$, em $L^{r}\left(\mathbb{R}^{N}\right)$, para todo $2<r<2$. Porém, por $\left(f_{3}\right)$, pelas imersões de Sobolev e pelo Teorema da Convergência Dominada Generalizado,

$$
I_{\alpha}\left(w_{n}\right)-\frac{1}{2} I_{\alpha}^{\prime}\left(w_{n}\right) w_{n}=\int_{\mathbb{R}^{N}}\left(\frac{1}{2} f\left(w_{n}\right) w_{n}-F\left(w_{n}\right)\right) d x \rightarrow 0, \text { quando } n \rightarrow \infty
$$

o que contraria o fato de $I_{\alpha}\left(w_{n}\right)-\frac{1}{2} I_{\alpha}^{\prime}\left(w_{n}\right) w_{n} \rightarrow c_{\alpha}>0$, quando $n \rightarrow \infty$.

Como $I_{\alpha}$ é invariante por translação, então $I_{\alpha}(u)=I_{\alpha}\left(\tau_{y}(u)\right)$, para todo $y \in \mathbb{R}^{N}$, onde $\tau_{y} u(x)=u(x-y)$. Dessa forma, definindo $u_{n}=w_{n} \circ \tau_{y_{n}}$, segue que $\left(u_{n}\right)$ é limitada em $E_{\alpha}$, $I_{\alpha}\left(u_{n}\right) \rightarrow c_{\alpha}, I_{\alpha}^{\prime}\left(u_{n}\right) \rightarrow 0$ e ainda,

$$
\liminf _{n \rightarrow \infty} \int_{B_{R}(0)} u_{n}^{2} d x>\beta
$$

Logo $u_{n}$ converge fracamente para $u \in E_{\alpha} \backslash\{0\}$ e fortemente em $L_{l o c}^{r}\left(\mathbb{R}^{N}\right)$, para $2 \leq r<2_{*}$. Assim $u$ é solução fraca não-trivial de (1.18).

Resta apenas mostrar que $I_{\alpha}(u)=c_{\alpha}$.

Pela Proposição 1.2.7, segue que $c_{\alpha} \leq I_{\alpha}(u)$. Para a desigualdade contrária, seja $\rho>0$ qualquer. Pela Observação 1.1.1,

$$
\begin{aligned}
I_{\alpha}\left(u_{n}\right)-\frac{1}{2} I_{\alpha}^{\prime}\left(u_{n}\right) u_{n} & =\int_{\mathbb{R}^{N}}\left(\frac{1}{2} f\left(u_{n}\right) u_{n}-F\left(u_{n}\right)\right) d x \\
& \geq \int_{B_{\rho}(0)}\left(\frac{1}{2} f\left(u_{n}\right) u_{n}-F\left(u_{n}\right)\right) d x .
\end{aligned}
$$

Assim, pelo lema de Fatou,

$$
c_{\alpha} \geq \int_{B_{\rho}(0)}\left(\frac{1}{2} f(u) u-F(u)\right) d x .
$$

Como a desigualdade vale para todo $\rho>0$, segue pelo Teorema da Convergência Dominada que

$$
c_{\alpha} \geq \int_{\mathbb{R}^{N}}\left(\frac{1}{2} f(u) u-F(u)\right) d x=I_{\alpha}(u)
$$

$\operatorname{Logo} I_{\alpha}(u)=c_{\alpha}$

Munidos desses resultados, podemos agora demonstrar o teorema de existência enunciado no início dessa seção.

Proposição 1.2.12. Se $c_{V_{\infty}}$ é o nível minimax associado ao problema (1.18) com $\alpha=V_{\infty}$, então existe $\epsilon_{0}$ tal que

$$
c_{\epsilon}<c_{V_{\infty}}
$$

para todo $\epsilon \in\left(0, \epsilon_{0}\right)$. 
Demonstração. Seja $w$ a solução de (1.18), tal que $I_{V_{\infty}}(w)=c_{V_{\infty}}$. Seja agora $\chi_{R} \in C^{1}\left(\mathbb{R}^{N}, \mathbb{R}\right)$, tal que $0 \leq \chi_{R} \leq 1, \chi_{R}=1$ em $B_{R}(0), \chi_{R}=0$ em $B_{R+2}(0)^{c}$ e $\left|\nabla \chi_{R}\right| \leq 1$ em $B_{R+2}(0) \backslash B_{R}(0)$. Defina $v_{R}(x)=\chi_{R}(x) w(x)$.

Temos que

$$
\begin{aligned}
\gamma_{R}:=\max _{t \geq 0} I_{V_{\infty}}\left(t v_{R}\right) & \geq I_{V_{\infty}}\left(\varphi_{\epsilon}\left(v_{R}\right) v_{R}\right) \\
& =I_{\epsilon}\left(\varphi_{\epsilon}\left(v_{R}\right) v_{R}\right)+\frac{1}{2} \int_{B_{R+2}(0)}\left(V_{\infty}-V_{\epsilon}(x)\right) \varphi_{\epsilon}\left(v_{R}\right)^{2} v_{R}^{2} d x .
\end{aligned}
$$

Pela caracterização de $c_{\epsilon}$ dada na Proposição 1.2.7, temos que

$$
\gamma_{R} \geq c_{\epsilon}+\frac{1}{2} \int_{B_{R+2}(0)}\left(V_{\infty}-V_{\epsilon}(x)\right) \varphi_{\epsilon}\left(v_{R}\right)^{2} v_{R}^{2} d x .
$$

Seja $R_{1}>0$ grande o suficiente de tal forma que, se $R>R_{1}$

$$
\int_{B_{R+2}(0)} v_{R}^{2} d x \geq \frac{1}{2} \int_{\mathbb{R}^{N}} w^{2} d x
$$

e ainda

$$
\int_{B_{R}}\left(|\Delta w|^{2}+\frac{V_{0}}{2} w^{2}\right) d x \geq \frac{1}{2} \int_{\mathbb{R}^{N}}\left(|\Delta w|^{2}+\frac{V_{0}}{2} w^{2}\right) d x .
$$

Como $I_{\epsilon}^{\prime}\left(\varphi_{\epsilon}\left(v_{R}\right) v_{R}\right) \varphi_{\epsilon}\left(v_{R}\right) v_{R}=0$ e para todo $\eta>0$, existe $A_{\eta}>0$ tal que $|f(s)| \leq$ $\eta|s|+A_{\eta}|s|^{p}$, segue que

$$
\begin{aligned}
\varphi_{\epsilon}\left(v_{R}\right)^{2} \int_{\mathbb{R}^{N}}\left(\left|\Delta v_{R}\right|^{2}+V_{\epsilon} v_{R}^{2}\right) d x & =\int_{\mathbb{R}^{N}} f\left(\varphi_{\epsilon}\left(v_{R}\right) v_{R}\right) \varphi_{\epsilon}\left(v_{R}\right) v_{R} d x \\
& \leq \eta \varphi_{\epsilon}\left(v_{R}\right)^{2} \int_{\mathbb{R}^{N}} v_{R}^{2} d x+A_{\eta} \varphi_{\epsilon}\left(v_{R}\right)^{p+1} \int_{\mathbb{R}^{N}}\left|v_{R}\right|^{p+1} d x
\end{aligned}
$$

Uma vez que

$$
\int_{\mathbb{R}^{N}}\left|v_{R}\right|^{p+1} d x \leq \int_{\mathbb{R}^{N}}|w|^{p+1} d x
$$

e também

$$
\int_{\mathbb{R}^{N}}\left(\left|\Delta v_{R}\right|^{2}+\frac{V_{0}}{2} v_{R}^{2}\right) d x \geq \int_{B_{R}}\left(|\Delta w|^{2}+\frac{V_{0}}{2} w^{2}\right) d x
$$

escolhendo $\eta=\frac{V_{0}}{2}$, segue que para $R>R_{1},(1.22)$ implica

$$
\varphi_{\epsilon}\left(v_{R}\right)^{2} \int_{\mathbb{R}^{N}}\left(\left|\Delta v_{R}\right|^{2}+\frac{V_{0}}{2} v^{2}\right) d x \leq A_{\eta} \varphi_{\epsilon}\left(v_{R}\right)^{p+1} \int_{\mathbb{R}^{N}}\left|v_{R}\right|^{p+1} d x .
$$

Isto por sua vez implica

$$
\frac{1}{2} \varphi_{\epsilon}\left(v_{R}\right)^{2} \int_{\mathbb{R}^{N}}\left(|\Delta w|^{2}+\frac{V_{0}}{2} w^{2}\right) d x \leq A_{\eta} \varphi_{\epsilon}\left(v_{R}\right)^{p+1} \int_{\mathbb{R}^{N}}|w|^{p+1} d x
$$

e finalmente temos

$$
\varphi_{\epsilon}\left(v_{R}\right) \geq\left[\frac{\frac{1}{2} \int_{\mathbb{R}^{N}}\left(|\Delta w|^{2}+\frac{V_{0}}{2} w^{2}\right) d x}{A_{\eta} \int_{\mathbb{R}^{N}}|w|^{p+1} d x}\right]^{\frac{1}{p-1}}=: K>0, \quad \forall R>R_{1} .
$$


Mostremos agora que $\gamma_{R}=c_{V_{\infty}}+\psi(R)$, onde $\psi(R) \rightarrow 0$ quando $R \rightarrow \infty$.

Note que $\gamma_{R}=\max _{t \geq 0} I_{V_{\infty}}\left(t v_{R}\right)=I_{V_{\infty}}\left(\varphi_{V_{\infty}}\left(v_{R}\right) v_{R}\right)=c_{V_{\infty}}+I_{V_{\infty}}\left(\varphi_{V_{\infty}}\left(v_{R}\right) v_{R}\right)-I_{V_{\infty}}(w)$. Como $\varphi_{V_{\infty}}: H^{2}\left(\mathbb{R}^{N}\right) \rightarrow \mathbb{R}_{+}$é contínua e $v_{R} \rightarrow w$ em $H^{2}\left(\mathbb{R}^{N}\right)$ quando $R \rightarrow \infty$, segue que $\varphi_{V_{\infty}}\left(v_{R}\right) v_{R} \rightarrow \varphi_{V_{\infty}}(w) w=w$ quando $R \rightarrow \infty$, já que $w$ é solução de (1.18). Portanto

$$
\psi(R)=I_{V_{\infty}}\left(\varphi_{V_{\infty}}\left(v_{R}\right)\right)-I_{V_{\infty}}(w) \rightarrow 0,
$$

quando $R \rightarrow \infty$.

Seja então $R_{2}>0$ grande o suficiente para que

$$
\psi(R)<\frac{1}{8}\left(V_{\infty}-V(0)\right) K^{2} \int_{\mathbb{R}^{N}} w^{2} d x
$$

sempre que $R>R_{2}$.

Pela continuidade de $V_{\infty}-V(\cdot)$ em 0 e pelo fato de $V_{\infty}-V(0)>0$, existe $\delta>0$ tal que, para $|x|<\delta, V_{\infty}-V(x)>\frac{1}{2}\left(V_{\infty}-V(0)\right)$. Assim, se $\epsilon<\frac{\delta}{R+2}$, então $V_{\infty}-V_{\epsilon}(x)=$ $V_{\infty}-V(\epsilon x)>\frac{1}{2}\left(V_{\infty}-V(0)\right)$ para $x \in B_{R+2}(0)$. Logo, se $\epsilon<\frac{\delta}{R+2}$, a desigualdade (1.19) torna-se

$$
\gamma_{R} \geq c_{\epsilon}+\frac{1}{4} \int_{B_{R+2}(0)}\left(V_{\infty}-V(0)\right) \varphi_{\epsilon}\left(v_{R}\right)^{2} v_{R}^{2} d x .
$$

Consequentemente, se $R>R_{0}:=\max \left\{R_{1}, R_{2}\right\}$, segue de (1.23), (1.24), (1.20) e (1.21), que

$$
c_{V_{\infty}}+\frac{1}{8}\left(V_{\infty}-V(0)\right) K^{2} \int_{\mathbb{R}^{N}} w^{2} d x>c_{\epsilon}+\frac{1}{8}\left(V_{\infty}-V(0)\right) K^{2} \int_{\mathbb{R}^{N}} w^{2} d x,
$$

o que implica

$$
c_{\epsilon}<c_{V_{\infty}}
$$

se $\epsilon<\frac{\delta}{R_{0}+2}:=\epsilon_{0}$.

Observação 1.2.13. Note que seria suficiente para a demonstração do Teorema 1.2.1, mostrar que o funcional $I_{\epsilon}$ satisfaz a condição de Palais-Smale até o nível de energia $c_{V_{\infty}}$. De fato, como no resultado anterior mostramos que existe $\epsilon_{0}>0$ tal que $c_{\epsilon}<c_{V_{\infty}}$ para todo $\epsilon \in\left(0, \epsilon_{0}\right)$, seguiria do Teorema do Passo da Montanha a existência de uma solução fraca não-trivial $v_{\epsilon}$ de (1.3).

No resto dessa seção, vamos estabelecer uma série de lemas que culminará no fato de que $I_{\epsilon}$ satisfaz $(P S)_{c}$ para todo $c<c_{V_{\infty}}$. Esses, por sua vez, são meras adaptações para o operador $\Delta^{2}$ dos resultados análogos enunciados e provados por Alves e Figueiredo em [3].

Lema 1.2.14. Se $\epsilon>0$ e $\left(u_{n}\right)$ for uma sequência $(P S)_{c}$ para $I_{\epsilon}$ em $E_{\epsilon}$ tal que $u_{n} \rightarrow u$ em $E_{\epsilon}$, então fazendo $v_{n}:=u_{n}-u$, temos que $\left(v_{n}\right)$ é uma sequência de Palais-Smale para $I_{\epsilon}$ no nivel $c-I(u)$.

Demonstração. $\quad$ - $I_{\epsilon}\left(v_{n}\right) \rightarrow c-I_{\epsilon}(u)$, quando $n \rightarrow \infty$.

De fato, pelo Lema de Brézis-Lieb [16] e pela convergência fraca de $u_{n}$ para $u$, 


$$
\begin{aligned}
I_{\epsilon}\left(v_{n}\right)-I_{\epsilon}\left(u_{n}\right)+I_{\epsilon}(u)= & \frac{1}{2} \int_{\mathbb{R}^{N}}\left(\left|\Delta u_{n}-\Delta u\right|^{2}-\left|\Delta u_{n}\right|^{2}+|\Delta u|^{2}\right. \\
& \left.+V_{\epsilon}(x)\left(\left|u_{n}-u\right|^{2}-u_{n}^{2}+u^{2}\right)\right) d x \\
& -\int_{\mathbb{R}^{N}}\left(F\left(u_{n}-u\right)-F\left(u_{n}\right)+F(u)\right) d x \\
= & \langle u, u\rangle_{\epsilon}-\left\langle u_{n}, u\right\rangle_{\epsilon}+o_{n}(1) \\
= & o_{n}(1) .
\end{aligned}
$$

- $\left\|I_{\epsilon}^{\prime}\left(v_{n}\right)\right\|_{E_{\epsilon}^{*}}=o_{n}(1)$.

Novamente pelo Lema de Brezis-Lieb e pela convergência fraca, temos que, se $\varphi \in E_{\epsilon}$,

$$
\begin{aligned}
I_{\epsilon}^{\prime}\left(v_{n}\right) \varphi-I_{\epsilon}^{\prime}\left(u_{n}\right) \varphi= & \int_{\mathbb{R}^{N}}\left(\left(\Delta u_{n}-\Delta u\right) \Delta \varphi-\Delta u_{n} \Delta \varphi+V_{\epsilon}(x)\left(\left(u_{n}-u\right) \varphi-u_{n} \varphi\right)\right) d x \\
& -\int_{\mathbb{R}^{N}}\left(f\left(u_{n}-u\right) \varphi-f\left(u_{n}\right) \varphi\right) d x \\
= & -\langle u, \varphi\rangle_{\epsilon}+\int_{\mathbb{R}^{N}} f(u) \varphi d x+o_{n}(1) \\
= & I_{\epsilon}^{\prime}(u) \varphi+o_{n}(1)=o_{n}(1) .
\end{aligned}
$$

Lema 1.2.15. Seja $\epsilon>0$ e $\left(v_{n}\right)$ uma sequência $(P S)_{d}$ para $I_{\epsilon}$ em $E_{\epsilon}$. Então se $v_{n} \rightarrow 0$ em $E_{\epsilon}$ e $v_{n} \nrightarrow 0$ em $E_{\epsilon}$, então

$$
c_{V_{\infty}} \leq d
$$

Demonstração. Seja $s_{n}>0$ tal que $s_{n} v_{n} \in \mathcal{N}_{V_{\infty}}$.

Mostremos primeiramente que

$$
\limsup _{n \rightarrow \infty} s_{n} \leq 1 .
$$

Suponhamos por absurdo que exista uma subsequência de $\left(s_{n}\right)$, que por simplicidade continuaremos a denotar por $\left(s_{n}\right)$, e um $\delta>0$ de tal forma que

$$
s_{n} \geq 1+\delta, \quad \forall n \in \mathbb{N} .
$$

Usando os fatos de que $I_{\epsilon}^{\prime}\left(v_{n}\right) v_{n}=o_{n}(1)$ e $I_{V_{\infty}}^{\prime}\left(s_{n} v_{n}\right) s_{n} v_{n}=0$ para todo $n \in \mathbb{N}$, segue que

$$
\int_{\mathbb{R}^{N}}\left(\frac{f\left(s_{n} v_{n}\right) v_{n}^{2}}{s_{n} v_{n}}-\frac{f\left(v_{n}\right) v_{n}^{2}}{v_{n}}\right) d x=\int_{\mathbb{R}^{N}}\left(V_{\infty}-V_{\epsilon}(x)\right) v_{n}^{2} d x+o_{n}(1) .
$$

De $\left(V_{2}\right)$ segue que dado $\eta>0$, existe $R>0$ tal que $V(\epsilon x) \geq V_{\infty}-\eta$, para todo $x \in \mathbb{R}^{N}$ tal que $|x| \geq R \epsilon^{-1}$. Assim,

$$
\int_{\mathbb{R}^{N}}\left(\frac{f\left(s_{n} v_{n}\right) v_{n}^{2}}{s_{n} v_{n}}-\frac{f\left(v_{n}\right) v_{n}^{2}}{v_{n}}\right) d x \leq \int_{B_{R \epsilon^{-1}(0)}}\left(V_{\infty}-V_{\epsilon}(x)\right) v_{n}^{2} d x+\eta \int_{|x| \geq R \epsilon^{-1}} v_{n}^{2} d x+o_{n}(1) .
$$


Pelas imersões contínuas e compactas de Sobolev e como $v_{n} \rightarrow 0$ em $E_{\epsilon}$ e consequentemente, converge fortemente para 0 em $L_{\text {loc }}^{q}\left(\mathbb{R}^{N}\right)$, para todo $q \in\left(1,2_{*}\right)$, segue que

$$
\int_{\mathbb{R}^{N}}\left(\frac{f\left(s_{n} v_{n}\right)}{s_{n} v_{n}}-\frac{f\left(v_{n}\right)}{v_{n}}\right) v_{n}^{2} d x \leq \eta C+o_{n}(1) .
$$

Afirmamos que existem $R_{1}, \beta>0$ e uma sequência $\left(y_{n}\right) \subset \mathbb{R}^{N}$, tais que

$$
\int_{B_{R_{1}}\left(y_{n}\right)} v_{n}^{2} d x \geq \beta
$$

De fato, caso contrário, para qualquer $R_{1}>0$,

$$
\liminf _{n \rightarrow \infty} \sup _{y \in \mathbb{R}^{N}} \int_{B_{R_{1}}(y)} v_{n}^{2} d x=0 .
$$

Pelo Lema I.1 de [23] ( $\operatorname{com} q=2$ e $\left.p=\frac{2 N}{p-2}\right), v_{n} \rightarrow 0$ quando $n \rightarrow \infty$, em $L^{q}\left(\mathbb{R}^{N}\right)$, para todo $2<q<2_{*}$. Analogamente a (1.4), prova-se que para todo $\rho>0$, existe $C_{\rho}>0$ tal que $|f(s) s| \leq \rho|s|^{2}+C_{\rho}|s|^{p+1}$, para todo $s \in \mathbb{R}$. Então, por $\left(f_{3}\right)$ e pelas imersões de Sobolev, segue que

$$
0 \leq\left\|v_{n}\right\|_{\epsilon}^{2}=\int_{\mathbb{R}^{N}} f\left(v_{n}\right)\left(v_{n}\right) d x+o_{n}(1) \leq \rho \int_{\mathbb{R}^{N}}\left|v_{n}\right|^{2} d x+C_{\rho} \int_{\mathbb{R}^{N}}\left|v_{n}\right|^{p+1} d x+o_{n}(1) .
$$

Assim $v_{n} \rightarrow 0$ em $E_{\epsilon}$, o que contraria nossa hipótese.

Seja então $\bar{v}_{n}(x)=v_{n}\left(x+y_{n}\right)$ e observe que por argumentos semelhantes aos empregados no Lema 1.2.9, $\left(\bar{v}_{n}\right)$ é limitada em $E_{\epsilon}$. Assim, a menos de subsequência $\bar{v}_{n} \rightarrow \bar{v}$ em $E_{\epsilon}$ e por (1.28), $\bar{v} \neq 0$ em um subconjunto $\Lambda \subset B_{R_{1}}(0)$ de medida positiva. Usando agora $\left(f_{5}\right)$, o Lema de Fatou, (1.26) e (1.27) segue que

$$
0<\int_{\Lambda}\left(\frac{f((1+\delta) \bar{v})}{(1+\delta) \bar{v}}-\frac{f(\bar{v})}{\bar{v}}\right) \bar{v}^{2} d x \leq \eta C, \quad \forall \eta>0,
$$

o que é um absurdo. Essa contradição mostra que de fato vale (1.25).

Temos então dois casos a considerar:

i) $\lim _{n \rightarrow+\infty} s_{n}=s<1$

ii) $\lim _{n \rightarrow+\infty} s_{n}=1$.

Se ocorrer $i$ ), então existe uma subsequência de $\left(s_{n}\right)$ (ainda denotada dessa forma) tal que $s_{n} \rightarrow s<1$. Podemos supor sem perda de generalidade que $s_{n}<1$ para todo $n \in \mathbb{N}$. Observe então que

$$
\begin{aligned}
c_{V_{\infty}} & \leq I_{V_{\infty}}\left(s_{n} v_{n}\right) \\
& =I_{V_{\infty}}\left(s_{n} v_{n}\right)-\frac{1}{2} I_{V_{\infty}}^{\prime}\left(s_{n} v_{n}\right) s_{n} v_{n} \\
& =\int_{\mathbb{R}^{N}} \frac{1}{2}\left(f\left(s_{n} v_{n}\right) s_{n} v_{n}-2 F\left(s_{n} v_{n}\right)\right) d x
\end{aligned}
$$


Observe agora que pela Observação 1.1.1,

$$
\int_{\mathbb{R}^{N}} \frac{1}{2}\left(f\left(s_{n} v_{n}\right) s_{n} v_{n}-2 F\left(s_{n} v_{n}\right)\right) d x \leq \int_{\mathbb{R}^{N}} \frac{1}{2}\left(f\left(v_{n}\right) v_{n}-2 F\left(v_{n}\right)\right) d x .
$$

Assim,

$$
\begin{aligned}
c_{V_{\infty}} & \leq \int_{\mathbb{R}^{N}} \frac{1}{2}\left(f\left(v_{n}\right) v_{n}-2 F\left(v_{n}\right)\right) d x \\
& =I_{\epsilon}\left(v_{n}\right)-\frac{1}{2} I_{\epsilon}^{\prime}\left(v_{n}\right) v_{n}+o_{n}(1) \\
& =d+o_{n}(1),
\end{aligned}
$$

de onde segue o resultado.

Se ocorrer ii), existe uma subsequência de $\left(s_{n}\right)$ (que continuaremos a denotar da mesma forma), tal que $s_{n} \rightarrow 1$, quando $n \rightarrow \infty$. Neste caso,

$$
d+o_{n}(1)=I_{\epsilon}\left(v_{n}\right)=I_{V_{\infty}}\left(s_{n} v_{n}\right)+I_{\epsilon}\left(v_{n}\right)-I_{V_{\infty}}\left(s_{n} v_{n}\right) .
$$

De forma que

$$
d+o_{n}(1)=I_{\epsilon}\left(v_{n}\right) \geq c_{V_{\infty}}+I_{\epsilon}\left(v_{n}\right)-I_{V_{\infty}}\left(s_{n} v_{n}\right) .
$$

Note então que

$$
\begin{aligned}
I_{\epsilon}\left(v_{n}\right)-I_{V_{\infty}}\left(s_{n} v_{n}\right)= & \int_{\mathbb{R}^{N}} \frac{\left(1-s_{n}^{2}\right)}{2}\left|\Delta v_{n}\right|^{2} d x+\frac{1}{2} \int_{\mathbb{R}^{N}} V_{\epsilon}(x) v_{n}^{2} d x \\
& -\frac{s_{n}^{2}}{2} \int_{\mathbb{R}^{N}} V_{\infty} v_{n}^{2} d x+\int_{\mathbb{R}^{N}}\left(F\left(s_{n} v_{n}\right)-F\left(v_{n}\right)\right) d x .
\end{aligned}
$$

Observe que pela limitação da sequência $\left(v_{n}\right)$ em $E_{\epsilon}$,

$$
\int_{\mathbb{R}^{N}} \frac{\left(1-s_{n}^{2}\right)}{2}\left|\Delta v_{n}\right|^{2} d x=o_{n}(1)
$$

Ainda, para todo $R>0$, pela compacidade das imersões de Sobolev e pela continuidade de $V$, segue que

$$
\frac{1}{2} \int_{\frac{B_{R}(0)}{}} V_{\epsilon}(x) v_{n}^{2} d x=o_{n}(1)
$$

e ainda

$$
\frac{s_{n}^{2}}{2} \int_{\overline{B_{R}(0)}} V_{\infty} v_{n}^{2} d x=o_{n}(1) .
$$

Dessa forma,

$$
\begin{aligned}
I_{\epsilon}\left(v_{n}\right)-I_{V_{\infty}}\left(s_{n} v_{n}\right) \geq & o_{n}(1)+\frac{1}{2} \int_{B_{R}(0)^{c}} V_{\epsilon}(x) v_{n}^{2} d x-\frac{s_{n}^{2}}{2} \int_{B_{R}(0)^{c}} V_{\infty} v_{n}^{2} d x \\
& +\int_{\mathbb{R}^{N}}\left(F\left(s_{n} v_{n}\right)-F\left(v_{n}\right)\right) d x .
\end{aligned}
$$


Por $\left(V_{2}\right)$, dado $\eta>0$, para $R$ suficientemente grande

$$
\begin{aligned}
I_{\epsilon}\left(v_{n}\right)-I_{V_{\infty}}\left(s_{n} v_{n}\right) \geq & o_{n}(1)+\frac{1}{2} \int_{B_{R}(0)^{c}}\left(V_{\infty}-\eta\right) v_{n}^{2} d x-\frac{s_{n}^{2}}{2} \int_{B_{R}(0)^{c}} V_{\infty} v_{n}^{2} d x \\
& +\int_{\mathbb{R}^{N}}\left(F\left(s_{n} v_{n}\right)-F\left(v_{n}\right)\right) d x
\end{aligned}
$$

ou seja

$$
\begin{aligned}
I_{\epsilon}\left(v_{n}\right)-I_{V_{\infty}}\left(s_{n} v_{n}\right) \geq & o_{n}(1)+\frac{\left(1-s_{n}^{2}\right)}{2} \int_{B_{R}(0)^{c}} V_{\infty} v_{n}^{2} d x-\frac{\eta}{2} \int_{B_{R}(0)^{c}} v_{n}^{2} d x \\
& +\int_{\mathbb{R}^{N}}\left(F\left(s_{n} v_{n}\right)-F\left(v_{n}\right)\right) d x .
\end{aligned}
$$

Logo, pelas imersões de Sobolev e mais a limitação de $\left(v_{n}\right)$ segue que

$$
I_{\epsilon}\left(v_{n}\right)-I_{V_{\infty}}\left(s_{n} v_{n}\right) \geq o_{n}(1)-C \eta+\int_{\mathbb{R}^{N}}\left(F\left(s_{n} v_{n}\right)-F\left(v_{n}\right)\right) d x .
$$

Portanto, aplicando a última desigualdade em (1.29), obtemos

$$
d+o_{n}(1) \geq c_{V_{\infty}}-C \eta+o_{n}(1)+\int_{\mathbb{R}^{N}}\left(F\left(s_{n} v_{n}\right)-F\left(v_{n}\right)\right) d x
$$

Supondo por um instante que $\int_{\mathbb{R}^{N}}\left(F\left(s_{n} v_{n}\right)-F\left(v_{n}\right)\right) d x=o_{n}(1)$, segue que

$$
d+o_{n}(1) \geq c_{V_{\infty}}-C \eta+o_{n}(1) .
$$

Passando o limite em $n \rightarrow \infty$ e depois em $\eta \rightarrow 0$, temos finalmente que

$$
d \geq c_{V_{\infty}}
$$

Para finalizar mostremos que $\int_{\mathbb{R}^{N}}\left(F\left(s_{n} v_{n}\right)-F\left(v_{n}\right)\right) d x=o_{n}(1)$.

Pelo Teorema do Valor Médio segue que

$$
\left|F\left(s_{n} v_{n}\right)-F\left(v_{n}\right)\right|=\left|f\left(\theta_{n}\right)\right|\left|s_{n}-1\right|\left|v_{n}\right|,
$$

onde $\theta_{n} \in\left(v_{n}, s_{n} v_{n}\right)$ ou $\theta_{n} \in\left(s_{n} v_{n}, v_{n}\right)$. Temos

$$
\left|f\left(\theta_{n}\right)\right| \leq \eta\left|\theta_{n}\right|+\left|\theta_{n}\right|^{p} \leq \eta|| v_{n}|+| s_{n}|| v_{n}||+|| v_{n}|+| s_{n}\left\|v_{n}\right\|^{p}
$$

o que implica

$$
\left|f\left(\theta_{n}\right)\right| \leq C \eta\left|v_{n}\right|+C\left|v_{n}\right|^{p}
$$

Assim,

$$
\begin{aligned}
\int_{\mathbb{R}^{N}}\left(F\left(s_{n} v_{n}\right)-F\left(v_{n}\right)\right) d x & \leq C\left|s_{n}-1\right| \int_{\mathbb{R}^{N}} v_{n}^{2} d x+C\left|s_{n}-1\right| \int_{\mathbb{R}^{N}} v_{n}^{p+1} d x \\
& =o_{n}(1),
\end{aligned}
$$

o que finaliza a demonstração. 
Como consequência imediata do Lema 1.2.15 segue o seguinte

Corolário 1.2.16. Se $\left(v_{n}\right)$ é uma sequência $(P S)_{d}$ para $I_{\epsilon}$ tal que $v_{n} \rightarrow 0$ e d $<c_{V_{\infty}}$, então $v_{n} \rightarrow 0 \mathrm{em} E_{\epsilon}$.

Finalmente podemos provar o seguinte resultado.

Proposição 1.2.17. O funcional $I_{\epsilon}$ satisfaz a condição $(P S)_{c}$ para todo $c<c_{V_{\infty}}$.

Demonstração. Seja $\left(u_{n}\right)$ uma sequência $(P S)_{c}$ para $I_{\epsilon}$. Pelo Lema 1.2.9, $\left(u_{n}\right)$ é limitada em $E_{\epsilon}$ e portanto $u_{n} \rightarrow u$ em $E_{\epsilon}$. Sendo $v_{n}=u_{n}-u$, segue que $v_{n} \rightarrow 0$ em $E_{\epsilon}$. Pelo Lema 1.2.14, segue que $\left(v_{n}\right)$ é uma sequência $(P S)_{d}$ para $I_{\epsilon}$, onde $d=c-I_{\epsilon}(u)$. Note que, como $u$ é solução fraca de (1.3), temos que

$$
I_{\epsilon}(u)=I_{\epsilon}(u)-\frac{1}{2} I_{\epsilon}^{\prime}(u) u=\int_{\mathbb{R}^{N}} \frac{1}{2}(f(u) u-2 F(u)) d x \geq 0,
$$

devido a Observação 1.1.1. Portanto $d \leq c<c_{V_{\infty}}$ e $v_{n} \rightarrow 0$ em $E_{\epsilon}$ devido ao Corolário 1.2.16. Isto mostra o resultado.

Pela Observação 1.2.13, existe $\epsilon_{0}>0$ tal que (1.3) possui uma solução fraca não-trivial $v_{\epsilon}$, sempre que $\epsilon \in\left(0, \epsilon_{0}\right)$.

\subsection{Concentração}

Nessa seção vamos mostrar que a sequência de soluções $\left(u_{n}\right)$ obtida na seção anterior, apresenta o fenômeno de concentração.

O resultado principal dessa seção é o seguinte teorema.

Teorema 1.3.1. Considere o problema (1.1), onde $f$ satisfaz $\left(f_{1}\right)-\left(f_{5}\right)$ e $V$, satisfaz $\left(V_{1}\right)$ e $\left(V_{2}\right)$. Para toda sequência $\epsilon_{n} \rightarrow 0$, existe uma subsequência que continuaremos a denotar por $\left(\epsilon_{n}\right)$ tal que (1.1) possui solução $u_{n}:=u_{\epsilon_{n}}$, para todo $n \in \mathbb{N}$. Além disso, se $x_{n}$ é um ponto de máximo de $\left|u_{n}\right|$, então

$$
\lim _{n \rightarrow \infty} V\left(x_{n}\right)=V_{0}
$$

Para facilitar a leitura, a demonstração desse resultado será dividida em vários lemas.

Pelo Teorema 1.2.1, para $\epsilon>0$ suficientemente pequeno, existe $v_{\epsilon}$ solução de (1.3). Sendo $c_{\epsilon}$ o nível minimax associado ao problema (1.3) e $c_{V_{0}}$ o nível minimax associado ao problema

$$
\left\{\begin{array}{l}
\Delta^{2} u+V_{0} u \\
u \in H^{2}\left(\mathbb{R}^{N}\right),
\end{array}=f(u) \text { em } \mathbb{R}^{N}\right.
$$

vale o seguinte resultado. 
26 Capítulo 1 - Existência e concentração de soluções: hipótese global sobre o potencial

\section{Lema 1.3.2.}

$$
\lim _{\epsilon \rightarrow 0} c_{\epsilon}=c_{V_{0}}
$$

Demonstração. Pela Proposição 1.2.11, existe solução não-trivial $w$ para o problema (1.31). Seja $\psi \in C_{0}^{\infty}\left(\mathbb{R}^{N}\right)$ tal que $0 \leq \psi \leq 1, \psi \equiv 0$ em $B_{2}(0)^{c}, \psi \equiv 1$ em $B_{1}(0),|\nabla \psi| \leq C$ e $|\Delta \psi| \leq C$ em $\mathbb{R}^{N}$. Seja ainda

$$
w_{\epsilon}(x)=\psi(\epsilon x) w(x) .
$$

Então $w_{\epsilon} \rightarrow w$ em $H^{2}\left(\mathbb{R}^{N}\right)$ e $I_{V_{0}}\left(w_{\epsilon}\right) \rightarrow I_{V_{0}}(w)$, quando $\epsilon \rightarrow 0$, onde $I_{V_{0}}$ denota o funcional energia associado ao problema (1.31).

Seja $\varphi_{\epsilon}\left(w_{\epsilon}\right)$ tal que $\varphi_{\epsilon}\left(w_{\epsilon}\right) w_{\epsilon} \in \mathcal{N}_{\epsilon}$. Suponhamos por um instante que $\varphi_{\epsilon}\left(w_{\epsilon}\right) \rightarrow 1$, quando $\epsilon \rightarrow 0$.

Então, usando o Teorema da Convergência Dominada, temos

$$
\begin{aligned}
c_{\epsilon} & \leq \max _{t \geq 0} I_{\epsilon}\left(t w_{\epsilon}\right)=I_{\epsilon}\left(\varphi_{\epsilon}\left(w_{\epsilon}\right) w_{\epsilon}\right) \\
& =I_{V_{0}}\left(\varphi_{\epsilon}\left(w_{\epsilon}\right) w_{\epsilon}\right)+\frac{1}{2} \int_{\mathbb{R}^{N}} \varphi_{\epsilon}\left(w_{\epsilon}\right)^{2}\left(V_{\epsilon}(\epsilon x)-V_{0}\right) w_{\epsilon}^{2} d x \\
& \rightarrow I_{V_{0}}(w)=c_{V_{0}},
\end{aligned}
$$

quando $\epsilon \rightarrow 0$. Logo

$$
\limsup _{\epsilon \rightarrow 0} c_{\epsilon} \leq c_{V_{0}}
$$

Mas $I_{V_{0}}(v) \leq I_{\epsilon}(v)$, para todo $v \in H^{2}\left(\mathbb{R}^{N}\right)$ e isto implica $c_{V_{0}} \leq c_{\epsilon}$, para todo $\epsilon>0$. Assim

$$
\liminf _{\epsilon \rightarrow \infty} c_{\epsilon} \geq c_{V_{0}}
$$

Portanto

$$
\lim _{\epsilon \rightarrow 0} c_{\epsilon}=c_{V_{0}}
$$

Mostremos agora que de fato $\varphi_{\epsilon}\left(w_{\epsilon}\right) \rightarrow 1$, quando $\epsilon \rightarrow 0$.

Lembre-se que $\left.\frac{d}{d t} I_{\epsilon}\left(t w_{\epsilon}\right)\right|_{\varphi_{\epsilon}\left(w_{\epsilon}\right)}=0$, ou seja $I_{\epsilon}^{\prime}\left(\varphi_{\epsilon}\left(w_{\epsilon}\right) w_{\epsilon}\right) w_{\epsilon}=0$, o que ocorre se, e somente se,

$$
\varphi_{\epsilon}\left(w_{\epsilon}\right) \int_{\mathbb{R} N}\left(\left|\Delta w_{\epsilon}\right|^{2}+V(\epsilon x) w_{\epsilon}^{2}\right) d x=\int_{\mathbb{R}^{N}} f\left(\varphi_{\epsilon}\left(w_{\epsilon}\right) w_{\epsilon}\right) w_{\epsilon} d x
$$

Para mostrar que $\left(\varphi_{\epsilon}\left(w_{\epsilon}\right)\right)$ é limitada superiormente, suponhamos por contradição que exista uma sequência $\epsilon_{n} \rightarrow 0$, tal que $\varphi_{\epsilon_{n}}\left(w_{\epsilon_{n}}\right) \rightarrow+\infty$. Seja agora $A \subset \mathbb{R}^{N}$ tal que $|A|>0$ e $w(x) \neq 0$, para todo $x \in A$. Pela convergência $L^{q}\left(\mathbb{R}^{N}\right)$ de $w_{\epsilon_{n}}$ para $w$, segue que para todo $n$ 
suficientemente grande, $w_{\epsilon_{n}}(x) \neq 0$, para todo $x \in A$. Assim,

$$
\begin{aligned}
\left\|w_{\epsilon_{n}}\right\|_{\epsilon_{n}}^{2} & =\int_{\mathbb{R}^{N}} \frac{f\left(\varphi_{\epsilon_{n}}\left(w_{\epsilon_{n}}\right) w_{\epsilon_{n}}\right) w_{\epsilon_{n}}}{\varphi_{\epsilon_{n}}\left(w_{\epsilon_{n}}\right)} d x \\
& =\int_{\mathbb{R}^{N}} \frac{f\left(\varphi_{\epsilon_{n}}\left(w_{\epsilon_{n}}\right) w_{\epsilon_{n}}\right) \varphi_{\epsilon_{n}}\left(w_{\epsilon_{n}}\right) w_{\epsilon_{n}}}{\varphi_{\epsilon_{n}}\left(w_{\epsilon_{n}}\right)^{2}} d x \\
& \geq \int_{A} \frac{f\left(\varphi_{\epsilon_{n}}\left(w_{\epsilon_{n}}\right) w_{\epsilon_{n}}\right) \varphi_{\epsilon_{n}}\left(w_{\epsilon_{n}}\right) w_{\epsilon_{n}}}{\varphi_{\epsilon_{n}}\left(w_{\epsilon_{n}}\right)^{2}} d x \\
& \geq \int_{A} \frac{2 F\left(\varphi_{\epsilon_{n}}\left(w_{\epsilon_{n}}\right) w_{\epsilon_{n}}\right) w_{\epsilon_{n}}^{2}}{\left(\varphi_{\epsilon_{n}}\left(w_{\epsilon_{n}}\right) w_{\epsilon_{n}}\right)^{2}} d x,
\end{aligned}
$$

para todo $n \in \mathbb{N}$.

Por outro lado, por $\left(f_{4}\right)$, para todo $x \in A$,

$$
\lim _{n \rightarrow \infty} \frac{F\left(\varphi_{\epsilon_{n}}\left(w_{\epsilon_{n}}\right) w_{\epsilon_{n}}\right) w_{\epsilon_{n}}^{2}}{\left(\varphi_{\epsilon_{n}}\left(w_{\epsilon_{n}}\right) w_{\epsilon_{n}}\right)^{2}}=+\infty
$$

Consequentemente, pelo Lema de Fatou,

$$
\liminf _{n \rightarrow \infty} \int_{A} \frac{2 F\left(\varphi_{\epsilon_{n}}\left(w_{\epsilon_{n}}\right) w_{\epsilon_{n}}\right) w_{\epsilon_{n}}^{2}}{\left(\varphi_{\epsilon_{n}}\left(w_{\epsilon_{n}}\right) w_{\epsilon_{n}}\right)^{2}} d x=+\infty
$$

Logo,

$$
\left\|w_{\epsilon_{n}}\right\|_{\epsilon_{n}}^{2} \rightarrow+\infty, \quad \text { quando } n \rightarrow \infty,
$$

o que contraria o fato de $w_{\epsilon_{n}} \rightarrow w$, quando $n \rightarrow \infty$.

Afirmamos agora que $\varphi_{\epsilon}\left(w_{\epsilon}\right) \nrightarrow 0$, quando $\epsilon \rightarrow 0$. De fato, do contrário, existiria uma sequência $\epsilon_{n} \rightarrow 0$ tal que $\varphi_{\epsilon_{n}}\left(w_{\epsilon_{n}}\right) \rightarrow 0$ quando $n \rightarrow \infty$. Por $\left(f_{2}\right)-\left(f_{3}\right)$ e argumentando como na demonstração do Lema 1.2.5, resulta

$$
\lim _{n \rightarrow \infty} \int_{\mathbb{R}^{N}} \frac{f\left(\varphi_{\epsilon_{n}}\left(w_{\epsilon_{n}}\right) w_{\epsilon_{n}}\right) w_{\epsilon_{n}}^{2}}{\varphi_{\epsilon_{n}}\left(w_{\epsilon_{n}}\right) w_{\epsilon_{n}}} d x=0
$$

Por outro lado,

$$
\left\|w_{\epsilon_{n}}\right\|_{\epsilon_{n}}^{2}=\int_{\mathbb{R}^{N}} \frac{f\left(\varphi_{\epsilon_{n}}\left(w_{\epsilon_{n}}\right) w_{\epsilon_{n}}\right) w_{\epsilon_{n}}^{2}}{\varphi_{\epsilon_{n}}\left(w_{\epsilon_{n}}\right) w_{\epsilon_{n}}} d x
$$

Portanto, de (1.32) e (1.33), vemos que $\left\|w_{\epsilon_{n}}\right\|_{\epsilon_{n}} \rightarrow 0$, contradizendo o fato que $w_{\epsilon_{n}} \rightarrow w$ com $I_{V_{0}}(w)=c_{V_{0}}>0$.

Assim, existem $\alpha, \beta>0$ tais que ao longo de uma subsequência,

$$
\alpha \leq \varphi_{\epsilon_{n}}\left(w_{\epsilon_{n}}\right) \leq \beta .
$$

Portanto, toda subsequência convergente de $\varphi_{\epsilon_{n}}\left(w_{\epsilon_{n}}\right)$, converge para 1, pois se $\bar{\varphi}$ é o limite de $\left(\varphi_{\epsilon_{k}}\left(w_{k}\right)\right)$, então

$$
\|w\|_{H^{2}}^{2}=\int_{\mathbb{R}^{N}} \frac{f(\bar{\varphi} w) w}{\bar{\varphi}} d x
$$

e portanto $\bar{\varphi} w \in \mathcal{N}_{V_{0}}$. Mas $w \in \mathcal{N}_{V_{0}}$ e $\log \bar{\varphi}=1$.

Logo, $\varphi_{\epsilon}\left(w_{\epsilon}\right) \rightarrow 1$, pois toda subsequência possui uma subsequência convergente e ainda toda subsequência convergente, converge para 1 . 
Seja $\epsilon_{n} \rightarrow 0$ quando $n \rightarrow \infty$ uma sequência qualquer. Observe que, denotando por $v_{n}:=v_{\epsilon_{n}}$, uma modificação simples do Lema 1.2.9 mostra que a sequência $\left(v_{n}\right)$ é limitada.

Lema 1.3.3. Existe sequência $\left(y_{n}\right) \subset \mathbb{R}^{N}, R, \beta>0$ tais que

$$
\liminf _{n \rightarrow \infty} \int_{B_{R}\left(y_{n}\right)} v_{n}^{2} d x \geq \beta
$$

Demonstração. De fato, caso contrário, para qualquer $R>0$,

$$
\liminf _{n \rightarrow \infty} \sup _{y \in \mathbb{R}^{N}} \int_{B_{R}(y)} v_{n}^{2} d x=0
$$

Pelo Lema I.1 de [23] ( $\operatorname{com} q=2$ e $\left.p=\frac{2 N}{p-2}\right), v_{n} \rightarrow 0$ quando $n \rightarrow \infty$, em $L^{r}\left(\mathbb{R}^{N}\right)$, para todo $2<r<2_{*}$. Porém, por $\left(f_{3}\right)$, pelas imersões de Sobolev e pelo Teorema da Convergência Dominada Generalizado,

$$
I_{\epsilon_{n}}\left(v_{n}\right)-\frac{1}{2} I_{\epsilon_{n}}^{\prime}\left(v_{n}\right) v_{n}=\int_{\mathbb{R}^{N}}\left(\frac{1}{2} f\left(v_{n}\right) v_{n}-F\left(v_{n}\right)\right) d x \rightarrow 0, \text { quando } n \rightarrow \infty
$$

o que contraria o fato de $I_{\epsilon_{n}}\left(v_{n}\right)-\frac{1}{2} I_{\epsilon_{n}}^{\prime}\left(v_{n}\right) v_{n} \rightarrow c_{V_{0}}>0$, quando $n \rightarrow \infty$.

Defina

$$
w_{n}(x)=v_{n}\left(x+y_{n}\right)=u_{n}\left(\epsilon_{n} x+\epsilon_{n} y_{n}\right)
$$

Note que $w_{n}$ satisfaz

$$
\left\{\begin{array}{l}
\Delta^{2} w_{n}+V\left(\epsilon_{n} x+\epsilon_{n} y_{n}\right) w_{n}=f\left(w_{n}\right) \text { em } \mathbb{R}^{N} \\
w \in H^{2}\left(\mathbb{R}^{N}\right)
\end{array}\right.
$$

e ainda

$$
\liminf _{n \rightarrow \infty} \int_{B_{R}(0)} w_{n}^{2} d x \geq \beta
$$

Lema 1.3.4. $\left(\epsilon_{n} y_{n}\right)$ é limitado quando $n \rightarrow \infty$.

Demonstração. De fato, suponha por absurdo que exista uma subsequência que ainda denotaremos por $\left(\epsilon_{n} y_{n}\right)$, tal que $\epsilon_{n} y_{n} \rightarrow \infty$. Como $\left(w_{n}\right)$ é limitada, então $w_{n} \rightarrow w_{0}$ em $H^{2}\left(\mathbb{R}^{N}\right)$ e $w_{n} \rightarrow w_{0}$ em $L_{\text {loc }}^{q}\left(\mathbb{R}^{N}\right)$ para $2 \leq q<2_{*}$, quando $n \rightarrow \infty$, onde $w_{0} \neq 0$ por (1.35). Pode-se provar que $w_{0}$ satisfaz o problema limite (1.18) com $\alpha=V_{\infty}$.

Usando então o Lema de Fatou, a Observação 1.1.1, $\left(V_{2}\right)$ e o Lema 1.3.2, temos que

$$
\begin{aligned}
c_{V_{0}} & <c_{V_{\infty}} \\
& \leq I_{V_{\infty}}\left(w_{0}\right) \\
& =I_{V_{\infty}}\left(w_{0}\right)-\frac{1}{2} I_{V_{\infty}}^{\prime}\left(w_{0}\right) w_{0} \\
& =\int_{\mathbb{R}^{N}}\left(\frac{1}{2} f\left(w_{0}\right) w_{0}-F\left(w_{0}\right)\right) d x \\
& \leq \liminf _{n \rightarrow \infty} \int_{\mathbb{R}^{N}}\left(\frac{1}{2} f\left(w_{n}\right) w_{n}-F\left(w_{n}\right)\right) d x \\
& =\liminf _{n \rightarrow \infty} c_{\epsilon_{n}}=c_{V_{0}},
\end{aligned}
$$

o que é uma contradição. 
Observe que no resultado anterior, $c_{V_{0}}<c_{V_{\infty}}$ pois o nível minimax $c_{\alpha}$ associado ao problema (1.18), é estritamente crescente em $\alpha>0$.

Pelo resultado anterior, podemos supor sem perda de generalidade, que existe $x_{0} \in \mathbb{R}^{N}$ tal que $\epsilon_{n} y_{n} \rightarrow x_{0}$ quando $n \rightarrow \infty$ e ainda que $w_{n} \rightarrow w_{0}$ em $H^{2}\left(\mathbb{R}^{N}\right)$, com $w_{0} \neq 0$.

Lema 1.3.5. $x_{0}$ é um mínimo global de $V$.

Demonstração. Por (1.34) e aplicando teoria de regularidade, pode-se provar que na verdade $w_{n} \rightarrow w_{0}$ em $C_{\text {loc }}^{4}\left(\mathbb{R}^{N}\right)$, quando $n \rightarrow \infty$. Assim, para cada $x \in \mathbb{R}^{N}$, fazendo $n \rightarrow \infty$, temos que

$$
\Delta^{2} w_{0}(x)+V\left(x_{0}\right) w_{0}(x)=f\left(w_{0}(x)\right) .
$$

Dessa forma,

$$
\int_{\mathbb{R}^{N}}\left(\left|\Delta w_{0}\right|^{2}+V_{0} w_{0}^{2}\right) d x \leq \int_{\mathbb{R}^{N}}\left(\left|\Delta w_{0}\right|^{2}+V\left(x_{0}\right) w_{0}^{2}\right) d x=\int_{\mathbb{R}^{N}} f\left(w_{0}\right) w_{0} d x
$$

de modo que existe $0<\tau \leq 1$ tal que $\tau w_{0} \in \mathcal{N}_{V_{0}}$.

Então, pela Observação 1.1.1 e pelo Lema de Fatou,

$$
\begin{aligned}
c_{V_{0}} & =\lim _{n \rightarrow \infty} c_{\epsilon_{n}} \\
& =\liminf _{n \rightarrow \infty} \int_{\mathbb{R}^{N}}\left(\frac{1}{2} f\left(w_{n}\right) w_{n} d x-F\left(w_{n}\right)\right) d x \\
& \geq \int_{\mathbb{R}^{N}}\left(\frac{1}{2} f\left(w_{0}\right) w_{0} d x-F\left(w_{0}\right)\right) d x \\
& \geq \int_{\mathbb{R}^{N}}\left(\frac{1}{2} f\left(\tau w_{0}\right) \tau w_{0} d x-F\left(\tau w_{0}\right)\right) d x \\
& =I_{V_{0}}\left(\tau w_{0}\right) \geq c_{V_{0}} .
\end{aligned}
$$

$\operatorname{Logo} \tau=1$ e $w_{0} \in \mathcal{N}_{V_{0}}$, ou seja

$$
\int_{\mathbb{R}^{N}}\left(\left|\Delta w_{0}\right|^{2}+V\left(x_{0}\right) w_{0}^{2}\right) d x=\int_{\mathbb{R}^{N}} f\left(w_{0}\right) w_{0} d x=\int_{\mathbb{R}^{N}}\left(\left|\Delta w_{0}\right|^{2}+V_{0} w_{0}^{2}\right) d x,
$$

o que implica $V\left(x_{0}\right)=V_{0}$, pois $w_{0} \neq 0$.

Lema 1.3.6. $w_{n} \rightarrow w_{0}$ em $H^{2}\left(\mathbb{R}^{N}\right)$, quando $n \rightarrow \infty$.

Demonstração. Lembre-se que $I_{\epsilon_{n}}\left(v_{n}\right)=c_{\epsilon_{n}} \rightarrow c_{V_{0}}$, quando $n \rightarrow \infty$. Para cada $n \in \mathbb{N}$, seja $\varphi_{0}\left(w_{n}\right)>0$ tal que $\tilde{w}_{n}=\varphi_{0}\left(w_{n}\right) w_{n} \in \mathcal{N}_{V_{0}}$.

Mostremos que $I_{V_{0}}\left(\tilde{w}_{n}\right) \rightarrow c_{V_{0}}$. 


$$
\begin{aligned}
c_{V_{0}} & \leq I_{V_{0}}\left(\tilde{w}_{n}\right) \\
& =\frac{1}{2} \int_{\mathbb{R}^{N}}\left(\left|\Delta \tilde{w}_{n}\right|^{2}+V_{0} \tilde{w}_{n}^{2}\right) d x-\int_{\mathbb{R}^{N}} F\left(\tilde{w}_{n}\right) d x \\
& \leq \frac{1}{2} \int_{\mathbb{R}^{N}}\left(\left|\Delta \tilde{w}_{n}\right|^{2}+V\left(\epsilon_{n} x+\epsilon_{n} y_{n}\right) \tilde{w}_{n}^{2}\right) d x-\int_{\mathbb{R}^{N}} F\left(\tilde{w}_{n}\right) d x \\
& =\frac{1}{2} \int_{\mathbb{R}^{N}}\left(\left|\Delta\left(\varphi_{0}\left(w_{n}\right) v_{n}\right)\right|^{2}+V\left(\epsilon_{n} x\right)\left(\varphi_{0}\left(w_{n}\right) v_{n}\right)^{2}\right) d x-\int_{\mathbb{R}^{N}} F\left(\varphi_{0}\left(w_{n}\right) v_{n}\right) d x \\
& =I_{\epsilon_{n}}\left(\varphi_{0}\left(w_{n}\right) v_{n}\right) \\
& \leq I_{\epsilon_{n}}\left(v_{n}\right) \\
& =c_{\epsilon_{n}}=c_{V_{0}}+o_{n}(1)
\end{aligned}
$$

e isto implica $I_{V_{0}}\left(\tilde{w}_{n}\right) \rightarrow c_{V_{0}}$ quando $n \rightarrow \infty$.

Mostremos agora que $\varphi_{0}\left(w_{n}\right) \rightarrow \varphi_{0}>0$, a menos de subsequência.

Primeiramente, existe $M>0$ tal que a menos de subsequência, $\left|\varphi_{0}\left(w_{n}\right)\right| \leq M$, para todo $n \in \mathbb{N}$. De fato, como $w_{n} \nrightarrow 0$, segue que existe $\delta>0$ tal que $\left\|w_{n}\right\|_{H^{2}\left(\mathbb{R}^{N}\right)}>\delta$, para todo $n \in \mathbb{N}$.

Como, $I_{V_{0}}\left(\tilde{w}_{n}\right) \rightarrow c_{V_{0}}$, e $\left(\tilde{w}_{n}\right) \subset \mathcal{N}_{V_{0}}$, então é fácil mostrar que $\left(\tilde{w}_{n}\right)$ é limitada em $H^{2}\left(\mathbb{R}^{N}\right)$. Assim,

$$
\left|\varphi_{0}\left(w_{n}\right)\right| \delta<\left|\varphi_{0}\left(w_{n}\right)\right|\left\|w_{n}\right\|_{H^{2}\left(\mathbb{R}^{N}\right)} \leq\left\|\varphi_{0}\left(w_{n}\right) w_{n}\right\|_{H^{2}\left(\mathbb{R}^{N}\right)} \leq K,
$$

e isto implica

$$
\left|\varphi_{0}\left(w_{n}\right)\right| \leq \frac{K}{\delta}=M
$$

para todo $n \in \mathbb{N}$.

Dessa forma, a menos de subsequência, $\varphi_{0}\left(w_{n}\right) \rightarrow \varphi_{0} \geq 0$.

Afirmamos ainda que $\varphi_{0}>0$, pois caso contrário,

$$
\left\|\tilde{w}_{n}\right\|_{H^{2}\left(\mathbb{R}^{N}\right)}=\left\|\varphi_{0}\left(w_{n}\right) w_{n}\right\|_{H^{2}\left(\mathbb{R}^{N}\right)}=\left|\varphi_{0}\left(w_{n}\right)\right|\left\|w_{n}\right\|_{H^{2}\left(\mathbb{R}^{N}\right)} \rightarrow 0
$$

quando $n \rightarrow \infty$, o que é um absurdo.

Portanto, $\tilde{w}_{n}=\varphi_{0}\left(w_{n}\right) w_{n} \rightarrow \varphi_{0} w_{0} \neq 0$ em $H^{2}\left(\mathbb{R}^{N}\right)$.

O resultado segue agora do próximo Lema, aplicado a $\left(\tilde{w}_{n}\right)$.

Lema 1.3.7. Seja $\left(z_{n}\right) \subset H^{2}\left(\mathbb{R}^{N}\right)$ tal que $I_{V_{0}}\left(z_{n}\right) \rightarrow c_{V_{0}}$ e $z_{n} \in \mathcal{N}_{V_{0}}$, para todo $n \in \mathbb{N}$. Se $z_{n} \rightarrow z \neq 0$, então $z_{n} \rightarrow z$ a menos de subsequência.

Demonstração. Pelo Princípio Variacional de Ekeland, podemos supor sem perda de generalidade que $\left(z_{n}\right)$ é uma sequência $(P S)_{c_{V_{0}}}$ para $I_{V_{0}}$ em $H^{2}\left(\mathbb{R}^{N}\right)$. Como

$$
\begin{array}{cc}
z_{n} \rightarrow z & \text { em } H^{2}\left(\mathbb{R}^{N}\right), \\
z_{n} \rightarrow z & \text { em } L_{\text {loc }}^{q}\left(\mathbb{R}^{N}\right), \text { para } 2 \leq q<2_{*}, \\
z_{n} \rightarrow z & \text { em quase todo ponto de } \mathbb{R}^{N},
\end{array}
$$


pelo fato de ser $f$ subcrítica e pelas imersões compactas de Sobolev, segue que $I_{V_{0}}^{\prime}(z)=0$. Isto é, $z \in \mathcal{N}_{V_{0}}$.

Note ainda que, pela Observação 1.1.1 e pelo Lema de Fatou,

$$
\begin{aligned}
c_{V_{0}} & =\lim _{n \rightarrow \infty} I_{V_{0}}\left(z_{n}\right) \\
& =\lim _{n \rightarrow \infty}\left[\int_{\mathbb{R}^{N}}\left(\frac{1}{2} f\left(z_{n}\right) z_{n}-F\left(z_{n}\right)\right) d x+o_{n}(1)\right] \\
& \geq \int_{\mathbb{R}^{N}}\left(\frac{1}{2} f(z) z-F(z)\right) d x \\
& =I_{V_{0}}(z) \\
& \geq c_{V_{0}},
\end{aligned}
$$

ou seja

$$
I_{V_{0}}(z)=c_{V_{0}} .
$$

Seja $v_{n}=z_{n}-z$ e observe que pelo Lema de Brézis-Lieb, $I_{V_{0}}\left(v_{n}\right) \rightarrow c_{V_{0}}-I_{V_{0}}(z)=0$ e $\left\|I_{V_{0}}^{\prime}\left(v_{n}\right)\right\|_{H^{-2}\left(\mathbb{R}^{N}\right)} \rightarrow 0$, quando $n \rightarrow \infty$.

Note então que $v_{n} \rightarrow 0 \mathrm{em} H^{2}\left(\mathbb{R}^{N}\right)$ e queremos mostrar que de fato $v_{n} \rightarrow 0$ em $H^{2}\left(\mathbb{R}^{N}\right)$, quando $n \rightarrow \infty$. Para isso, suponhamos por absurdo que $v_{n} \nrightarrow 0$ em $H^{2}\left(\mathbb{R}^{N}\right)$. Usando argumentos semelhantes aos do Lema 1.2.15, vamos mostrar que $\left(v_{n}\right)$ é uma sequência $(P S)_{d}$ para $I_{V_{0}} \operatorname{com} d \geq c_{V_{0}}$, o que gerará uma contradição com o fato de ser $d=0<c_{V_{0}}$.

Seja $s_{n}>0$ tal que $s_{n} v_{n} \in \mathcal{N}_{V_{0}}$. Mostremos primeiramente que

$$
\limsup _{n \rightarrow \infty} s_{n} \leq 1 .
$$

Suponhamos por absurdo que exista uma subsequência de $\left(s_{n}\right)$, que por simplicidade continuaremos a denotar por $\left(s_{n}\right)$, e um $\delta>0$ de tal forma que

$$
s_{n} \geq 1+\delta, \quad \forall n \in \mathbb{N} .
$$

Usando argumentos semelhantes aos do Lema 1.2.9, segue que $\left(v_{n}\right)$ é uma sequência limitada em $H^{2}\left(\mathbb{R}^{N}\right)$. Então, como $I_{V_{0}}^{\prime}\left(v_{n}\right) v_{n}=o_{n}(1)$ e $I_{V_{0}}^{\prime}\left(s_{n} v_{n}\right) s_{n} v_{n}=0$ para todo $n \in \mathbb{N}$, segue que

$$
\int_{\mathbb{R}^{N}}\left(\frac{f\left(s_{n} v_{n}\right)}{s_{n} v_{n}}-\frac{f\left(v_{n}\right)}{v_{n}}\right) v_{n}^{2} d x=o_{n}(1) .
$$

Assim como no Lema 1.2.15, pelo Lema de Lions [23], existem $R, \beta>0$ e uma sequência $\left(y_{n}\right) \subset \mathbb{R}^{N}$, tais que

$$
\liminf _{n \rightarrow \infty} \int_{B_{R}\left(y_{n}\right)} v_{n}^{2} d x \geq \beta .
$$

Seja então $\bar{v}_{n}(x)=v_{n}\left(x+y_{n}\right)$ e observe que por argumentos semelhantes aos empregados no Lema 1.2.9, $\left(\bar{v}_{n}\right)$ é limitada em $H^{2}\left(\mathbb{R}^{N}\right)$. Assim, a menos de subsequência $\bar{v}_{n} \rightarrow \bar{v}$ em $H^{2}\left(\mathbb{R}^{N}\right)$ e por $(1.40), \bar{v} \neq 0$ em um subconjunto $\Lambda \subset B_{R_{1}}(0)$ de medida positiva. Usando agora $\left(f_{5}\right)$, o Lema de Fatou, (1.38) e (1.39) segue que

$$
0<\int_{\Lambda}\left(\frac{f((1+\delta) \bar{v})}{(1+\delta) \bar{v}}-\frac{f(\bar{v})}{v_{n}}\right) \bar{v}^{2} d x=0
$$


o que é um absurdo. Essa contradição mostra que de fato vale (1.37).

Temos então dois casos a considerar:

- Existe uma subsequência de $\left(s_{n}\right)$ (ainda denotada desta forma) tal que $s_{n} \rightarrow s<1$. Podemos supor sem perda de generalidade que $s_{n}<1$ para todo $n \in \mathbb{N}$. Observe então que pela Observação 1.1.1

$$
\begin{aligned}
c_{V_{0}} & \leq I_{V_{0}}\left(s_{n} v_{n}\right) \\
& =I_{V_{0}}\left(s_{n} v_{n}\right)-\frac{1}{2} I_{V_{0}}^{\prime}\left(s_{n} v_{n}\right) s_{n} v_{n} \\
& =\int_{\mathbb{R}^{N}}\left(\frac{1}{2} f\left(s_{n} v_{n}\right) s_{n} v_{n}-F\left(s_{n} v_{n}\right)\right) d x \\
& \leq \int_{\mathbb{R}^{N}}\left(\frac{1}{2} f\left(v_{n}\right) v_{n}-F\left(v_{n}\right)\right) d x \\
& =I_{V_{0}}\left(v_{n}\right)-\frac{1}{2} I_{0}^{\prime}\left(v_{n}\right) v_{n}+o_{n}(1) \\
& =d+o_{n}(1)
\end{aligned}
$$

de onde segue o resultado.

- Existe uma subsequência de $\left(s_{n}\right)$ (que continuaremos a denotar da mesma forma), tal que $s_{n} \rightarrow 1$, quando $n \rightarrow \infty$. Neste caso,

$$
d+o_{n}(1)=I_{V_{0}}\left(v_{n}\right)=I_{V_{0}}\left(s_{n} v_{n}\right)+I_{V_{0}}\left(v_{n}\right)-I_{V_{0}}\left(s_{n} v_{n}\right) .
$$

De forma que

$$
d+o_{n}(1) \geq c_{V_{0}}+I_{V_{0}}\left(v_{n}\right)-I_{V_{0}}\left(s_{n} v_{n}\right) .
$$

Observe que

$$
\begin{aligned}
I_{V_{0}}\left(v_{n}\right)-I_{V_{0}}\left(s_{n} v_{n}\right) & =\frac{\left(1-s_{n}^{2}\right)}{2} \int_{\mathbb{R}^{N}}\left(\left|\Delta v_{n}\right|^{2} d x+V_{0} v_{n}^{2}\right) d x+\int_{\mathbb{R}^{N}}\left(F\left(s_{n} v_{n}\right)-F\left(v_{n}\right)\right) d x \\
& =o_{n}(1),
\end{aligned}
$$

onde a última igualdade segue de maneira análoga ao Lema 1.2.15.

Portanto, (1.41) torna-se

$$
d+o_{n}(1) \geq c_{V_{0}}+o_{n}(1),
$$

o que implica

$$
d \geq c_{V_{0}}
$$

e isto encerra a demonstração. 
Pelas imersões contínuas de $H^{2}\left(\mathbb{R}^{N}\right)$ em $L^{q}\left(\mathbb{R}^{N}\right)$ para $2 \leq q \leq 2_{*}$, segue que $w_{n} \rightarrow w_{0}$ em $L^{2 *}\left(\mathbb{R}^{N}\right)$. Assim, pela recíproca do Teorema de Frechét-Kolmogorov (critério de compacidade forte em $L^{r}\left(\mathbb{R}^{N}\right)$ ), temos que

$$
\int_{B_{R}^{c}(0)}\left|w_{n}\right|^{2 *} d x \rightarrow 0, \quad \text { quando } R \rightarrow \infty
$$

uniformemente com respeito a $n$.

O próximo resultado prova um decaimento uniforme para a família $\left(w_{n}\right)$ que será essencial na demonstração do fenômeno de concentração para as soluções $u_{n}$.

Lema 1.3.8. $w_{n}(x) \rightarrow 0$, quando $|x| \rightarrow \infty$, uniformemente em $n$.

Demonstração. Vamos admitir por um instante que existe $K>0$, tal que

$$
\left\|w_{n}\right\|_{L^{\infty}\left(\mathbb{R}^{N}\right)} \leq K, \forall n \in \mathbb{N} .
$$

A demonstração desse fato será dada na Seção 1.4 a seguir.

Como $\left(w_{n}\right)$ é limitada em $L^{\infty}\left(\mathbb{R}^{N}\right)$, então dado $x \in \mathbb{R}^{N}$, segue que $w_{n} \in L^{q}\left(B_{1}(x)\right)$, para todo $q \geq 1$. Como $w_{n}$ é solução de (1.34), segue do Teorema 7.1 de [2], que

$$
\begin{aligned}
\left\|w_{n}\right\|_{W^{4, q}\left(B_{1}(x)\right)} & \leq C\left(\left\|f\left(w_{n}\right)\right\|_{L^{q}\left(B_{2}(x)\right)}+\left\|w_{n}\right\|_{L^{q}\left(B_{2}(x)\right)}\right) \\
& \leq C\left\|w_{n}\right\|_{L^{q}\left(B_{2}(x)\right)} \\
& \leq C\left\|w_{n}\right\|_{L^{\infty}\left(\mathbb{R}^{N}\right)}^{\frac{q-2 *}{q}}\left\|w_{n}\right\|_{L^{2 *}\left(B_{2}(x)\right)}^{2^{*}} \\
& =C\left\|w_{n}\right\|_{L^{2 *}\left(B_{2}(x)\right)}^{2 *},
\end{aligned}
$$

onde a constante $C>0$ acima independe de $x$ e de $n$. Observe que na desigualdade acima foi usado a seguinte estimativa

$$
\begin{aligned}
\left\|f\left(w_{n}\right)\right\|_{L^{q}\left(B_{2}(x)\right)}^{q} & =\int_{B_{2}(x)}\left|f\left(w_{n}\right)\right|^{q} d y \\
& \leq C \int_{B_{2}(x)}\left(\left|w_{n}\right|+\left|w_{n}\right|^{p}\right)^{q} d y \\
& \leq C\left[\left\|w_{n}\right\|_{L^{q}\left(B_{2}(x)\right)}+\left(\int_{B_{2}(x)}\left|w_{n}\right|^{p q} d y\right)^{\frac{1}{q}}\right]^{q} \\
& \leq C\left[\left\|w_{n}\right\|_{L^{q}\left(B_{2}(x)\right)}+K\left\|w_{n}\right\|_{L^{q}\left(B_{2}(x)\right)}\right]^{q} \\
& \leq C\left\|w_{n}\right\|_{L^{q}\left(B_{2}(x)\right)} .
\end{aligned}
$$

Para $q>N$, teremos a seguinte imersão contínua $W^{4, q}\left(B_{1}(x)\right) \hookrightarrow C^{3, \alpha}\left(\overline{B_{1}(x)}\right)$, para $\alpha \in\left(0,1-\frac{N}{q}\right)$. Dessa forma,

$$
\begin{aligned}
\left\|w_{n}\right\|_{C^{3, \alpha}\left(\overline{B_{1}(x)}\right)} & \leq C\left\|w_{n}\right\|_{W^{4, q}\left(B_{1}(x)\right)} \\
& \leq C\left\|w_{n}\right\|_{L^{2 *}\left(B_{2}(x)\right)}^{2_{*}} .
\end{aligned}
$$


Usando agora (1.43), vemos que

$$
\left|w_{n}(x)\right| \rightarrow 0, \quad \text { quando }|x| \rightarrow \infty
$$

uniformemente com relação a $n$.

Afirmamos que existe $\rho>0$ tal que $\left\|w_{j}\right\|_{L^{\infty}\left(\mathbb{R}^{N}\right)}>\rho$, para todo $j \in \mathbb{N}$, ao longo de uma subsequência de $\left(w_{n}\right)$. De fato, supondo que $\left\|w_{n}\right\|_{L^{\infty}\left(\mathbb{R}^{N}\right)} \rightarrow 0$, quando $n \rightarrow \infty$, então, para todo $\eta>0$,

$$
\begin{aligned}
\left\|w_{n}\right\|_{H^{2}\left(\mathbb{R}^{N}\right)}^{2} & \leq C \int_{\mathbb{R}^{N}}\left(\left|\Delta w_{n}\right|^{2}+V\left(\epsilon_{n} x+\epsilon_{n} y_{n}\right) w_{n}\right) d x \\
& =C \int_{\mathbb{R}^{N}} f\left(w_{n}\right) w_{n} d x \\
& \leq C\left(\eta\left\|w_{n}\right\|_{L^{2}\left(\mathbb{R}^{N}\right)}^{2}+A_{\eta}\left\|w_{n}\right\|_{L^{p+1}\left(\mathbb{R}^{N}\right)}^{p+1}\right)
\end{aligned}
$$

o que implica, se $\eta<\frac{1}{2}$,

$$
\left\|w_{n}\right\|_{H^{2}\left(\mathbb{R}^{N}\right)}^{2} \leq A_{\eta}\left\|w_{n}\right\|_{L^{\infty}\left(\mathbb{R}^{N}\right)}^{p+1}\left\|w_{n}\right\|_{L^{p}\left(\mathbb{R}^{N}\right)}^{p} \rightarrow 0
$$

quando $n \rightarrow \infty$. Assim $\left\|w_{n}\right\|_{H^{2}\left(\mathbb{R}^{N}\right)}^{2} \rightarrow 0$, quando $n \rightarrow \infty$, o que contradiz o fato de $w_{n} \rightarrow w_{0}$ em $H^{2}\left(\mathbb{R}^{N}\right)$ e $w_{0} \not \equiv 0$.

Seja agora $x_{n}$ um ponto de máximo de $\left|u_{n}\right|$ em $\mathbb{R}^{N}$, então

$$
p_{n}:=\frac{x_{n}-\epsilon_{n} y_{n}}{\epsilon_{n}}
$$

é um ponto de máximo para $\left|w_{n}\right|$. Pelo Lema 1.3.6, existe $R_{0}>0$ tal que $p_{n} \in B_{R_{0}}(0)$ para todo $n$ suficientemente grande. Então, a menos de subsequência, $p_{n} \rightarrow p_{0}$ quando $n \rightarrow \infty$. Assim

$$
x_{n}=\epsilon_{n} p_{n}+\epsilon_{n} y_{n} \rightarrow x_{0}, \text { quando } n \rightarrow \infty .
$$

Portanto a sequência de pontos de máximo $x_{n}$ de $\left|u_{n}\right|$ satisfaz

$$
x_{n} \rightarrow x_{0} \text {, quando } n \rightarrow \infty,
$$

o que prova o Teorema 1.3.1.

\subsection{Estimativa $L^{\infty}$}

Agora nos resta apenas demonstrar a seguinte estimativa, estabelecida por Ramos em [31], que foi suposta verdadeira na seção anterior.

Proposição 1.4.1. $\left(w_{n}\right)_{n \in \mathbb{N}}$ é limitada em $L^{\infty}\left(\mathbb{R}^{N}\right)$.

Para a prova desta proposição, assumiremos os seguintes lemas, cujas demonstrações podem ser encontradas em [31]. 
Lema 1.4.2. Para todo $p \in(1, \infty)$ e $h \in L^{p}\left(\mathbb{R}^{N}\right)$, o problema

$$
\Delta^{2} u+u=h \quad u \in \mathcal{S}^{\prime}\left(\mathbb{R}^{N}\right),
$$

tem uma única solução u. Essa solução é dada por

$$
u=\hat{\beta} * h,
$$

onde $\beta(x)=1 /\left(1+|2 \pi x|^{4}\right)$ e $\hat{\beta}$ é a Transformada de Fourier de $\beta$ Ainda, $u \in W^{4, p}\left(\mathbb{R}^{N}\right) e$ $\|u\|_{W^{4, p}\left(\mathbb{R}^{N}\right)} \leq c(p)\|h\|_{p}$, para alguma constante $c(p)>0$.

Lema 1.4.3. Existe $p_{0} \in\left(2_{*}, \infty\right)$ e um $\delta>0$ tais que, toda função $q_{\delta} \in L^{\frac{N}{4}\left(\mathbb{R}^{N}\right)}$ com $\left\|q_{\delta}\right\|_{\frac{N}{4}} \leq \delta$, induz um operador linear $A: L^{p_{0}}\left(\mathbb{R}^{N}\right) \longrightarrow L^{p_{0}}\left(\mathbb{R}^{N}\right) \cap L^{2_{*}}\left(\mathbb{R}^{N}\right)$, dado por

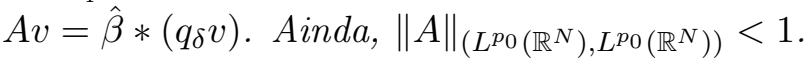

Demonstração. Fazendo $g_{n}\left(x, w_{n}\right)=f\left(w_{n}\right)-V\left(\epsilon_{n} x+\epsilon_{n} y_{n}\right) w_{n}$, temos que $w_{n}$ satisfaz

$$
\Delta^{2} w_{n}=g_{n}\left(x, w_{n}\right) \text { em } \mathbb{R}^{N} .
$$

A nova não-linearidade $g_{n}$ satisfaz também a condição de crescimento subcrítico, ou seja

$$
\left|g_{n}(x, s)\right| \leq C\left(|s|+|s|^{p}\right)=C\left(|s|+\alpha(s)|s|^{2_{*}-1}\right), \forall n \in \mathbb{N},
$$

onde $\alpha(s)=|s|^{p-2_{*}+1} \rightarrow 0$, quando $s \rightarrow \infty$, pois $p<2_{*}-1$.

Denote por $f_{n}(x)=w_{n}(x)+g_{n}\left(x, w_{n}(x)\right)$ e observe que $w_{n}$ satisfaz

$$
\Delta^{2} w_{n}+w_{n}=f_{n} \text { em } \mathcal{S}^{\prime}\left(\mathbb{R}^{N}\right)
$$

Seja $f_{n}^{1}=f_{n} \chi_{\left\{x ;\left|w_{n}(x)\right| \leq 1\right\}}$ e $f_{n}^{2}=f_{n} \chi_{\left\{x ;\left|w_{n}(x)\right|>1\right\}}$ e note que $f_{n}=f_{n}^{1}+f_{n}^{2}$. Sejam agora $w_{n}^{1}$ e $w_{n}^{2}$ tais que

$$
\Delta^{2} w_{n}^{i}+w_{n}^{i}=f_{n}^{i}, \text { onde } i=1,2
$$

no sentido distribucional.

Observe que $f_{n}^{1} \in L_{2_{*}}\left(\mathbb{R}^{N}\right) \cap L^{\infty}\left(\mathbb{R}^{N}\right)$, pois $\left|f_{n}^{1}(x)\right| \leq C$, para todo $n \in \mathbb{N}$. Ainda, pelas Imersões de Sobolev,

$$
\int_{\mathbb{R}^{N}}\left|f_{n}^{1}\right|^{2 *} d x \leq C \int_{\left\{x ;\left|w_{n}\right| \leq 1\right\}}\left|w_{n}\right|^{2 *} d x<K, \forall n \in \mathbb{N} .
$$

Assim, pela Desigualdade de Interpolação, $f_{n}^{1} \in L^{\alpha}\left(\mathbb{R}^{N}\right)$, para todo $\alpha \in\left[2_{*}, \infty\right]$, com $\left\|f_{n}^{1}\right\|_{\alpha} \leq K(\alpha)$, para todo $n \in \mathbb{N}$. Escolhendo $\alpha>2 *$ grande de forma que $4 \alpha>N$, teremos a imersão $W^{4, \alpha}\left(\mathbb{R}^{N}\right) \hookrightarrow C^{0}\left(\mathbb{R}^{N}\right)$. Assim, pelo Lema 1.4.2, $f_{n}^{1} \in L^{\alpha}\left(\mathbb{R}^{N}\right)$ implica $\left\|w_{n}^{1}\right\|_{W^{4, \alpha}\left(\mathbb{R}^{N}\right)} \leq C(\alpha)\left\|f_{n}^{1}\right\|_{\alpha} \leq C^{\prime}(\alpha)$, para todo $n \in \mathbb{N}$. Assim

$$
\left\|w_{n}^{1}\right\|_{C^{0}(\mathbb{R} N)} \leq C^{\prime}(\alpha), \forall n \in \mathbb{N} .
$$


Ainda, $f_{n}^{2} \in L^{\frac{2 *}{2 *-1}}\left(\mathbb{R}^{N}\right)$.

Pelo Lema 1.4.2, $w_{n}^{i}$ existem e são únicas. Note ainda que $w_{n}=w_{n}^{1}+w_{n}^{2}$. Ainda, pelo mesmo motivo que $w_{n}, w_{n}^{i}$ satisfazem a equação (1.44) em $\mathcal{S}^{\prime}\left(\mathbb{R}^{N}\right)$.

Seja $a_{n}(x)$ tal que $f_{n}^{2}(x)=a_{n}(x) w_{n}(x)$, ou seja,

$$
a_{n}(x)= \begin{cases}1+\frac{g_{n}\left(x, w_{n}(x)\right)}{w_{n}(x)} & \text { se }\left|w_{n}(x)\right|>1 \\ 0 & \text { se }\left|w_{n}(x)\right| \leq 1 .\end{cases}
$$

Observe que, dado $j \in \mathbb{N}$, denotando por $\Omega_{j}=\left\{x \in \mathbb{R}^{N} ;|u(x)| \leq n \mathrm{e}|u(x)|^{2_{*}-2} \leq n\right\}$, temos que

$$
\left\|a_{n}\right\|_{L^{\frac{N}{4}\left(\mathbb{R}^{N} \backslash \Omega_{j}\right)}} \leq C \alpha\left(\left|w_{n}\right|\right)\left\|w_{n}\right\|_{L^{2 *}\left(\mathbb{R}^{N} \backslash \Omega_{j}\right)}, \forall n \in \mathbb{N},
$$

pois para $\left|w_{n}(x)\right|>1$,

$$
\begin{aligned}
\left|a_{n}(x)\right| & \leq 1+\frac{\left|g_{n}\left(x, w_{n}\right)\right|}{\left|w_{n}\right|} \\
& \leq C+C\left|w_{n}(x)\right|^{p-1} \\
& =C+C\left|w_{n}\right|^{\frac{p-1}{2_{*}-2}}\left|w_{n}\right|^{2_{*}-2} \\
& \leq C^{\prime}\left|w_{n}\right|^{\frac{p-1}{2_{*}-2}}\left|w_{n}\right|^{2_{*}-2} \\
& =C^{\prime} \alpha\left(\left|w_{n}\right|\right) w_{n}^{2_{*}-2} .
\end{aligned}
$$

Dessa forma,

$$
\int_{\mathbb{R}^{N} \backslash \Omega_{j}}\left|a_{n}\right|^{\frac{N}{4}} d x \leq \int_{\omega_{j}} C^{\prime \prime} \alpha\left(\left|w_{n}\right|\right)\left|w_{n}\right|^{2_{*}} d x=C^{\prime \prime} \alpha\left(\left|w_{n}\right|\right)^{\frac{N}{4}}\left\|w_{n}\right\|_{L^{2 *}\left(\omega_{j}\right)}^{2_{*}},
$$

onde $\omega_{j}=\left\{x ;\left|w_{n}(x)\right| \geq \min \left\{j, j^{\frac{1}{2 *-2}}\right\}\right\}$.

Seja $\delta$ e $p_{0}$ como no Lema 1.4.3 e seja $j \in \mathbb{N}$ tal que $\alpha(|s|)<\delta^{\frac{N}{4}}, \forall|s|>j$. Assim

$$
\int_{\mathbb{R}^{N} \backslash \Omega_{j}}\left|a_{n}\right|^{\frac{N}{4}} d x \leq C^{\prime} \delta\left\|w_{n}\right\|_{L^{2 *\left(\mathbb{R}^{N}\right)}} \leq \frac{\delta}{2}^{\frac{N}{4}},
$$

para todo $n \in \mathbb{N}$ e para o $j$ fixado.

Fazendo

$$
q_{n}^{\delta}(x)=\left\{\begin{array}{cc}
\frac{a_{n}(x)}{n} & x \in \Omega_{j} \\
a_{n}(x) & x \notin \Omega_{j}
\end{array},\right.
$$

onde $n(\delta)$ é tal que $\frac{1}{n(\delta)} \int_{\Omega_{j}}\left|a_{n}\right|^{\frac{N}{4}} d x<\frac{\delta}{2}{ }^{\frac{N}{4}}, \forall n \in \mathbb{R}^{N}$ e para o $j$ fixado. Assim, para todo $n \in \mathbb{N}$,

$$
\left\|q_{n}^{\delta}\right\|_{L^{\frac{N}{4}\left(\mathbb{R}^{N}\right)}}<\delta .
$$

Seja então $f_{n}^{2}=q_{n}^{\delta} w_{n}+f_{n}^{\delta}$, onde $f_{n}^{\delta}=f_{n}^{2}-q_{n}^{\delta} w_{n}$, ou seja,

$$
f_{n}^{\delta}=\left\{\begin{array}{ll}
\left(1-\frac{1}{n}\right) a_{n}(x) w_{n}(x) & x \in \Omega_{j} \\
0 & x \notin \Omega_{j}
\end{array} .\right.
$$


Note que $f_{n}^{\delta} \in L^{2 *}\left(\mathbb{R}^{N}\right) \cap L^{\infty}\left(\mathbb{R}^{N}\right)$, pois

$$
\int_{\mathbb{R}^{N}}\left|f_{n}^{\delta}\right|^{2 *} d x=\left(1-\frac{1}{n}\right) \int_{\Omega_{j}}\left|a_{n}\right|^{2 *}\left|w_{n}\right|^{2 *} d x \leq C\left\|w_{n}\right\|_{2_{*}}^{2_{*}} \leq C n,
$$

para todo $n \in \mathbb{N}$. Ainda $\left|f_{n}^{\delta}(x)\right| \leq C\left|a_{n}(x)\right|\left|w_{n}(x)\right| \leq C j$, para todo $n \in \mathbb{N}$.

Temos então que $w_{n}$ satisfaz a equação

$$
\Delta^{2} w_{n}+w_{n}=q_{n}^{\delta} w_{+} \bar{f}_{n},
$$

onde $\bar{f}_{n}=f_{n}^{1}+f_{n}^{2}$ e $\bar{f}_{n} \in L^{2 *}\left(\mathbb{R}^{N}\right) \cap L^{\infty}\left(\mathbb{R}^{N}\right)$ com limitação uniforme em $n$.

Pela Lema 1.4.2, existe $h_{n} \in W^{4, \alpha}\left(\mathbb{R}^{N}\right), \alpha \in\left[2_{*}, \infty\right]$ tal que

$$
\Delta^{2} h_{n}+h_{n}=\bar{f}_{n} \mathrm{em} \mathcal{S}^{\prime}\left(\mathbb{R}^{N}\right) \text {. }
$$

Seja $p_{0} \in\left(2_{*}, \infty\right)$ tal como no Lema 1.4.3. Como $\left\|q_{n}^{\delta}\right\|_{\frac{N}{4}} \leq \delta$, temos que, para cada $n$, está bem definido o operador $A_{n}$ tal como no Lema 1.4.3.

Como $\left\|A_{n}\right\|_{\mathcal{L}\left(L^{p_{0}}\right)}<1,\left(I-A_{n}\right)$ é inversível, para todo $n \in \mathbb{N}$. Seja então $v_{n} \in L^{p_{0}}\left(\mathbb{R}^{N}\right)$, tal que $v_{n}-A_{n} v_{n}=h_{n}$. Obsesrve que

$$
v_{n}=h_{n}+A_{n} v_{n} \in L^{2 *}\left(\mathbb{R}^{N}\right) \cap L^{p_{0}}\left(\mathbb{R}^{N}\right.
$$

e ainda que $v_{n}$ satisfaz a equação

$$
\Delta^{2} v_{n}+v_{n}=\bar{f}_{n}
$$

pois a solução de

$$
\Delta^{2} z+z=q_{n}^{\delta} v_{n}+\bar{f}_{n} \mathrm{em} \mathcal{S}^{\prime}\left(\mathbb{R}^{N}\right)
$$

é dada por $\hat{\beta} *\left(q_{n} v_{n}+\bar{f}_{n}\right)=A_{n} v_{n}+h_{n}=v_{n}$.

Dessa forma,

$$
\Delta^{2}\left(v_{n}-w_{n}\right)+\left(v_{n}-w_{n}\right)=q_{n}^{\delta}\left(v_{n}-w_{n}\right) \operatorname{em} \mathcal{S}^{\prime}\left(\mathbb{R}^{N}\right),
$$

ou seja, $A_{n}\left(v_{n}-w_{n}\right)=v_{n}-w_{n}$ e como $\|A\|_{\mathcal{L}\left(L^{2 *}\right)}<1$, segue que $v_{n}=w_{n}$.

Então $w_{n} \in L^{p_{0}\left(\mathbb{R}^{N}\right)} \mathrm{e}$

$$
\begin{aligned}
\left\|w_{n}\right\|_{p_{0}} & \leq\left\|h_{n}\right\|_{p_{0}}+\left\|A_{n} v_{n}\right\|_{p_{0}} \\
& \leq\left\|h_{n}\right\|_{p_{0}}+\left\|A_{n}\right\|_{\mathcal{L}\left(L^{2 *}\right)}\left\|w_{n}\right\|_{p_{0}} .
\end{aligned}
$$

Isto implica

$$
\begin{aligned}
C\left\|w_{n}\right\|_{p_{0}} & \leq\left\|h_{n}\right\|_{p_{0}} \\
& \leq C\left\|\bar{f}_{n}\right\|_{p_{0}} \\
& \leq C\left(\left\|f_{n}^{1}\right\|_{p_{0}}+\left\|f_{n}^{\delta}\right\|_{p_{0}}\right) \\
& \leq C^{\prime}\left\|f_{n}^{1}\right\|_{2_{*}}^{\frac{2_{*}}{p_{0}}}+C^{\prime \prime}\left\|f_{n}^{\delta}\right\|_{2_{*}}^{\frac{2_{*}}{p_{0}}} \leq \bar{C}
\end{aligned}
$$


38 Capítulo 1 - Existência e concentração de soluções: hipótese global sobre o potencial

para todo $n \in \mathbb{N}$.

Em resumo $w_{n}=w_{n}^{1}+w_{n}^{2}$, onde $w_{n}^{1} \in L^{\alpha}\left(\mathbb{R}^{N}\right), \forall \alpha \in\left[2_{*}, \infty\right]$ e $\left\|w_{n}^{2}\right\|_{\alpha} \leq C(\alpha)$, para todo $n \in \mathbb{N}$.

Ainda, $w_{n}^{2} \in L^{p_{0}}\left(\mathbb{R}^{N}\right)$, para algum $p_{0}>2_{*}, \operatorname{com}\left\|w_{n}^{2}\right\|_{p^{0}} \leq C, \forall n \in \mathbb{N}$.

Como

$$
\begin{aligned}
\left|f_{n}^{2}(x)\right| & \leq\left|w_{n}(x)+\right| g_{n}\left(x, w_{n}(x)\right) \mid \\
& \leq C\left(\left.\left|w_{n}(x)+\right| w_{n}(x)\right|^{p-1}\right) \\
& \leq C\left|w_{n}(x)\right|^{2 *-1}
\end{aligned}
$$

para todo $x \in \mathbb{R}^{N}$, podemos aplicar um argumento do tipo bootstrap em

$$
\Delta^{2} w_{n}^{2}+w_{n}^{2}=f_{n}^{2} \mathrm{em} \mathcal{S}^{\prime}\left(\mathbb{R}^{N}\right)
$$

para concluir que, para cada $i \in \mathbb{N}$, existe um $K(i)>0$, tal que

$$
\left\|w_{n}\right\|_{p_{i}} \leq K(i), \forall n \in \mathbb{N}
$$

onde $p_{i}=\frac{N p_{i}}{N\left(2_{*}-1\right)-4 p_{i}}$.

Como $p_{0}>2_{*}, p_{i} \rightarrow \infty$ e assim, existe $i_{0}$ tal que $\frac{2_{*}-1}{p_{i_{0}}}-\frac{4}{N} \leq 0$, de forma que

$$
W^{4, \frac{p_{i}}{2 *-1}}\left(\mathbb{R}^{N}\right) \hookrightarrow L^{s}\left(\mathbb{R}^{N}\right), \forall s \in[1, \infty) .
$$

Logo $w_{n} \in L^{s}\left(\mathbb{R}^{N}\right)$.

Portanto $w_{n} \in W^{4, \frac{s}{2 *-1}}\left(\mathbb{R}^{N}\right)$.

Fixando $s>N\left(2_{*}-1\right)$, teremos $W^{4, \frac{s}{2_{*}-1}}\left(\mathbb{R}^{N}\right) \hookrightarrow C^{1, \alpha}\left(\mathbb{R}^{N}\right)$ e assim $\left\|w_{n}\right\|_{\infty}$ é limitado uniformemente em $n$. 


$-\frac{10}{2}$

\section{Existência e concentração de soluções: hipótese local sobre o potencial}

\subsection{Introdução}

Nesse capítulo, vamos estudar questões sobre a existência de soluções que apresentam fenômeno de concentração, para o seguinte problema

$$
\left\{\begin{array}{r}
\epsilon^{4} \Delta^{2} u+V(x) u=f(u) \text { em } \mathbb{R}^{N} \\
u \in H^{2}\left(\mathbb{R}^{N}\right),
\end{array}\right.
$$

onde $\epsilon>0, N \geq 5$ e o potencial $V$, ao contrário que no capítulo anterior, satisfaz uma hipótese local. A não-linearidade $f$ é sublinear na origem e possui um crescimento subcrítico no infinito. Da mesma forma como no Capítulo 1, vamos admitir que $f$ satisfaz uma condição de superlinearidade mais fraca que a condição de Ambrosetti-Rabinowitz. Mais especificamente, vamos supor que $f$ satisfaça as mesmas hipóteses dadas no Capítulo 1 :

$\left(f_{1}\right) f \in C^{1}(\mathbb{R})$,

$\left(f_{2}\right) f(0)=f^{\prime}(0)=0$, 
$\left(f_{3}\right)$ existem constantes $c_{1}, c_{2}>0$ e $p \in\left(1,2_{*}-1\right)$, tais que $|f(s)| \leq c_{1}|s|+c_{2}|s|^{p}$, para todo $s \in \mathbb{R}$, onde $2_{*}=\frac{2 N}{N-4}$,

$\left(f_{4}\right) \lim _{|s| \rightarrow \infty} \frac{F(s)}{s^{2}}=+\infty$, onde $F(s)=\int_{0}^{s} f(t) d t$,

$\left(f_{5}\right) \frac{f(s)}{s}$ é crescente para $s>0$ e decrescente para $s<0$.

Quanto à função $V$, vamos supor as seguintes condições:

$\left(V_{1}\right) V \in C^{0}\left(\mathbb{R}^{N}\right) \cap L^{\infty}\left(\mathbb{R}^{N}\right)$,

$\left(V_{3}\right)$ existe $\Omega \subset \mathbb{R}^{N}$ limitado com interior não vazio e $x_{0} \in \Omega$, tal que

$$
0<V_{0}=\inf _{\mathbb{R}^{N}} V<\inf _{\partial \Omega} V
$$

Desejamos provar o seguinte resultado

Teorema 2.1.1. Sejam $V$ e $f$ satisfazendo $\left(V_{1}\right)$ e $\left(V_{3}\right)$ e $\left(f_{1}\right)-\left(f_{5}\right)$, respectivamente. Então para toda sequência $\epsilon_{n} \rightarrow 0$, existe uma subsequência que continuaremos a denotar por $\left(\epsilon_{n}\right)$ tal que (2.1) (com $\epsilon_{n}$ no lugar de $\epsilon$ ) possui uma solução não-trivial $u_{n} \in H^{2}\left(\mathbb{R}^{N}\right)$. Ainda mais, sendo $x_{n}$ ponto de máximo de $\left|u_{n}\right|$, então $x_{n} \in \Omega$ e ainda

$$
\lim _{n \rightarrow \infty} V\left(x_{n}\right)=\inf _{\mathbb{R}^{N}} V
$$

$\mathrm{Na}$ abordagem direta do problema (2.1), algumas dificuldades naturalmente surgem. Uma delas é a dificuldade de se verificar a condição de Palais-Smale. Por isso, para obter existência de solução, seguindo os argumentos de Jeanjean e Tanaka em [22], vamos fazer uma modificação na não-linearidade de forma a recuperar a condição (PS). Posteriormente, mostraremos que a solução do passo da montanha do problema modificado, de fato é solução do problema original.

\subsection{O problema modificado}

Considere $V_{0}$ dado por $\left(V_{1}\right)$. Sejam $k>2 V_{0}$ e $a>0$ tais que $\max \left\{\frac{f(a)}{a}, \frac{f(-a)}{-a}\right\} \leq \frac{V_{0}}{k}$. A existência de tal $a$ segue da continuidade de $f$ e das condições $\left(f_{2}\right)$ e $\left(f_{4}\right)$.

Defina

$$
\tilde{f}(s)=\left\{\begin{array}{lll}
\frac{-f(-a)}{a} s & \text { se } & s<-a \\
f(s) & \text { se } & |s| \leq a \\
\frac{f(a)}{a} s & \text { se } & s>a
\end{array}\right.
$$

Pela continuidade de $V$, existe um aberto $\Omega^{\prime} \subset \Omega$, não-vazio, com fronteira suave e tal que:

$$
\inf _{\Omega \backslash \Omega^{\prime}} V>\inf _{\mathbb{R}^{N}} V \quad \text { e } \quad \min _{\partial \Omega^{\prime}} V>\inf _{\mathbb{R}^{N}} V
$$


Seja agora $\chi \in C^{\infty}\left(\mathbb{R}^{N}\right), 0 \leq \chi \leq 1$, tal que

$$
\chi(x)= \begin{cases}1, & \text { se } x \in \Omega^{\prime} \\ 0, & \text { se } x \in \mathbb{R}^{N} \backslash \Omega,\end{cases}
$$

Por fim, defina $g(x, s)=\chi(x) f(s)+(1-\chi(x)) \tilde{f}(s)$.

Considere o problema

$$
\left\{\begin{array}{r}
\epsilon^{4} \Delta^{2} u+V(x) u=g(x, u) \text { em } \mathbb{R}^{N} \\
u \in H^{2}\left(\mathbb{R}^{N}\right) .
\end{array}\right.
$$

Por $\left(f_{1}\right)$ - $\left(f_{5}\right)$, a função $g$ satisfaz as seguintes condições:

$\left(g_{1}\right) g(x, s)=o(|s|)$, quando $s \rightarrow 0$,

$\left(g_{2}\right)$ existem constantes $c_{1}, c_{2}>0$ e $p \in\left(1,2_{*}-1\right)$, tais que $|g(x, s)| \leq c_{1}|s|+c_{2}|s|^{p}, \forall s \in \mathbb{R}$ e $x \in \mathbb{R}^{N}$,

$\left(g_{3}\right) 2 G(x, s) \leq g(x, s) s, \forall x \in \mathbb{R}^{N}$ e $s \in \mathbb{R}$

$\left(g_{4}\right) g(x, s) s \leq \frac{1}{k} V(x) s^{2} \forall x \notin \Omega$ e $s \in \mathbb{R}$,

$\left(g_{5}\right) \frac{g(x, s)}{s}$ é não-descrescente para todo $s>0$ e não-crescente para todo $s<0$ e $x \in \mathbb{R}^{N}$.

Considere o seguinte problema

$$
\left\{\begin{array}{r}
\Delta^{2} v+V(\epsilon x) v=g(\epsilon x, v) \text { em } \mathbb{R}^{N} \\
v \in H^{2}\left(\mathbb{R}^{N}\right)
\end{array}\right.
$$

e observe que este é equivalente ao problema (2.2), onde as soluções $u_{\epsilon}$ e $v_{\epsilon}$ de (2.2) e (2.3), respectivamente, estão relacionadas por

$$
v_{\epsilon}(x)=u_{\epsilon}(\epsilon x) .
$$

Uma vez que os argumentos utilizados são variacionais, vamos introduzir o espaço adequado para tratar o problema.

Consideremos o seguinte espaço de Hilbert, $E_{\epsilon}=\left(H^{2}\left(\mathbb{R}^{N}\right),\langle\cdot, \cdot\rangle_{\epsilon}\right)$, onde

$$
\langle u, v\rangle_{\epsilon}=\int_{\mathbb{R}^{N}}(\Delta u \Delta v+V(\epsilon x) u v) d x
$$

é um produto interno que dá origem à seguinte norma

$$
\|u\|_{\epsilon}=\int_{\mathbb{R}^{N}}\left(|\Delta u|^{2}+V(\epsilon x) u^{2}\right) d x
$$

O funcional energia associado ao problema (2.3), é dado por

$$
I_{\epsilon}(u)=\frac{1}{2} \int_{\mathbb{R}^{N}}\left(|\Delta u|^{2}+V(\epsilon x) u^{2}\right) d x-\int_{\mathbb{R}^{N}} G(\epsilon x, u) d x,
$$


que está bem definido sobre $E_{\epsilon}$, devido as condições sobre $g$.

Ainda devido as condições que $g$ satisfaz, está bem definida a Variedade de Nehari associada ao problema (2.3), dada por

$$
\mathcal{N}_{\epsilon}=\left\{u \in E_{\epsilon} \backslash\{0\} ; I_{\epsilon}^{\prime}(u) u=0\right\}
$$

Lema 2.2.1. Suponha válidas as condições $\left(g_{1}\right)-\left(g_{5}\right)$ e $\left(V_{1}\right),\left(V_{3}\right)$. Então, para cada $\epsilon>0$, existem constantes positivas $\rho, r$ e uma função $v \in E_{\epsilon}$ com $\|v\|_{\epsilon}>r$, tais que:

1. $I_{\epsilon}(u) \geq \rho$ para todo $u \in E_{\epsilon}$, tal que $\|u\|_{\epsilon}=r$.

2. $I_{\epsilon}(v)<0$.

Demonstração. A demonstração desse resultado segue de maneira análoga ao Lema 1.2.3 do Capítulo 1.

Mostremos agora que $I_{\epsilon}$ satisfaz a condição de Palais-Smale.

Lema 2.2.2. Suponha válidas as condições $\left(g_{1}\right)-\left(g_{5}\right)$ e $\left(V_{1}\right)$. Então, $I_{\epsilon}$ satisfaz a condição de Palais-Smale, ou seja, se $\left(u_{n}\right)$ é uma sequência em $E_{\epsilon}$ tal que $\left(I_{\epsilon}\left(u_{n}\right)\right)$ é limitada e ainda $I_{\epsilon}^{\prime}\left(u_{n}\right) \rightarrow 0$ quando $n \rightarrow \infty$, então $\left(u_{n}\right)$ contém uma sequência fortemente convergente em $E_{\epsilon}$.

Demonstração. Mostremos primeiramente que toda sequência (PS) para $I_{\epsilon}$ é limitada em $E_{\epsilon}$.

Sem perda de generalidade, podemos supor que $\left(u_{n}\right) \subset E_{\epsilon}$ é tal que $I_{\epsilon}\left(u_{n}\right) \rightarrow d$ e $I_{\epsilon}^{\prime}\left(u_{n}\right) \rightarrow 0$. Suponhamos que exista uma subsequência de $\left(u_{n}\right)$ (denotada da mesma forma por simplicidade), tal que $\left\|u_{n}\right\|_{\epsilon} \rightarrow+\infty$. Seja $w_{n}=u_{n} /\left\|u_{n}\right\|_{\epsilon}$. Pela Observação 1.2.10, $w_{n} \rightarrow 0 \mathrm{em} E_{\epsilon}$.

Seja $t_{n} \in[0,1]$ tal que

$$
I_{\epsilon}\left(t_{n} w_{n}\right)=\max _{t \in[0,1]} I_{\epsilon}\left(t w_{n}\right) .
$$

Seja $\Omega_{\epsilon}=\frac{1}{\epsilon} \Omega$ e $\Omega_{\epsilon}^{\prime}=\frac{1}{\epsilon} \Omega^{\prime}$. Como $\left\|u_{n}\right\|_{\epsilon} \rightarrow+\infty$, para qualquer $R>0$ dado, existe um inteiro positivo $n_{0}$ tal que $R /\left\|u_{n}\right\|_{\epsilon} \leq 1$ para todo $n \geq n_{0}$. Usando a definição de $t_{n}$ e a 
condição $\left(g_{4}\right)$, segue que

$$
\begin{aligned}
I_{\epsilon}\left(t_{n} u_{n}\right) & \geq I_{\epsilon}\left(\frac{R}{\left\|u_{n}\right\|_{\epsilon}} u_{n}\right) \\
& =\frac{R^{2}}{2}\left\|w_{n}\right\|_{\epsilon}^{2}-\int_{\mathbb{R}^{N}} G\left(\epsilon x, R w_{n}\right) d x \\
& =\frac{R^{2}}{2}\left\|w_{n}\right\|_{\epsilon}^{2}-\int_{\Omega_{\epsilon}} G\left(\epsilon x, R w_{n}\right) d x-\int_{\mathbb{R}^{N} \backslash \Omega_{\epsilon}} G\left(\epsilon x, R w_{n}\right) d x \\
& \geq \frac{R^{2}}{2}\left\|w_{n}\right\|_{\epsilon}^{2}-\int_{\Omega_{\epsilon}} G\left(\epsilon x, R w_{n}\right) d x-\frac{R^{2}}{2} \int_{\mathbb{R}^{N} \backslash \Omega_{\epsilon}} \frac{V(\epsilon x)}{k} w_{n}^{2} d x \\
& \geq \frac{R^{2}}{2}\left\|w_{n}\right\|_{\epsilon}^{2}-\int_{\Omega_{\epsilon}} G\left(\epsilon x, R w_{n}\right) d x-\frac{R^{2}}{2} \int_{\mathbb{R}^{N}} \frac{V(\epsilon x)}{k} w_{n}^{2} d x \\
& =\frac{R^{2}}{2} \int_{\mathbb{R}^{N}}\left(\left|\Delta w_{n}\right|^{2}+\left(\frac{k-1}{k}\right) V(\epsilon x) w_{n}^{2}\right) d x-\int_{\Omega_{\epsilon}} G\left(\epsilon x, R w_{n}\right) d x \\
& \geq \frac{R^{2}(k-1)}{2 k}\left\|w_{n}\right\|_{\epsilon}^{2}-\int_{\Omega_{\epsilon}} G\left(\epsilon x, R w_{n}\right) d x \\
& =\frac{R^{2}(k-1)}{2 k}-\int_{\Omega_{\epsilon}} G\left(\epsilon x, R w_{n}\right) d x .
\end{aligned}
$$

Como $w_{n} \rightarrow 0$ em $E_{\epsilon}$, então $w_{n} \rightarrow 0$ em $L_{l o c}^{r}\left(\mathbb{R}^{N}\right)$, para todo $2 \leq r<2_{*}$. Então, por $\left(g_{2}\right)$, vemos que

$$
\liminf _{n \rightarrow+\infty} I\left(t_{n} u_{n}\right) \geq \frac{R^{2}(k-1)}{2 k} .
$$

Sendo $R>0$ arbitrário, resulta que

$$
\lim _{n \rightarrow+\infty} I\left(t_{n} u_{n}\right)=+\infty .
$$

Como $I_{\epsilon}(0)=0$ e $I_{\epsilon}\left(u_{n}\right) \rightarrow d$, implica $t_{n} \in(0,1)$. Portanto

$$
\left.\frac{d}{d t} I_{\epsilon}\left(t, u_{n}\right)\right|_{t=t_{n}}=0
$$

isto é,

$$
I_{\epsilon}^{\prime}\left(t_{n} u_{n}\right) u_{n}=0 .
$$

Por $\left(g_{5}\right)$, segue que $H(x, s)=g(x, s) s-2 G(x, s)$ é não-decrescente em $s>0$ e não-crescente em $s<0$. Assim,

$$
\begin{aligned}
2 I_{\epsilon}\left(t_{n} u_{n}\right) & =2 I_{\epsilon}\left(t_{n} u_{n}\right)-I_{\epsilon}^{\prime}\left(t_{n} u_{n}\right) t_{n} u_{n} \\
& =\int_{\mathbb{R}^{N}}\left(g\left(\epsilon x, t_{n} u_{n}\right) t_{n} u_{n}-2 G\left(\epsilon x, t_{n} u_{n}\right)\right) d x \\
& \leq \int_{\mathbb{R}^{N}}\left(g\left(\epsilon x, u_{n}\right) u_{n}-2 G\left(\epsilon x, u_{n}\right)\right) d x,
\end{aligned}
$$

o que implica

$$
\lim _{n \rightarrow+\infty} \int_{\mathbb{R}^{N}}\left(g\left(\epsilon x, u_{n}\right) u_{n}-2 G\left(\epsilon x, u_{n}\right)\right) d x=+\infty .
$$


Por outro lado,

$$
\int_{\mathbb{R}^{N}}\left(g\left(\epsilon x, u_{n}\right) u_{n}-2 G\left(\epsilon x, u_{n}\right)\right) d x=2 I_{\epsilon}\left(u_{n}\right)-I_{\epsilon}^{\prime}\left(u_{n}\right) u_{n}=2 d+o_{n}(1),
$$

o que contradiz (2.4). Portanto, $\left(u_{n}\right)$ é limitada em $E_{\epsilon}$.

Assim, existe $u \in H^{2}\left(\mathbb{R}^{N}\right)$ tal que $u_{n} \rightarrow u$ em $E_{\epsilon}$, a menos de subsequência. Pelas imersões de Sobolev, $u_{n} \rightarrow u$ em $L_{l o c}^{q}\left(\mathbb{R}^{N}\right), 2 \leq q<2_{*}$, e $u_{n} \rightarrow u$ em quase todo ponto de $\mathbb{R}^{N}$, quando $n \rightarrow \infty$.

Note então que $u$ é solução fraca de (2.3), pois para todo $\varphi \in C_{0}^{\infty}\left(\mathbb{R}^{N}\right)$, pela convergência fraca,

$$
\int_{\mathbb{R}^{N}}\left(\Delta u_{n} \Delta \varphi+V(\epsilon x) u_{n} \varphi\right) d x \rightarrow \int_{\mathbb{R}^{N}}(\Delta u \Delta \varphi+V(\epsilon x) u \varphi) d x
$$

e pela convergência forte em $L_{l o c}^{q}\left(\mathbb{R}^{N}\right)$ e pelo Teorema da Convergência Dominada Generalizado,

$$
\int_{\operatorname{supp}(\varphi)} g\left(\epsilon x, u_{n}\right) \varphi d x \rightarrow \int_{\operatorname{supp}(\varphi)} g(\epsilon x, u) \varphi d x
$$

Resta então apenas mostrar que

$$
\left\|u_{n}\right\|_{\epsilon}^{2}=\int_{\mathbb{R}^{N}} g\left(\epsilon x, u_{n}\right) u_{n} d x+o_{n}(1) \rightarrow \int_{\mathbb{R}^{N}} g(\epsilon x, u) u d x=\|u\|_{\epsilon}^{2},
$$

quando $n \rightarrow \infty$, pois como $E_{\epsilon}$ é um espaço de Hilbert, $u_{n} \rightarrow u$ em $E_{\epsilon}$.

Para provar isto, mostremos primeiramente que para todo $\delta>0$, existe $R=R(\delta)>0$ tal que

$$
\limsup _{n \rightarrow \infty} \int_{\mathbb{R}^{N} \backslash B_{R}}\left(\left|\Delta u_{n}\right|^{2}+V(\epsilon x) u_{n}^{2}\right) d x<\delta .
$$

Para cada $R>0$, seja $\eta_{R} \in C^{\infty}\left(\mathbb{R}^{N}\right)$ tal que $0 \leq \eta_{R} \leq 1, \eta_{R}=0$ em $B_{\frac{R}{2}}(0), \eta_{R}=1 \mathrm{em}$ $B_{R}(0)^{c},\left|\nabla \eta_{R}\right| \leq \frac{C}{R}$ e $\left|\Delta \eta_{R}\right| \leq \frac{C}{R^{2}}$. Então note que

$$
I_{\epsilon}^{\prime}\left(u_{n}\right)\left(\eta_{R} u_{n}\right)=\int_{\mathbb{R}^{N}}\left(\Delta u_{n} \Delta\left(\eta_{R} u_{n}\right)+V(\epsilon x) \eta_{R} u_{n}^{2}\right) d x-\int_{\mathbb{R}^{N}} g\left(\epsilon x, u_{n}\right) \eta_{R} u_{n} d x,
$$

o que implica

$$
\begin{gathered}
\int_{\mathbb{R}^{N}} \eta_{R}\left(\left|\Delta u_{n}\right|^{2}+V(\epsilon x) u_{n}^{2}\right) d x= \\
I_{\epsilon}^{\prime}\left(u_{n}\right)\left(\eta_{R} u_{n}\right)-\int_{\mathbb{R}^{N}}\left(u_{n} \Delta u_{n} \Delta \eta_{R}+2 \nabla u_{n} \nabla \eta_{R} \Delta u_{n}\right) d x+\int_{\mathbb{R}^{N}} g\left(\epsilon x, u_{n}\right) \eta_{R} u_{n} d x .
\end{gathered}
$$

Seja $R>0$ grande o suficiente para que $\Omega_{\epsilon} \subset B_{\frac{R}{2}}(0)$. Então, por $\left(g_{4}\right)$,

$$
\begin{gathered}
\int_{\mathbb{R}^{N}} \eta_{R}\left(\left|\Delta u_{n}\right|^{2}+V(\epsilon x) u_{n}^{2}\right) d x \leq \\
I_{\epsilon}^{\prime}\left(u_{n}\right)\left(\eta_{R} u_{n}\right)-\int_{\mathbb{R}^{N}}\left(\left|u_{n} \Delta u_{n} \Delta \eta_{R}+2 \nabla u_{n} \nabla \eta_{R} \Delta u_{n}\right|\right) d x+\frac{1}{k} \int_{\mathbb{R}^{N}} V(\epsilon x) u_{n} \eta_{R} d x,
\end{gathered}
$$

e pela Desigualdade de Hölder, temos que

$$
\begin{aligned}
\left(1-\frac{1}{k}\right) \int_{B_{R}^{c}}\left(\left|\Delta u_{n}\right|^{2}+V(\epsilon x) u_{n}^{2}\right) d x \leq & I_{\epsilon}^{\prime}\left(u_{n}\right)\left(\eta_{R} u_{n}\right)+\frac{C}{R^{2}}\left\|u_{n}\right\|_{L^{2}\left(\mathbb{R}^{N}\right)}\left\|\Delta u_{n}\right\|_{L^{2}\left(\mathbb{R}^{N}\right)} \\
& +\frac{2 C}{R}\left\|\nabla u_{n}\right\|_{L^{2}\left(\mathbb{R}^{N}\right)}\left\|\Delta u_{n}\right\|_{L^{2}\left(\mathbb{R}^{N}\right)} \\
\leq & I_{\epsilon}^{\prime}\left(u_{n}\right)\left(\eta_{R} u_{n}\right)+\frac{\tilde{C}}{R},
\end{aligned}
$$


o que implica, dado $\delta>0$, existe $R(\delta)>0$ tal que

$$
\limsup _{n \rightarrow \infty} \int_{B_{R}^{c}}\left(\left|\Delta u_{n}\right|^{2}+V(\epsilon x) u_{n}^{2}\right) d x<\frac{\delta}{2}
$$

Mostremos finalmente (2.5).

Dado $\delta>0$, seja $R(\delta)>0$ tal que

$$
\limsup _{n \rightarrow \infty} \int_{B_{R}^{c}}\left(\left|\Delta u_{n}\right|^{2}+V(\epsilon x) u_{n}^{2}\right) d x<\frac{\delta}{2} .
$$

Observe então que devido ao crescimento subcrítico de $g$ e às imersões contínuas de $H^{2}\left(B_{R}(0)^{c}\right)$ em $L^{2}\left(B_{R}(0)^{c}\right)$ e $L^{p+1}\left(B_{R}(0)^{c}\right)$, segue que

$$
\limsup _{n \rightarrow \infty} \int_{B_{R}^{c}} g\left(\epsilon x, u_{n}\right) u_{n} d x<\frac{\delta}{2}
$$

Pelas imersões compactas de Sobolev, Teorema da Convergência Dominada Generalizado e por $\left(g_{2}\right)$, segue que

$$
\int_{B_{R}} g\left(\epsilon x, u_{n}\right) u_{n} d x \rightarrow \int_{B_{R}} g(\epsilon x, u) u d x
$$

quando $n \rightarrow \infty$.

Pela integrabilidade de $x \mapsto g(\epsilon x, u(x)) u(x)$, temos que existe $R(\delta)>0$ (possivelmente maior que o anterior), tal que

$$
\int_{B_{R}^{c}} g(\epsilon x, u) u d x<\frac{\delta}{2}
$$

Logo

$$
\left|\int_{\mathbb{R}^{N}} g\left(\epsilon x, u_{n}\right) u_{n} d x-\int_{\mathbb{R}^{N}} g(\epsilon x, u) u d x\right|<\delta,
$$

se $n$ é grande o suficiente, o que encerra a demonstração.

Pelo Teorema do Passo da Montanha, para cada $\epsilon>0$, existe $v_{\epsilon}>0 \in E_{\epsilon}$ solução fraca não-trivial de (2.3), no nível minimax

$$
c_{\epsilon}=\inf _{\gamma \in \Gamma_{\epsilon}} \max _{t \in[0,1]} I_{\epsilon}(\gamma(t))
$$

onde $\Gamma_{\epsilon}=\left\{\gamma \in C^{0}\left([0,1], E_{\epsilon}\right) ; \gamma(0)=0\right.$ e $\left.I_{\epsilon}(\gamma(1))<0\right\}$.

Consideremos agora uma sequência $\epsilon_{n} \rightarrow 0$. Mostremos que a menos de subsequência, $v_{n}:=v_{\epsilon_{n}}$ é solução de

$$
\left\{\begin{aligned}
\Delta^{2} v+V\left(\epsilon_{n} x\right) v= & f(v) \text { em } \mathbb{R}^{N} \\
v & \in H^{2}\left(\mathbb{R}^{N}\right) .
\end{aligned}\right.
$$

A demonstração desse fato será dividida em uma série de lemas. O primeiro deles estabelece uma estimativa superior para $c_{\epsilon_{n}}$, a saber: 


\section{Lema 2.2.3.}

$$
\limsup _{n \rightarrow \infty} c_{\epsilon_{n}} \leq c_{0}
$$

onde $c_{\epsilon_{n}}$ e $c_{0}$ são os respectivos níveis minimax associados aos problemas (2.3) $e$

$$
\left\{\begin{array}{r}
\Delta^{2} w+V_{0} w=f(w) e m \mathbb{R}^{N} \\
w \in H^{2}\left(\mathbb{R}^{N}\right) .
\end{array}\right.
$$

Demonstração. Uma vez que o problema é invariante por translação, podemos supor sem perda de generalidade que $x_{0}=0$, onde $V\left(x_{0}\right)=\inf _{\mathbb{R}^{N}} V$. Pela Proposição 1.2.11, sabemos que existe solução não-trivial $w$ de $(2.7)$, com $I_{0}(w)=c_{0}$, onde $I_{0}$ é o funcional energia associado a (2.7). Seja $\psi \in C^{\infty}\left(\mathbb{R}^{N}\right)$ tal que $\psi \equiv 1$ em $B_{\rho}(0)$ e $\psi \equiv 0$ em $B_{2 \rho}(0)^{c}$, onde $B_{2 \rho}(0) \subset \Omega^{\prime}$.

Considere agora $w_{n}(x)=\psi\left(\epsilon_{n} x\right) w(x)$. Note que $w_{n} \rightarrow w$ em $H^{2}\left(\mathbb{R}^{N}\right)$ e em $L^{r}\left(\mathbb{R}^{N}\right)$, onde $2<r<2_{*}$ e ainda que $\operatorname{supp}\left(w_{n}\right) \subset \Omega_{\epsilon_{n}}^{\prime}$. Seja $\varphi_{\epsilon_{n}}\left(w_{n}\right)>0$ tal que $\varphi_{\epsilon_{n}}\left(w_{n}\right) w_{n} \in \mathcal{N}_{\epsilon_{n}}$. Suponhamos por um instante que $\varphi_{\epsilon_{n}}\left(w_{n}\right) \rightarrow 1$, quando $n \rightarrow \infty$. Então

$$
\begin{aligned}
c_{\epsilon_{n}} & \leq \max _{t \geq 0} I_{\epsilon_{n}}\left(t w_{n}\right) \\
& =I_{\epsilon_{n}}\left(\varphi_{\epsilon_{n}}\left(w_{n}\right) w_{n}\right) \\
& =\frac{\varphi_{\epsilon_{n}}\left(w_{n}\right)^{2}}{2} \int_{\mathbb{R}^{N}}\left(\left|\Delta w_{n}\right|^{2}+V\left(\epsilon_{n} x\right) w_{n}^{2}\right) d x-\int_{\mathbb{R}^{N}} F\left(\varphi_{\epsilon_{n}}\left(w_{n}\right) w_{n}\right) d x \\
& =I_{0}\left(\varphi_{\epsilon_{n}}\left(w_{n}\right) w_{n}\right)+\frac{1}{2} \int_{\mathbb{R}^{N}}\left(V\left(\epsilon_{n} x\right)-V(0)\right) \varphi_{\epsilon_{n}}\left(w_{n}\right)^{2} w_{n}^{2} d x .
\end{aligned}
$$

Assim, pelo Teorema da Convergência Dominada,

$$
\limsup _{n \rightarrow \infty} c_{\epsilon_{n}} \leq c_{0}
$$

Mostremos agora que $\varphi_{\epsilon_{n}}\left(w_{n}\right) \rightarrow 1$, quando $n \rightarrow \infty$.

Lembre-se que $\left.\frac{d}{d t} I_{\epsilon_{n}}\left(t w_{n}\right)\right|_{\varphi_{\epsilon_{n}}\left(w_{n}\right)}=0$, ou seja $I_{\epsilon_{n}}^{\prime}\left(\varphi_{\epsilon_{n}}\left(w_{n}\right) w_{n}\right) w_{n}=0$, o que ocorre se, e somente se,

$$
\varphi_{\epsilon_{n}}\left(w_{n}\right) \int_{\mathbb{R}^{N}}\left(\left|\Delta w_{n}\right|^{2}+V\left(\epsilon_{n} x\right) w_{n}^{2}\right) d x=\int_{\mathbb{R}^{N}} f\left(\varphi_{\epsilon_{n}}\left(w_{n}\right) w_{n}\right) w_{n} d x
$$

Usando os mesmos argumentos utilizados na demonstração do Lema 1.3.2 do Capítulo 1, provamos que $\left(\varphi_{\epsilon_{n}}\left(w_{n}\right)\right)$ é uma sequência limitada em $\mathbb{R}$.

Afirmamos agora que $\varphi_{\epsilon_{n}}\left(w_{n}\right) \nrightarrow 0$. De fato, supondo o contrário e usando a mesma argumentação empregada no Lema 1.2.5, temos

$$
\left\|w_{n}\right\|_{\epsilon_{n}}^{2}=\int_{\mathbb{R}^{N}} \frac{f\left(\varphi_{\epsilon_{n}}\left(w_{n}\right) w_{n}\right) w_{n}^{2}}{\varphi_{\epsilon_{n}}\left(w_{n}\right) w_{n}} d x \rightarrow 0, \quad \text { quando } n \rightarrow \infty,
$$

o que é impossível pois $w_{n} \rightarrow w$ e $I_{0}(w)=c_{0}>0$. Assim, existem $\alpha, \beta>0$ tais que

$$
\alpha \leq \varphi_{\epsilon_{n}}\left(w_{n}\right) \leq \beta
$$


Portanto, toda subsequência convergente de $\left(\varphi_{\epsilon_{n}}\left(w_{n}\right)\right)$ converge para 1, pois se $\bar{\varphi}$ é o limite de $\left(\varphi_{\epsilon_{k}}\left(w_{k}\right)\right)$, então

$$
\|w\|_{H^{2}\left(\mathbb{R}^{N}\right)}^{2}=\int_{\mathbb{R}^{N}} \frac{f(\bar{\varphi} w) w}{\bar{\varphi}} d x
$$

e portanto $\bar{\varphi} w \in \mathcal{N}_{0}$. Mas $w \in \mathcal{N}_{0}$ e $\log 0 \bar{\varphi}=1$.

Logo, $\varphi_{\epsilon_{n}}\left(w_{n}\right) \rightarrow 1$, pois toda subsequência possui uma subsequência convergente e toda subsequência que converge, converge para 1.

Provaremos a seguir que a sequência $\left(v_{n}\right)$ é limitada. Observamos que a demonstração desse fato não é totalmente análoga àquela do Lema 2.2.2. Isso porque, ao se tentar repetir o argumento, o limite $\int_{\Omega_{\epsilon_{n}}} G\left(\epsilon x, R w_{n}\right) d x \rightarrow 0$, quando $n \rightarrow \infty$, não decorre diretamente do fato de $w_{n} \rightarrow 0$, pois agora estamos considerando uma sequência $\epsilon_{n} \rightarrow 0$ ao invés de um $\epsilon>0$ fixo. Para contornar esta dificuldade, vamos considerar um argumento introduzido por Jeanjean e Tanaka em [22].

Lema 2.2.4. A sequência $\left(v_{n}\right)$ é limitada em $H^{2}\left(\mathbb{R}^{N}\right)$.

Demonstração. Usando o Lema 2.2.3 e o fato de $v_{n}$ ser solução de $(2.3)$, temos que $\left(I_{\epsilon_{n}}\left(v_{n}\right)\right)$ é limitada, bem como $I_{\epsilon_{n}}^{\prime}\left(v_{n}\right)=0$, para todo $n \in \mathbb{N}$. Sem perda de generalidade, podemos supor que $\left(v_{n}\right) \subset H^{2}\left(\mathbb{R}^{N}\right)$ é tal que $I_{\epsilon_{n}}\left(v_{n}\right) \rightarrow d \leq c_{0}$. Suponhamos por contradição que exista uma subsequência de $\left(v_{n}\right)$ (denotada da mesma forma por simplicidade), tal que $\left\|v_{n}\right\|_{H^{2}\left(\mathbb{R}^{N}\right)} \rightarrow+\infty$. Defina $w_{n}=v_{n} /\left\|v_{n}\right\|_{\epsilon_{n}}$. Observe primeiramente que $w_{n}$ satisfaz o seguinte problema:

$$
\Delta^{2} w_{n}+V\left(\epsilon_{n} x\right) w_{n}=\chi\left(\epsilon_{n} x\right) \frac{f\left(v_{n}\right)}{v_{n}} w_{n}+\left(1-\chi\left(\epsilon_{n} x\right)\right) \frac{\tilde{f}\left(v_{n}\right)}{v_{n}} w_{n} \quad \text { em } \mathbb{R}^{N} .
$$

Note que uma das duas situações ocorre:

$$
\begin{aligned}
& \text { i) } \limsup _{n \rightarrow \infty} \sup _{y \in \mathbb{R}^{N}} \int_{B_{1}(y)}\left|\chi\left(\epsilon_{n} x\right) w_{n}(x)\right|^{2} d x>0 ; \\
& \text { ii) } \lim _{n \rightarrow \infty} \sup _{y \in \mathbb{R}^{N}} \int_{B_{1}(y)}\left|\chi\left(\epsilon_{n} x\right) w_{n}(x)\right|^{2} d x=0 .
\end{aligned}
$$

Mostraremos porém que nenhuma destas situações de fato ocorre, o que levará a uma contradição.

Suponha que i) ocorre. Considerando uma subsequência se necessário, existe $\left(y_{n}\right) \subset \mathbb{R}^{N}$ tal que

$$
\limsup _{n \rightarrow \infty} \int_{B_{1}\left(y_{n}\right)}\left|\chi\left(\epsilon_{n} x\right) w_{n}(x)\right|^{2} d x>0 .
$$

Devido a isso, note que $B_{1}\left(y_{n}\right) \cap \Omega_{\epsilon_{n}} \neq \emptyset$, ou seja, $\operatorname{dist}\left(\epsilon_{n} y_{n}, \Omega\right)<\epsilon_{n}$. Assim, podemos supor que $\epsilon_{n} y_{n} \rightarrow x_{0} \in \bar{\Omega}$.

Seja $\bar{w}_{n}(x):=w_{n}\left(x+y_{n}\right)$ e note que $\left(\bar{w}_{n}\right)$ é limitada em $H^{2}\left(\mathbb{R}^{N}\right)$. Então existe $w_{0} \in H^{2}\left(\mathbb{R}^{N}\right)$, tal que $\bar{w}_{n} \rightarrow w_{0}$ em $H^{2}\left(\mathbb{R}^{N}\right)$, quando $n \rightarrow \infty$. Então 


$$
\chi\left(\epsilon_{n} \cdot+\epsilon_{n} y_{n}\right) \bar{w}_{n}(\cdot) \rightarrow \chi\left(x_{0}\right) w_{0}, \quad \text { em } H^{2}\left(\mathbb{R}^{N}\right),
$$

pois $\left(\chi\left(\epsilon_{n} \cdot+\epsilon_{n} y_{n}\right) \bar{w}_{n}(\cdot)\right)$ é uma sequência limitada em $H^{2}\left(\mathbb{R}^{N}\right)$ e converge pontualmente em quase todo ponto de $\mathbb{R}^{N}$, para $\chi\left(x_{0}\right) w_{0}$. Por $(2.9)$,

$$
\int_{B_{1}(0)}\left|\chi\left(x_{0}\right) w_{0}\right|^{2} d x>0
$$

o que implica $\chi\left(x_{0}\right) \neq 0$, bem como que existe um $\Gamma \subset B_{1}(0),|\Gamma|>0$, tal que

$$
w_{0}(x) \neq 0 \quad \text { para todo } x \in \Gamma \text {. }
$$

Multiplicando (2.8) por $w_{n}$ e integrando por partes, temos que

$$
1=\int_{\mathbb{R}^{N}}\left(\chi(\epsilon x) \frac{f\left(v_{n}\right)}{v_{n}} w_{n}^{2}+(1-\chi(\epsilon x)) \frac{\tilde{f}\left(v_{n}\right)}{v_{n}} w_{n}^{2}\right) d x,
$$

e assim

$$
\limsup _{n \rightarrow \infty} \int_{\mathbb{R}^{N}} \chi(\epsilon x) \frac{f\left(v_{n}\right)}{v_{n}} w_{n}^{2} d x \leq 1
$$

o que é equivalente a

$$
\limsup _{n \rightarrow \infty} \int_{\mathbb{R}^{N}} \chi\left(\epsilon x+\epsilon_{n} y_{n}\right) \frac{f\left(v_{n}\left(x+y_{n}\right)\right)}{v_{n}\left(x+y_{n}\right)} w_{n}\left(x+y_{n}\right)^{2} d x \leq 1 .
$$

Pela Observação 1.2.13, $0 \leq 2 F(s) \leq s f(s)$ para todo $s \in \mathbb{R}$. Assim,

$$
\limsup _{n \rightarrow \infty} \int_{\mathbb{R}^{N}} \chi\left(\epsilon x+\epsilon_{n} y_{n}\right) \frac{2 F\left(v_{n}\left(x+y_{n}\right)\right)}{v_{n}\left(x+y_{n}\right)^{2}} w_{n}\left(x+y_{n}\right)^{2} d x \leq 1 .
$$

Note agora que por $(2.11), v_{n}\left(x+y_{n}\right) \rightarrow+\infty$, quando $n \rightarrow \infty$, para todo $x \in \Gamma$. Pelo Lema de Fatou,

$$
\liminf _{n \rightarrow \infty} \int_{\Gamma} \chi\left(\epsilon x+\epsilon_{n} y_{n}\right) \frac{2 F\left(v_{n}\left(x+y_{n}\right)\right)}{v_{n}\left(x+y_{n}\right)^{2}} w_{n}\left(x+y_{n}\right)^{2} d x=\infty
$$

contradizendo (2.12). Logo, i) não ocorre.

Por outro lado, suponha agora que ii) ocorre. Note que $\chi\left(\epsilon_{n} \cdot\right) w_{n} \in H^{1}\left(\mathbb{R}^{N}\right)$ e $\left(\chi\left(\epsilon_{n} \cdot\right) w_{n}\right)$ é uma sequência limitada em $H^{1}\left(\mathbb{R}^{N}\right)$. Assim, $\left(\chi\left(\epsilon_{n} \cdot\right) w_{n}\right)$ é limitada em $L^{2}\left(\mathbb{R}^{N}\right)$ e $\left(\nabla\left(\chi\left(\epsilon_{n} \cdot\right) w_{n}\right)\right)$ é limitada em $L^{\frac{2 N}{N-2}}\left(\mathbb{R}^{N}\right)$. Podemos então utilizar o Lema de Lions (Lema I.1 de [23]), para concluir que

$$
\left\|\chi\left(\epsilon_{n} \cdot\right) w_{n}\right\|_{L^{r}\left(\mathbb{R}^{N}\right)} \rightarrow 0, \quad \text { quando } n \rightarrow \infty \text {, para todo } 2<r<2_{*} .
$$

Seja $t_{n} \in[0,1]$ tal que

$$
I_{\epsilon_{n}}\left(t_{n} v_{n}\right)=\max _{t \in[0,1]} I_{\epsilon_{n}}\left(t v_{n}\right)
$$


Como $\left\|v_{n}\right\|_{\epsilon_{n}} \rightarrow+\infty$, dado $R>0$, existe $n_{0} \in \mathbb{N}$ tal que $R /\left\|v_{n}\right\|_{\epsilon_{n}} \leq 1$ para todo $n \geq n_{0}$. Assim, por $\left(g_{4}\right)$, temos

$$
\begin{aligned}
I_{\epsilon_{n}}\left(t_{n} v_{n}\right) & \geq I_{\epsilon_{n}}\left(\frac{R}{\left\|v_{n}\right\|_{\epsilon_{n}}} v_{n}\right) \\
& =\frac{R^{2}}{2}\left\|w_{n}\right\|_{\epsilon_{n}}^{2}-\int_{\mathbb{R}^{N}} G\left(\epsilon_{n} x, R w_{n}\right) d x \\
& =\frac{R^{2}}{2}\left\|w_{n}\right\|_{\epsilon_{n}}^{2}-\int_{\mathbb{R}^{N}} \chi\left(\epsilon_{n} x\right) F\left(R w_{n}\right) d x-\int_{\mathbb{R}^{N}}\left(1-\chi\left(\epsilon_{n} x\right)\right) \tilde{F}\left(R w_{n}\right) d x \\
& \geq \frac{R^{2}}{2}\left\|w_{n}\right\|_{\epsilon_{n}}^{2}-\int_{\mathbb{R}^{N}} \chi\left(\epsilon_{n} x\right) F\left(R w_{n}\right) d x-\frac{R^{2}}{2} \int_{\mathbb{R}^{N}} \frac{V\left(\epsilon_{n} x\right)}{k} w_{n}^{2} d x \\
& =\frac{R^{2}}{2} \int_{\mathbb{R}^{N}}\left(\left|\Delta w_{n}\right|^{2}+\left(\frac{k-1}{k}\right) V\left(\epsilon_{n} x\right) w_{n}^{2}\right) d x-\int_{\mathbb{R}^{N}} \chi\left(\epsilon_{n} x\right) F\left(R w_{n}\right) d x \\
& \geq \frac{R^{2}(k-1)}{2 k}\left\|w_{n}\right\|_{\epsilon_{n}}^{2}-\int_{\mathbb{R}^{N}} \chi\left(\epsilon_{n} x\right) F\left(R w_{n}\right) d x \\
& =\frac{R^{2}(k-1)}{2 k}-\int_{\mathbb{R}^{N}} \chi\left(\epsilon_{n} x\right) F\left(R w_{n}\right) d x .
\end{aligned}
$$

Por $\left(f_{2}\right)-\left(f_{3}\right)$,

$$
\int_{\mathbb{R}^{N}} \chi\left(\epsilon_{n} x\right) F\left(R w_{n}\right) d x \leq \eta R^{2}\left\|w_{n}\right\|_{L^{2}\left(\mathbb{R}^{N}\right)}^{2}+C_{\eta} R^{p+1} \int_{\mathbb{R}^{N}} \chi\left(\epsilon_{n} x\right)\left|w_{n}\right|^{p+1} d x .
$$

Usando a Desigualdade de Hölder com os expoentes $p+1$ e $\frac{p+1}{p}$, e ainda (2.13), temos que

$$
\begin{aligned}
\int_{\mathbb{R}^{N}} \chi\left(\epsilon_{n} x\right) F\left(R w_{n}\right) d x & \leq \eta R^{2}\left\|w_{n}\right\|_{E_{\epsilon}}^{2}+C_{\eta} R^{p+1}\left\|\chi\left(\epsilon_{n} \cdot\right) w_{n}\right\|_{L^{p+1}\left(\mathbb{R}^{N}\right)}\left\|w_{n}\right\|_{L^{p+1}\left(\mathbb{R}^{N}\right)}^{p} \\
& \leq \eta R^{2}\left\|w_{n}\right\|_{E_{\epsilon}}^{2}+o_{n}(1) \\
& =\eta R^{2}+o_{n}(1),
\end{aligned}
$$

para todo $\eta>0$. Como $\eta>0$ é arbitrário, resulta

$$
\liminf _{n \rightarrow+\infty} I_{\epsilon_{n}}\left(t_{n} v_{n}\right) \geq \frac{R^{2}(k-1)}{2 k},
$$

para todo $R>0$. Logo

$$
\lim _{n \rightarrow+\infty} I_{\epsilon_{n}}\left(t_{n} v_{n}\right)=+\infty
$$

Sendo $I_{\epsilon_{n}}(0)=0$ e $I_{\epsilon_{n}}\left(v_{n}\right) \rightarrow d$, vemos que $t_{n} \in(0,1)$. Portanto

$$
\left.\frac{d}{d t} I_{\epsilon_{n}}\left(t, v_{n}\right)\right|_{t=t_{n}}=0
$$

isto é,

$$
I_{\epsilon_{n}}^{\prime}\left(t_{n} v_{n}\right) v_{n}=0
$$

Por $\left(g_{5}\right)$, segue que $H(x, s)=g(x, s) s-2 G(x, s)$ é não-decrescente em $s>0$ e não-crescente em $s<0$. Assim, 


$$
\begin{aligned}
2 I_{\epsilon_{n}}\left(t_{n} v_{n}\right) & =2 I_{\epsilon_{n}}\left(t_{n} v_{n}\right)-I_{\epsilon_{n}}^{\prime}\left(t_{n} v_{n}\right) t_{n} v_{n} \\
& =\int_{\mathbb{R}^{N}}\left(g\left(\epsilon x, t_{n} v_{n}\right) t_{n} u_{n}-2 G\left(\epsilon x, t_{n} v_{n}\right)\right) d x \\
& \leq \int_{\mathbb{R}^{N}}\left(g\left(\epsilon x, v_{n}\right) u_{n}-2 G\left(\epsilon x, v_{n}\right)\right) d x
\end{aligned}
$$

o que implica

$$
\lim _{n \rightarrow+\infty} \int_{\mathbb{R}^{N}}\left(g\left(\epsilon x, v_{n}\right) v_{n}-2 G\left(\epsilon x, v_{n}\right)\right) d x=+\infty
$$

Por outro lado,

$$
\int_{\mathbb{R}^{N}}\left(g\left(\epsilon_{n} x, v_{n}\right) v_{n}-2 G\left(\epsilon_{n} x, v_{n}\right)\right) d x=2 I_{\epsilon_{n}}\left(v_{n}\right)-I_{\epsilon_{n}}^{\prime}\left(v_{n}\right) v_{n}=2 d+o_{n}(1),
$$

o que contradiz (2.14).

Portanto, ii) também não ocorre e essa contradição nos leva a concluir que $\left(v_{n}\right)$ é uma sequência limitada em $H^{2}\left(\mathbb{R}^{N}\right)$.

O seguinte resultado é fundamental na argumentação que se segue.

Lema 2.2.5. Existe sequência $\left(y_{n}\right) \subset \mathbb{R}^{N}, R, \beta>0$, tais que

$$
\liminf _{n \rightarrow \infty} \int_{B_{R}\left(y_{n}\right)} v_{n}^{2} d x \geq \beta>0
$$

Demonstração. Suponha o contrário, ou seja, que

$$
\limsup _{n \rightarrow \infty} \sup _{y \in \mathbb{R}^{N}} \int_{B_{R}(y)} v_{n}^{2} d x=0 .
$$

Pelo Lemma I.1 de [23] ( $\operatorname{com} q=2$ e $\left.p=\frac{2 N}{N-2}\right), v_{n} \rightarrow 0$ em $L^{r}\left(\mathbb{R}^{N}\right)$, para $2<r<2 *$. Assim, pelo Teorema da Convergência Dominada,

$$
\int_{\mathbb{R}^{N}} g\left(\epsilon_{n} x, v_{n}\right) v_{n} d x=o_{n}(1) \quad \text { e } \int_{\mathbb{R}^{N}} G\left(\epsilon_{n} x, v_{n}\right) d x=o_{n}(1)
$$

Assim,

$$
c_{\epsilon_{n}}=I_{\epsilon_{n}}\left(v_{n}\right)=\int_{\mathbb{R}^{N}}\left(\frac{1}{2} g\left(\epsilon_{n} x, v_{n}\right) v_{n}-G\left(\epsilon_{n} x, v_{n}\right)\right) d x \rightarrow 0
$$

quando $n \rightarrow \infty$.

Por outro lado, como o valor minimax é uma função crescente com respeito ao potencial, se $d>0$ é o valor minimax associado ao problema

$$
\left\{\begin{aligned}
\Delta^{2} v+V_{0} v= & g\left(\epsilon_{n} x, v\right) \mathrm{em} \mathbb{R}^{N} \\
& v \in H^{2}\left(\mathbb{R}^{N}\right),
\end{aligned}\right.
$$

temos que $c_{\epsilon_{n}} \geq d$, para todo $n \in \mathbb{N}$. Mas isto contraria (2.15) e essa contradição prova o resultado. 
Lema 2.2.6. A sequência $\left(\epsilon_{n} y_{n}\right)$ é limitada em $\mathbb{R}^{N}$. Além disso, $\operatorname{dist}\left(\epsilon_{n} y_{n}, \Omega\right) \leq \epsilon_{n} R$, onde $R$ é como no Lema 2.2.5.

Demonstração. Para $\delta>0$ dado, seja $K_{\delta}$ uma $\delta$-vizinhança de $\Omega$. Seja $\phi \in C^{\infty}\left(\mathbb{R}^{N}\right)$ tal que, $\phi=0$ em $\Omega, \phi=1$ em $\mathbb{R}^{N} \backslash K_{\delta}, 0 \leq \phi \leq 1$ e $|\nabla \phi| \leq \frac{C}{\delta}$ e $|\Delta \phi| \leq \frac{C}{\delta^{2}}$. Seja agora $\phi_{\epsilon}(x)=\phi(\epsilon x)$ e considere $v_{n} \phi_{\epsilon_{n}}$ como função teste em (2.3). Temos então que,

$$
\int_{\mathbb{R}^{N}}\left(\Delta v_{n} \Delta\left(v_{n} \phi_{\epsilon_{n}}\right)+V\left(\epsilon_{n} x\right) v_{n}^{2} \phi_{\epsilon_{n}}\right) d x=\int_{\mathbb{R}^{N}} g\left(\epsilon_{n} x, v_{n}\right) v_{n} \phi_{\epsilon_{n}} d x
$$

o que implica

$$
\begin{aligned}
\int_{\mathbb{R}^{N}}\left(\left|\Delta v_{n}\right|^{2}+\left(V\left(\epsilon_{n} x\right)-\frac{V_{0}}{k}\right) v_{n}^{2}\right) \phi_{\epsilon_{n}} d x= & -\int_{\mathbb{R}^{N}}\left(2 \nabla v_{n} \nabla \phi_{\epsilon_{n}} \Delta v_{n}+v_{n} \Delta v_{n} \Delta \phi_{\epsilon_{n}}\right) d x \\
& +\int_{\mathbb{R}^{N}}\left(v_{n} \tilde{f}\left(v_{n}\right)-\frac{V_{0}}{k} v_{n}^{2}\right) \phi_{\epsilon_{n}} d x \\
\leq & -\int_{\mathbb{R}^{N}}\left(2 \nabla v_{n} \nabla \phi_{\epsilon_{n}} \Delta v_{n}+v_{n} \Delta v_{n} \Delta \phi_{\epsilon_{n}}\right) d x
\end{aligned}
$$

implicando que

$$
\begin{aligned}
V_{0}\left(1-\frac{1}{k}\right) \int_{\mathbb{R}^{N}} v_{n}^{2} \phi_{\epsilon_{n}} d x & \leq-\int_{\mathbb{R}^{N}}\left(2 \nabla v_{n} \nabla \phi_{\epsilon_{n}} \Delta v_{n}+v_{n} \Delta v_{n} \Delta \phi_{\epsilon_{n}}\right) d x \\
& \leq \frac{2 \epsilon_{n} C}{\delta}\left\|v_{n}\right\|_{H^{2}\left(\mathbb{R}^{N}\right)}^{2}+\frac{C \epsilon_{n}^{2}}{\delta^{2}}\left\|v_{n}\right\|_{H^{2}\left(\mathbb{R}^{N}\right)}^{2} \leq \frac{\tilde{C} \epsilon_{n}}{\delta}\left\|v_{n}\right\|_{H^{2}\left(\mathbb{R}^{N}\right)}^{2} .
\end{aligned}
$$

Se para alguma subsequência $\left(\epsilon_{k}\right)$, tivermos que $B_{R}\left(y_{\epsilon_{k}}\right) \cap \frac{K_{\delta}}{\epsilon_{k}}=\emptyset$, então

$$
V_{0}\left(1-\frac{1}{k}\right) \int_{B_{R}\left(y_{\epsilon_{k}}\right)} v_{\epsilon_{k}}^{2} d x \leq V_{0}\left(1-\frac{1}{k}\right) \int_{\mathbb{R}^{N}} v_{\epsilon_{k}}^{2} \phi_{\epsilon_{k}} d x \leq \frac{\tilde{C} \epsilon_{k}}{\delta}\left\|v_{\epsilon_{k}}\right\|_{H^{2}\left(\mathbb{R}^{N}\right)}^{2} \rightarrow 0,
$$

quando $k \rightarrow \infty$, contrariando o lema anterior.

Assim, para cada $n \in \mathbb{N}$, existe $x_{n}$ tal que $\epsilon_{n} x_{n} \in K_{\delta}$ e $\left|y_{\epsilon_{n}}-x_{n}\right|<R$. Como $\operatorname{dist}\left(\epsilon_{n} y_{\epsilon_{n}}, \Omega\right)<\epsilon_{n} R+\delta$, para todo $\delta>0$, o resultado segue.

Observação 2.2.7. Pelo lema anterior, podemos supor que $\epsilon_{n} y_{n} \in \bar{\Omega}$ para todo $n$ suficientemente grande. De fato, caso contrário, podemos considerar $\epsilon_{n}^{-1} z_{n}$ ao invés de $y_{n}$, onde $z_{n} \in \bar{\Omega}$ é tal que $\left|\epsilon_{n} y_{n}-z_{n}\right| \leq \epsilon_{n} R$. Dessa forma, a sequência $\left(z_{n} \epsilon_{n}^{-1}\right)$ terá a propriedade do Lema 2.2.5, contanto que se substitua $R$ por $2 R$.

Podemos agora demonstrar o seguinte resultado, que é essencial na demonstração do resultado principal.

Lema 2.2.8. As seguintes afirmações são verdadeiras:

i) $\lim _{n \rightarrow \infty} c_{\epsilon_{n}}=c_{0}$,

ii) $\lim _{n \rightarrow \infty} V\left(\epsilon_{n} y_{n}\right)=V_{0}$. 
Demonstração. A menos de subsequência, podemos supor que $\epsilon_{n} y_{n} \rightarrow x_{0}^{\prime} \in \bar{\Omega}$. Consideremos $w_{n}(x)=v_{n}\left(x+y_{n}\right)$, então $\liminf _{n \rightarrow \infty} \int_{B_{R}(0)} w_{n}^{2} d x \geq \beta>0$. Ainda, $\left\|w_{n}\right\|_{H^{2}\left(\mathbb{R}^{N}\right)}=\left\|v_{n}\right\|_{H^{2}\left(\mathbb{R}^{N}\right)}, \mathrm{e}$ $\operatorname{assim}\left(\left\|w_{n}\right\|_{H^{2}\left(\mathbb{R}^{N}\right)}\right)$ é limitada. Então, existe $w \in H^{2}\left(\mathbb{R}^{N}\right) \backslash\{0\}$, tal que

$$
\begin{array}{ll}
w_{n} \rightarrow w & \text { em } H^{2}\left(\mathbb{R}^{N}\right), \\
w_{n} \rightarrow w & \text { em } L_{\text {loc }}^{r}\left(\mathbb{R}^{N}\right), \text { onde } 2 \leq r<2_{*}, \\
w_{n} \rightarrow w & \text { em quase todo } x \in \mathbb{R}^{N} .
\end{array}
$$

Como $w_{n}$ satisfaz

$$
\left\{\begin{array}{l}
\Delta^{2} w_{n}+V\left(\epsilon_{n} x+\epsilon_{n} y_{n}\right) w_{n}=g\left(\epsilon_{n} x+\epsilon_{n} y_{n}, w_{n}\right) \text { em } \mathbb{R}^{N} \\
w_{n} \in H^{2}\left(\mathbb{R}^{N}\right)
\end{array}\right.
$$

então $w$ satisfaz no sentido fraco

$$
\left\{\begin{array}{l}
\Delta^{2} w+V\left(x_{0}^{\prime}\right) w=\tilde{\chi}(x) f(w)+(1-\tilde{\chi}(x)) \tilde{f}(x)=: \tilde{g}(x, w) \text { em } \mathbb{R}^{N} \\
w \in H^{2}\left(\mathbb{R}^{N}\right),
\end{array}\right.
$$

onde $\tilde{\chi}(x)=\lim _{n \rightarrow \infty} \chi\left(\epsilon_{n} x+\epsilon_{n} y_{n}\right)$, em quase todo ponto de $\mathbb{R}^{N}$.

Uma vez que o funcional associado a (2.17) possui a geometria do teorema do passo da montanha, está bem definido o nível minimax $\tilde{c}>0$ associado a (2.17). Seja ainda $\tilde{I}: H^{2}\left(\mathbb{R}^{N}\right) \rightarrow \mathbb{R}$ o funcional energia associado a $(2.17)$.

Consideremos agora o problema

$$
\left\{\begin{array}{l}
\Delta^{2} w+V\left(x_{0}^{\prime}\right) w=f(w) \text { em } \mathbb{R}^{N} \\
w \in H^{2}\left(\mathbb{R}^{N}\right)
\end{array}\right.
$$

e sejam $\bar{I}$ e $\bar{c}$ o funcional energia e o nível minimax associados a (2.18), respectivamente. Mostremos que

$$
\bar{c}=\tilde{c} .
$$

Uma vez que $\tilde{G}(x, s)=\int_{0}^{s} \tilde{g}(x, t) d t \leq F(s)$, segue que $\bar{I}(u) \leq \tilde{I}(u), \forall u \in H^{2}\left(\mathbb{R}^{N}\right)$, o que implica $\bar{c} \leq \tilde{c}$.

Para a desigualdade contrária, vamos nos utilizar do seguinte resultado, cuja prova segue as idéias do Lemma 2.2 de [18].

\section{Afirmação 2.2.9.}

$$
\tilde{I}(w) \leq \liminf _{n \rightarrow \infty} c_{\epsilon_{n}} .
$$

De fato, por estimativas elípticas, mostra-se que $w_{n}$ converge para $w$ em $C_{l o c}^{2}\left(\mathbb{R}^{N}\right)$. Assim, dado $R>0$ temos que

$$
\begin{aligned}
& \lim _{n \rightarrow \infty}\left[\frac{1}{2} \int_{B_{R}(0)}\left(\left|\Delta w_{n}\right|^{2}+V\left(\epsilon_{n} x+\epsilon_{n} y_{n}\right) w_{n}^{2}\right) d x-\int_{B_{R}(0)} G\left(\epsilon_{n} x+\epsilon_{n} y_{n}, w_{n}\right) d x\right] \\
= & \frac{1}{2} \int_{B_{R}(0)}\left(|\Delta w|^{2}+V\left(x_{0}^{\prime}\right) w^{2}\right) d x-\int_{B_{R}(0)} \tilde{G}(x, w) d x .
\end{aligned}
$$


Como a última integral converge para $\tilde{I}(w)$, quando $R \rightarrow \infty$, vemos então que dado $\delta>0$, existe $R$ grande o suficiente para que

$$
\lim _{n \rightarrow \infty}\left[\frac{1}{2} \int_{B_{R}(0)}\left(\left|\Delta w_{n}\right|^{2}+V\left(\epsilon_{n} x+\epsilon_{n} y_{n}\right) w_{n}^{2}\right) d x-\int_{B_{R}(0)} G\left(\epsilon_{n} x+\epsilon_{n} y_{n}, w_{n}\right) d x\right] \geq \tilde{I}(w)-\delta .
$$

Observe então que é suficiente mostrar que

$$
\liminf _{n \rightarrow \infty}\left[\frac{1}{2} \int_{B_{R}^{c}(0)}\left(\left|\Delta w_{n}\right|^{2}+V\left(\epsilon_{n} x+\epsilon_{n} y_{n}\right) w_{n}^{2}\right) d x-\int_{B_{R}^{c}(0)} G\left(\epsilon_{n} x+\epsilon_{n} y_{n}, w_{n}\right) d x\right] \geq-\delta,
$$

para todo $R$ suficientemente grande. Com efeito, se (2.19) valer, então

$$
\begin{aligned}
\liminf _{n \rightarrow \infty} c_{\epsilon_{n}} & =\liminf _{n \rightarrow \infty}\left[\frac{1}{2} \int_{\mathbb{R}^{N}}\left(\left|\Delta w_{n}\right|^{2}+V\left(\epsilon_{n} x+\epsilon_{n} y_{n}\right) w_{n}^{2}\right) d x-\int_{\mathbb{R}^{N}} G\left(\epsilon_{n} x+\epsilon_{n} y_{n}, w_{n}\right) d x\right] \\
& \geq \tilde{I}(w)-2 \delta
\end{aligned}
$$

para todo $\delta>0$, o que implicaria na afirmação.

Para mostrar (2.19), dado $R>0$, seja $\eta_{R} \in C^{2}\left(\mathbb{R}^{N}\right)$ tal que $\eta_{R}=1$ em $B_{R}^{c}(0), \eta_{R}=0 \mathrm{em}$ $B_{R-1}(0)$ e $\left|\nabla \eta_{R}\right|,\left|\Delta \eta_{R}\right| \leq C$, onde $C$ é uma constante que independe de $R$. Considerando então $\varphi=\eta_{R} w_{n}$ como função teste em (2.16), temos

$$
\begin{aligned}
0 & =\int_{\mathbb{R}^{N}}\left(\Delta w_{n} \Delta\left(\eta_{R} w_{n}\right)+V\left(\epsilon_{n} x+\epsilon_{n} y_{n}\right) \eta_{R} w_{n}^{2}-g\left(\epsilon_{n} x+\epsilon_{n} y_{n}, w_{n}\right) \eta_{R} w_{n}\right) d x \\
& =\int_{B_{R}^{c}}\left(\left|\Delta w_{n}\right|^{2}+V\left(\epsilon_{n} x+\epsilon_{n} y_{n}\right) w_{n}^{2}-2 G\left(\epsilon_{n} x+\epsilon_{n} y_{n}, w_{n}\right)\right) d x \\
& +\int_{B_{R}^{c}}\left(2 G\left(\epsilon_{n} x+\epsilon_{n} y_{n}, w_{n}\right)-g\left(\epsilon_{n} x+\epsilon_{n} y_{n}, w_{n}\right) w_{n}\right) d x \\
& +\int_{B_{R} \backslash B_{R-1}}\left(\Delta w_{n} \Delta\left(\eta_{R} w_{n}\right)+V\left(\epsilon_{n} x+\epsilon_{n} y_{n}\right) \eta_{R} w_{n}^{2}-g\left(\epsilon_{n} x+\epsilon_{n} y_{n}, w_{n}\right) \eta_{R} w_{n}\right) d x \\
& =A_{1, n}+A_{2, n}+A_{3, n} .
\end{aligned}
$$

Observe que o resultado estará provado se

$$
\liminf _{n \rightarrow \infty} \frac{1}{2} A_{1, n} \geq-\delta .
$$

Por $\left(g_{3}\right), A_{2, n} \leq 0$. Por outro lado, a convergência de $w_{n}$ para $w$ em $C_{l o c}^{2}\left(\mathbb{R}^{\mathbb{N}}\right)$, implica

$$
\lim _{n \rightarrow \infty} A_{3, n}=\int_{B_{R} \backslash B_{R-1}}\left(\Delta w \Delta\left(\eta_{R} w\right)+V\left(x_{0}^{\prime}\right) w^{2} \eta_{R}-\tilde{g}(x, w) \eta_{R} w\right) d x .
$$

Como a última integral, quando calculada sobre $\mathbb{R}^{N}$, converge, então para todo $R>0$ grande o suficiente, temos que

$$
\lim _{n \rightarrow \infty}\left|A_{3, n}\right| \leq \delta
$$


Portanto,

$$
\begin{aligned}
\liminf _{n \rightarrow \infty} \frac{1}{2} A_{1, n} & =\liminf _{n \rightarrow \infty}-\frac{1}{2}\left(A_{2, n}+A_{3, n}\right) \\
& \geq \liminf _{n \rightarrow \infty}-\frac{1}{2} A_{3, n} \\
& \geq-\frac{\delta}{2} .
\end{aligned}
$$

O que verifica a afirmação.

Observe então que

$$
\tilde{c} \leq \tilde{I}(w) \leq \liminf _{n \rightarrow \infty} c_{\epsilon_{n}} \leq c_{0} \leq \bar{c}
$$

Portanto

$$
\tilde{c}=\bar{c} .
$$

Assim, usando também o Lema 2.2.3,

$$
c_{0} \leq \bar{c}=\tilde{c} \leq \liminf _{n \rightarrow \infty} c_{\epsilon_{n}} \leq \limsup _{n \rightarrow \infty} c_{\epsilon_{n}} \leq c_{0},
$$

o que implica

$$
\tilde{c}=\bar{c}=c_{0}
$$

e ainda que

$$
\lim _{n \rightarrow \infty} c_{\epsilon_{n}}=c_{0},
$$

o que prova $i$ )

Agora, se ii) não valer, então $V\left(x_{0}^{\prime}\right)>V_{0}$ e assim $\tilde{c}=\bar{c}>c_{0}$, o que é uma contradição.

Pela prova do Lema 2.2.8, vemos que a menos de subsequência, $\epsilon_{n} y_{n} \rightarrow x_{0}^{\prime} \in \bar{\Omega}$. Porém, pela hipótese $\left(V_{3}\right)$ e pelas condições sobre $\Omega^{\prime}$, segue que $x_{0}^{\prime} \in \Omega^{\prime}$. De fato, supondo que $x_{0}^{\prime} \in \bar{\Omega} \backslash \Omega^{\prime}$, como $\inf _{\bar{\Omega} \backslash \Omega^{\prime}} V>\inf _{\mathbb{R}^{N}} V$, então $V\left(x_{0}^{\prime}\right)>V_{0}$, o que contradiz o item ii) do lema anterior. Com isso, vemos que a função $\tilde{\chi}(x)=1$ em todo $\mathbb{R}^{N}$, e portanto $w$ satisfaz o problema

$$
\left\{\begin{array}{r}
\Delta^{2} w+V_{0} w=f(w) \text { em } \mathbb{R}^{N} \\
w \in H^{2}\left(\mathbb{R}^{N}\right) .
\end{array}\right.
$$

\subsection{Decaimento uniforme}

Embora tenhamos obtido resultados de existência e concentração das soluções do problema modificado, até agora nada pode ser dito a respeito do problema original. Para provar que a função $v_{n}$ de fato é uma solução fraca do problema original e também que se concentra em torno de um mínimo global de $V$, precisamos estabelecer um decaimento uniforme para as translações $w_{n}$.

Lema 2.3.1. $w_{n} \rightarrow w$ em $H^{2}\left(\mathbb{R}^{N}\right)$, quando $n \rightarrow \infty$, a menos de subsequência. 
Demonstração. Pelo Lema 2.2.8, temos que $\lim _{n \rightarrow \infty} I_{\epsilon_{n}}\left(v_{n}\right)=c_{0}$. Para cada $n \in \mathbb{N}$, seja $\varphi_{0}\left(w_{n}\right)>0$ tal que $\tilde{w}_{n}=\varphi_{0}\left(w_{n}\right) w_{n} \in \mathcal{N}_{0}$, onde $\mathcal{N}_{0}$ é a variedade de Nehari associada ao problema (2.21).

Mostremos agora que $I_{0}\left(\tilde{w}_{n}\right) \rightarrow c_{0}$. De fato, pela definição de $g$, vemos que

$$
\begin{aligned}
c_{0} & \leq I_{0}\left(\tilde{w}_{n}\right) \\
& =\frac{1}{2} \int_{\mathbb{R}^{N}}\left(\left|\Delta \tilde{w}_{n}\right|^{2}+V_{0} \tilde{w}_{n}^{2}\right) d x-\int_{\mathbb{R}^{N}} F\left(\tilde{w}_{n}\right) d x \\
& \leq \frac{1}{2} \int_{\mathbb{R}^{N}}\left(\left|\Delta \tilde{w}_{n}\right|^{2}+V\left(\epsilon_{n} x+\epsilon_{n} y_{n}\right) \tilde{w}_{n}^{2}\right) d x-\int_{\mathbb{R}^{N}} G\left(\epsilon_{n} x+\epsilon_{n} y_{n}, \tilde{w}_{n}\right) d x \\
& =\frac{1}{2} \int_{\mathbb{R}^{N}}\left(\left|\Delta\left(\varphi_{0}\left(w_{n}\right) v_{n}\right)\right|^{2}+V\left(\epsilon_{n} x\right)\left(\varphi_{0}\left(w_{n}\right) v_{n}\right)^{2}\right) d x-\int_{\mathbb{R}^{N}} G\left(\epsilon_{n} x, \varphi_{0}\left(w_{n}\right) v_{n}\right) d x \\
& =I_{\epsilon_{n}}\left(\varphi_{0}\left(w_{n}\right) v_{n}\right) \\
& \leq I_{\epsilon_{n}}\left(v_{n}\right) \\
& =c_{\epsilon_{n}}=c_{0}+o_{n}(1)
\end{aligned}
$$

e isto implica $I_{0}\left(\tilde{w}_{n}\right) \rightarrow c_{0}$ quando $n \rightarrow \infty$.

Mostremos agora que $\varphi_{0}\left(w_{n}\right) \rightarrow \varphi_{0}>0$, a menos de subsequência. Primeiramente, existe $M>0$ tal que $\left|\varphi_{0}\left(w_{n}\right)\right| \leq M$, para todo $n \in \mathbb{N}$. De fato, como $w_{n} \nrightarrow 0$, segue que existe $\delta>0$ tal que $\left\|w_{n}\right\|_{H^{2}\left(\mathbb{R}^{N}\right)}>\delta$, para todo $n \in \mathbb{N}$, ao longo de uma subsequência.

Como, $I_{0}\left(\tilde{w}_{n}\right) \rightarrow c_{0}$, e $\left(\tilde{w}_{n}\right) \subset \mathcal{N}_{0}$, então repetindo os argumentos empregados na demonstração do Lema 2.2.2, é possível mostrar que $\left(\tilde{w}_{n}\right)$ é uma sequência limitada em $H^{2}\left(\mathbb{R}^{N}\right)$. Assim,

$$
\left|\varphi_{0}\left(w_{n}\right)\right| \delta<\left|\varphi_{0}\left(w_{n}\right)\right|\left\|w_{n}\right\|_{H^{2}\left(\mathbb{R}^{N}\right)}=\left\|\varphi_{0}\left(w_{n}\right) w_{n}\right\|_{H^{2}\left(\mathbb{R}^{N}\right)} \leq K,
$$

e isto implica

$$
\left|\varphi_{0}\left(w_{n}\right)\right| \leq \frac{K}{\delta}=M, \quad \forall n \in \mathbb{N} .
$$

Dessa forma, a menos de subsequência, $\varphi_{0}\left(w_{n}\right) \rightarrow \varphi_{0} \geq 0$.

Afirmamos ainda que $\varphi_{0}>0$, pois caso contrário,

$$
\left\|\tilde{w}_{n}\right\|_{H^{2}\left(\mathbb{R}^{N}\right)}=\left\|\varphi_{0}\left(w_{n}\right) w_{n}\right\|_{H^{2}\left(\mathbb{R}^{N}\right)}=\left|\varphi_{0}\left(w_{n}\right)\right|\left\|w_{n}\right\|_{H^{2}\left(\mathbb{R}^{N}\right)} \rightarrow 0,
$$

quando $n \rightarrow \infty$, o que é um absurdo. Portanto, $\tilde{w}_{n}=\varphi_{0}\left(w_{n}\right) w_{n} \rightarrow \varphi_{0} w \neq 0$ em $H^{2}\left(\mathbb{R}^{N}\right)$. O resultado segue aplicando o Lema 1.3.7 a $\left(\tilde{w}_{n}\right)$.

Assim como no Lema 1.3.8, podemos provar então que

$$
\left|w_{n}(x)\right| \rightarrow 0, \quad \text { quando }|x| \rightarrow 0 \text {, uniformemente em } n \text {. }
$$

Finalmente, vamos demonstrar que $u_{n}$ de fato é solução do problema original. Por (2.22), podemos considerar $n_{0} \in \mathbb{N}$ e $\rho>0$ tais que

$$
\left|w_{n}(x)\right|<a, \quad \forall x \in B_{\rho}(0)^{c}, \forall n \geq n_{0} .
$$


Uma vez que $x_{0}^{\prime} \in \Omega^{\prime}$ e $\epsilon_{n} y_{n} \rightarrow x_{0}^{\prime}$, é possível escolher $n_{1} \in \mathbb{N}$ tal que $B_{\rho}(0) \subset\left(\Omega_{\epsilon_{n}}^{\prime}-y_{n}\right)$, para todo $n \geq n_{1}$. Considerando então $n \geq \max \left\{n_{0}, n_{1}\right\}$, temos que

$$
g\left(\epsilon_{n} x+\epsilon_{n} y_{n}, w_{n}(x)\right)=f\left(w_{n}(x)\right), \quad \forall x \in \mathbb{R}^{N} .
$$

Portanto, para $n \geq \max \left\{n_{0}, n_{1}\right\}$ segue que $w_{n}$ satisfaz

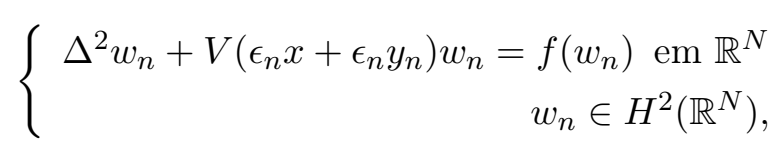

o que implica $u_{n}$ satisfaz (2.1).

Para provar que $u_{n}$ apresenta o fenômeno de concentração descrito no Teorema 2.1.1, note primeiramente que existe $\rho>0$ tal que $\left\|w_{n_{j}}\right\|_{L^{\infty}\left(\mathbb{R}^{N}\right)}>\rho$, para todo $j \in \mathbb{N}$, ao longo de uma subsequência de $\left(w_{n}\right)$. De fato, supondo que $\left\|w_{n}\right\|_{L^{\infty}\left(\mathbb{R}^{N}\right)} \rightarrow 0$, quando $n \rightarrow \infty$, então,

$$
\begin{aligned}
\left\|w_{n}\right\|_{H^{2}\left(\mathbb{R}^{N}\right)}^{2} & \leq C \int_{\mathbb{R}^{N}}\left(\left|\Delta w_{n}\right|^{2}+V\left(\epsilon_{n} x+\epsilon_{n} y_{n}\right) w_{n}\right) d x \\
& =C \int_{\mathbb{R}^{N}} f\left(w_{n}\right) w_{n} d x \\
& \leq C\left(\eta\left\|w_{n}\right\|_{L^{2}\left(\mathbb{R}^{N}\right)}^{2}+A_{\eta}\left\|w_{n}\right\|_{L^{p+1}\left(\mathbb{R}^{N}\right)}^{p+1}\right)
\end{aligned}
$$

o que implica, se $\eta<\frac{1}{2}$,

$$
\left\|w_{n}\right\|_{H^{2}\left(\mathbb{R}^{N}\right)}^{2} \leq A_{\eta}\left\|w_{n}\right\|_{L^{\infty}\left(\mathbb{R}^{N}\right)}^{p+1}\left\|w_{n}\right\|_{L^{p}\left(\mathbb{R}^{N}\right)}^{p} \rightarrow 0
$$

quando $n \rightarrow \infty$. Assim $\left\|w_{n}\right\|_{H^{2}\left(\mathbb{R}^{N}\right)}^{2} \rightarrow 0$, quando $n \rightarrow \infty$, o que contradiz o fato de $w_{n} \rightarrow w_{0}$ em $H^{2}\left(\mathbb{R}^{N}\right)$ e $w_{0} \not \equiv 0$.

Seja agora $x_{n}$ um ponto de máximo de $\left|u_{n}\right|$ em $\mathbb{R}^{N}$, então

$$
p_{n}:=\frac{x_{n}-\epsilon_{n} y_{n}}{\epsilon_{n}}
$$

é um ponto de máximo para $\left|w_{n}\right|$. Por (2.22), existe $R_{0}>0$ tal que $p_{n} \in B_{R_{0}}(0)$ para todo $n$ suficientemente grande. Então, a menos de subsequência, $p_{n} \rightarrow p_{0}$ quando $n \rightarrow \infty$. Assim

$$
x_{n}=\epsilon_{n} p_{n}+\epsilon_{n} y_{n} \rightarrow x_{0}^{\prime}, \text { quando } n \rightarrow \infty .
$$

Portanto a sequência de pontos de máximo $x_{n}$ de $\left|u_{n}\right|$ satisfaz

$$
x_{n} \rightarrow x_{0}^{\prime} \text {, quando } n \rightarrow \infty
$$

o que prova o Teorema 2.1.1. 


$\frac{10}{3}$

\section{Multiplicidade de soluções via categoria de Lusternik-Schnirelman}

\subsection{Introdução}

Nesse capítulo, vamos estudar questões sobre existência e multiplicidade de soluções nãotriviais para a seguinte família de problemas

$$
\left\{\begin{array}{c}
\epsilon^{4} \Delta^{2} u+(\lambda V(x)+1) u=f(u) \quad \mathrm{em} \mathbb{R}^{N} \\
u \in H^{2}\left(\mathbb{R}^{N}\right),
\end{array}\right.
$$

onde $\epsilon$ e $\lambda$ são parâmetros positivos. O potencial $V$ satisfaz as seguintes hipóteses:

$\left(V_{1}\right) V \in C^{1}\left(\mathbb{R}^{N}, \mathbb{R}\right), V(x) \geq 0$ para todo $x \in \mathbb{R}^{N}$, int $\left(V^{-1}(0)\right)=\Omega$ é não vazio, limitado, com fronteira suave, $0 \in \Omega$ e $V^{-1}(0)=\bar{\Omega} \cap D$, onde $D$ tem medida nula;

$\left(V_{2}\right)$ Existe $M>0$ tal que $\left|\left\{x \in \mathbb{R}^{N} ; V(x) \leq M\right\}\right|<\infty$;

e a não-linearidade $f$ satisfaz:

$\left(f_{1}\right) f \in C^{1}(\mathbb{R})$

$\left(f_{2}\right) f(0)=f^{\prime}(0)=0$ 
$\left(f_{3}\right)$ Existem constantes $c_{1}, c_{2}>0$ e $p \in\left(1,2_{*}-1\right)$, tais que $|f(s)| \leq c_{1}|s|+c_{2}|s|^{p}$, para todo $s \in \mathbb{R}$

$\left(f_{4}\right)$ Existe uma constante $\mu>2$ tal que $0<\mu F(s) \leq s f(s)$, para todo $s \in \mathbb{R} \backslash\{0\}$;

$\left(f_{5}\right) \frac{f(s)}{s}$ é crescente para todo $s>0$ e decrescente para para todo $s<0$.

O principal resultado desse capítulo é o seguinte.

Teorema 3.1.1. Suponha verdadeira as hipóteses $\left(V_{1}\right)-\left(V_{3}\right)$ e $\left(f_{1}\right)-\left(f_{5}\right)$. Existe $\epsilon^{*}>0$ tal que, para todo $\epsilon \in\left(0, \epsilon^{*}\right)$, existe $\Lambda(\epsilon)>0$ tal que (3.1) tem pelo menos cat $(\bar{\Omega})$ soluções fracas não-triviais, sempre que $\lambda \geq \Lambda(\epsilon)$.

Nesse resultado, $\operatorname{cat}(\bar{\Omega})$ denota a categoria relativa de $\bar{\Omega}$ em si próprio, como definido no Capítulo 5 de [37].

A demonstração desse resultado segue os argumentos empregados por Alves e Soares em [12]. Tratam-se de argumentos variacionais baseados em uma cuidadosa análise do comportamento dos níveis minimax do problema (3.1), quando o parâmetro $\lambda \rightarrow \infty$ e $\epsilon \rightarrow 0$.

Antes de tudo, tal como feito nos capítulos anteriores, ao invés de estudar diretamente o problema (3.1), vamos estudar o seguinte problema equivalente,

$$
\left\{\begin{array}{c}
\Delta^{2} v+(\lambda V(\epsilon x)+1) v=f(v) \quad \text { em } \mathbb{R}^{N} \\
v \in H^{2}\left(\mathbb{R}^{N}\right),
\end{array}\right.
$$

onde a equivalência entre as soluções $u_{\lambda, \epsilon}$ e $v_{\lambda, \epsilon}$ de (3.1) e (3.2), respectivamente, se dá pela relação $u_{\lambda, \epsilon}(\epsilon x)=v_{\lambda, \epsilon}(x)$.

\subsection{Condição de compacidade}

Para começar, devemos introduzir os espaços de funções adequados para posteriormente podermos definir os funcionais energia associados aos problemas com os quais queremos lidar.

Dado $\epsilon>0$, seja

$$
E=\left\{u \in H^{2}\left(\mathbb{R}^{N}\right) ; \int_{\mathbb{R}^{N}} V(\epsilon x) u^{2} d x<\infty\right\},
$$

e consideremos o seguinte produto interno em $E$,

$$
\langle u, v\rangle_{\lambda, \epsilon}=\int_{\mathbb{R}^{N}}(\Delta u \Delta v+(\lambda V(\epsilon x)+1) u v) d x
$$

que dá origem a seguinte norma

$$
\|u\|_{\lambda, \epsilon}=\left(\int_{\mathbb{R}^{N}}\left(|\Delta u|^{2}+(\lambda V(\epsilon x)+1) u^{2}\right) d x\right)^{\frac{1}{2}} .
$$

Denotemos então por $E_{\lambda, \epsilon}$ o seguinte espaço de $\operatorname{Hilbert}\left(E,\|\cdot\|_{\lambda, \epsilon}\right)$. 
Associado ao problema (3.2), temos o seguinte funcional energia $I_{\lambda, \epsilon}: E_{\lambda, \epsilon} \rightarrow \mathbb{R}$, dado por

$$
I_{\lambda, \epsilon}(v)=\frac{1}{2} \int_{\mathbb{R}^{N}}\left(|\Delta v|^{2}+(\lambda V(\epsilon x)+1) v^{2}\right) d x-\int_{\mathbb{R}^{N}} F(v) d x .
$$

Para a demonstração do Teorema 3.1.1, a seguinte proposição é de fundamental importância.

Proposição 3.2.1. Suponha $\left(V_{1}\right)-\left(V_{2}\right)$ e $\left(f_{1}\right)-\left(f_{5}\right)$ válidos, então para todo $C>0$ e $\epsilon>0$, existe $\Lambda>0$ independente de $\epsilon$, tal que $I_{\lambda, \epsilon}$ satisfaz $(P S)_{c}$ sempre que $c \leq C$ e $\lambda \geq \Lambda$.

A demonstração desse resultado seguirá de uma série de lemas que estabelecem propriedades muito importantes das sequências de Palais-Smale para $I_{\lambda, \epsilon}$.

Lema 3.2.2. Seja $\left(v_{n}\right) \subset E_{\lambda, \epsilon}$ uma sequência $(P S)_{c}$ para $I_{\lambda, \epsilon}$, então:

i) $\left(v_{n}\right)$ é limitada,

ii) $\lim \sup _{n \rightarrow \infty}\left\|v_{n}\right\|_{\lambda, \epsilon}^{2} \leq \frac{2 c \mu}{\mu-2}$,

iii) existe $c_{0}>0$ independente de $\epsilon$ tal que, se $c \neq 0$ então $c \geq c_{0}$.

Demonstração. i) Pela condição $\left(f_{4}\right)$, temos

$$
\begin{aligned}
c+o_{n}(1)+o_{n}(1)\left\|v_{n}\right\|_{\lambda, \epsilon} & \geq I_{\lambda, \epsilon}\left(v_{n}\right)-\frac{1}{\mu} I_{\lambda, \epsilon}^{\prime}\left(v_{n}\right) v_{n} \\
& \geq\left(\frac{1}{2}-\frac{1}{\mu}\right)\left\|v_{n}\right\|_{\lambda, \epsilon}^{2},
\end{aligned}
$$

o que implica $\left(\left\|v_{n}\right\|_{\lambda, \epsilon}\right)$ é limitada.

ii) Novamente por $\left(f_{4}\right)$ e por (i), temos

$$
\begin{aligned}
c & =\limsup _{n \rightarrow \infty}\left(I_{\lambda, \epsilon}\left(v_{n}\right)-\frac{1}{\mu} I_{\lambda, \epsilon}^{\prime}\left(v_{n}\right) v_{n}\right) \\
& \geq \limsup _{n \rightarrow \infty}\left(\frac{1}{2}-\frac{1}{\mu}\right)\left\|v_{n}\right\|_{\lambda, \epsilon}^{2} \\
& =\frac{\mu-2}{2 \mu} \limsup _{n \rightarrow \infty}\left\|v_{n}\right\|_{\lambda, \epsilon}^{2},
\end{aligned}
$$

o que prova ii).

iii) Lembremos primeiro que, por $\left(f_{2}\right)$ e $\left(f_{3}\right)$, temos que para todo $\eta>0$, existe $A_{\eta}>0$ tal que

$$
|f(s) s| \leq \eta|s|^{2}+A_{\eta}|s|^{p+1} .
$$

Assim, considerando $\eta<\frac{1}{2}$, existe $K>0$ tal que

$$
\begin{aligned}
I_{\lambda, \epsilon}^{\prime}(v) v & =\|v\|_{\lambda, \epsilon}^{2}-\int_{\mathbb{R}^{N}} f(v) v d x \\
& \geq\|v\|_{\lambda, \epsilon}^{2}-\eta\|v\|_{L^{2}\left(\mathbb{R}^{N}\right)}^{2}-A_{\eta}\|v\|_{L^{p}\left(\mathbb{R}^{N}\right)}^{p+1} \\
& \geq \frac{1}{2}\|v\|_{\lambda, \epsilon}^{2}-K\|v\|_{L^{p}\left(\mathbb{R}^{N}\right)}^{p+1} .
\end{aligned}
$$


Como $p>1$, existe $\delta>0$ tal que se $\|v\|_{\lambda, \epsilon}<\delta$, então $K\|v\|_{\lambda, \epsilon}^{p+1} \leq \frac{1}{4}\|v\|_{\lambda, \epsilon}^{2}$ e assim

$$
I_{\lambda, \epsilon}^{\prime}(v) v \geq \frac{1}{4}\|v\|_{\lambda, \epsilon}^{2} .
$$

Considere agora $c_{0}=\frac{\delta^{2}(\mu-2)}{2 \mu}$. Então se $c<c_{0}$ e $\left(v_{n}\right)$ é $(P S)_{c}$, então por $\left.i i\right)$

$$
\limsup _{n \rightarrow \infty}\left\|v_{n}\right\|_{\lambda, \epsilon}^{2} \leq \frac{2 c \mu}{\mu-2}<\delta^{2} .
$$

Assim para $n$ suficientemente grande, $\left\|v_{n}\right\|_{\lambda, \epsilon}<\delta$ e então

$$
\frac{1}{4}\left\|v_{n}\right\|_{\lambda, \epsilon}^{2} \leq I_{\lambda, \epsilon}^{\prime}\left(v_{n}\right) v_{n}=o_{n}(1)
$$

ou seja, $v_{n} \rightarrow 0$ em $E_{\lambda, \epsilon}$. Dessa forma, $I_{\lambda, \epsilon}\left(v_{n}\right) \rightarrow 0$, o que implica $c=0$.

Lema 3.2.3. Seja $\bar{c}>0$ fixo. Dado $\epsilon>0$ e para todo $\eta>0$, existem $\Lambda_{\eta}, R_{\eta}>0$ independentes de $\epsilon$ tais que, se $\left(v_{n}\right) e ́(P S)_{c}$ para $I_{\lambda, \epsilon} \operatorname{com} \lambda \geq \Lambda_{\eta}$ e $c \leq \bar{c}$, então

$$
\limsup _{n \rightarrow \infty} \int_{B_{R_{\eta}}^{c}}\left|v_{n}\right|^{p+1} d x \leq \eta
$$

Demonstração. Para $R>0$ e $M$ como em $\left(V_{2}\right)$, seja

$$
\begin{aligned}
& A(R)=\left\{x \in \mathbb{R}^{N} ;|x|>R \text { e } V(\epsilon x) \geq M\right\}, \\
& B(R)=\left\{x \in \mathbb{R}^{N} ;|x|>R \text { e } V(\epsilon x)<M\right\} .
\end{aligned}
$$

Temos que

$$
\begin{aligned}
\int_{A(R)} v_{n}^{2} d x & =\int_{A(R)} \frac{(\lambda V(\epsilon x)+1)}{(\lambda V(\epsilon x)+1)} v_{n}^{2} d x \\
& \leq \frac{1}{\lambda M+1} \int_{A(R)}(\lambda V(\epsilon x)+1) v_{n}^{2} d x \\
& \leq \frac{1}{\lambda M+1}\left\|v_{n}\right\|_{\lambda, \epsilon}^{2} .
\end{aligned}
$$

Pelo Lema 3.2.2 ii), temos que

$$
\limsup _{n \rightarrow \infty} \int_{A(R)} v_{n}^{2} d x \leq \frac{1}{\lambda M+1} \frac{2 c \mu}{\mu-2} .
$$

Usando a Desigualdade de Hölder e as imersões de Sobolev, se $1<r<\frac{N}{N-4}$, temos

$$
\begin{aligned}
\int_{B(R)} v_{n}^{2} d x & \leq\left(\int_{B(R)}\left|v_{n}\right|^{2 r} d x\right)^{\frac{1}{r}}\left(\int_{B(R)} d x\right)^{\frac{1}{r^{\prime}}} \\
& \leq K\left\|v_{n}\right\|_{\lambda, \epsilon}^{2}|B(R)|^{\frac{1}{r^{\prime}}}
\end{aligned}
$$


onde $r^{\prime}$ é o expoente conjugado de $r$.

Novamente devido ao Lema 3.2.2 ii), temos que

$$
\limsup _{n \rightarrow \infty} \int_{B(R)} v_{n}^{2} d x \leq K \frac{2 \bar{c} \mu}{\mu-2}|B(R)|^{\frac{1}{r^{\prime}}} .
$$
temos

Usando a desigualdade de interpolação de Gagliardo-Nirenberg com $\theta=N\left(\frac{1}{2}-\frac{1}{p+1}\right)$,

$$
\begin{aligned}
\int_{B_{R}^{c}}\left|v_{n}\right|^{p+1} d x & \leq K\left\|\Delta v_{n}\right\|_{L^{2}\left(B_{R}^{c}\right)}^{\theta(p+1)}\left\|v_{n}\right\|_{L^{2}\left(B_{R}^{c}\right)}^{(1-\theta)(p+1)} \\
& \leq K\left\|v_{n}\right\|_{\lambda, \epsilon}^{\theta(p+1)}\left(\int_{A(R)} v_{n}^{2} d x+\int_{B(R)} v_{n}^{2} d x\right)^{(1-\theta)(p+1)} .
\end{aligned}
$$

Considerando o limsup quando $n \rightarrow \infty$ em ambos os lados da desigualdade, segue que

$$
\limsup _{n \rightarrow \infty} \int_{B_{R}^{c}}\left|v_{n}\right|^{p+1} d x \leq K\left(\frac{2 \bar{c}(p+1)}{p-1}\right)^{\theta(p+1)}\left(\frac{1}{\lambda M+1} \frac{2 c \mu}{\mu-2}+K \frac{2 \bar{c} \mu}{\mu-2}|B(R)|^{\frac{1}{r^{\prime}}}\right) .
$$

Como por $\left(V_{2}\right),|B(R)| \rightarrow 0$ quando $R \rightarrow \infty$, segue que para $\eta>0$ dado, existem $\Lambda_{\eta}>0$ e $R_{\eta}>0$ independentes de $\epsilon$ tais que

$$
\int_{B_{R}^{c}}\left|v_{n}\right|^{p+1} d x<\eta
$$

contanto que $\lambda \geq \Lambda_{\eta}$ e $R>R_{\eta}$.

Lema 3.2.4. Sejam $\epsilon>0$ e $\lambda>0$ dados, existe $\delta_{0}>0$ independente de $\epsilon$ e $\lambda$, tal que se $\left(v_{n}\right)$ é uma sequência $(P S)_{c}$ para $I_{\lambda, \epsilon} \operatorname{com} c>0$, então

$$
\liminf _{n \rightarrow \infty}\left\|v_{n}\right\|_{L^{p+1}\left(\mathbb{R}^{N}\right)}^{p+1} \geq \delta_{0} c .
$$

Demonstração. Por $\left(f_{2}\right)$ e $\left(f_{3}\right)$, prova-se que para todo $\eta>0$, existe $A_{\eta}>0$ tal que

$$
\frac{1}{2} f(s) s-F(s) \leq \eta s^{2}+A_{\eta}|s|^{p+1} .
$$

Como pelo Lema 3.2.2 i), $\left(v_{n}\right)$ é uma sequência limitada, usando o Lema 3.2.2 ii), temos

$$
\begin{aligned}
c & =\lim _{n \rightarrow \infty}\left(I_{\lambda, \epsilon}\left(v_{n}\right)-\frac{1}{2} I_{\lambda, \epsilon}^{\prime}\left(v_{n}\right) v_{n}\right) \\
& =\lim _{n \rightarrow \infty} \int_{\mathbb{R}^{N}}\left(\frac{1}{2} f\left(v_{n}\right) v_{n}-F\left(v_{n}\right)\right) d x \\
& \leq \liminf _{n \rightarrow \infty} \int_{\mathbb{R}^{N}}\left(\eta\left|v_{n}\right|^{2}+A_{\eta}\left|v_{n}\right|^{p+1}\right) d x \\
& \leq \liminf _{n \rightarrow \infty}\left(\frac{2 c \mu \eta}{\mu-2}+A_{\eta}\left\|v_{n}\right\|_{L^{p+1}\left(\mathbb{R}^{N}\right)}^{p+1}\right) .
\end{aligned}
$$


Considerando agora $\eta=\frac{\mu-2}{4 \mu}$, temos que

$$
\liminf _{n \rightarrow \infty}\left\|v_{n}\right\|_{L^{p+1}\left(\mathbb{R}^{N}\right)}^{p+1} \geq \frac{1}{2 A_{\eta}} c .
$$

O resultado segue então fazendo $\delta_{0}=\frac{1}{2 A_{\eta}}$ para o $\eta$ especificado.

Lema 3.2.5. Seja $\epsilon>0, \lambda>0$ e $\left(v_{n}\right)$ uma sequência $(P S)_{c}$ para $I_{\lambda, \epsilon}$. Então a menos de subsequência, $v_{n} \rightarrow v$ em $E_{\lambda, \epsilon}$, onde $v$ é solução fraca de (3.2). Além disso, $w_{n}:=v_{n}-v$ é uma sequência $(P S)_{c^{\prime}}$ para $I_{\lambda, \epsilon}, \operatorname{com} c^{\prime}=c-I_{\lambda, \epsilon}(v)$.

Demonstração. Pelo Lema 3.2.2 i), $\left(v_{n}\right)$ é uma sequência limitada em $E_{\lambda, \epsilon}$. Logo, a menos e subsequência,

$$
\begin{aligned}
& v_{n} \rightarrow v \text { em } E_{\lambda, \epsilon} \\
& v_{n} \rightarrow v \text { em } L_{l o c}^{r}\left(\mathbb{R}^{N}\right), 2 \leq r<2_{*} \\
& v_{n} \rightarrow v \text { q.t.p. em } \mathbb{R}^{N} .
\end{aligned}
$$

Note que $v$ é ponto crítico de $I_{\lambda, \epsilon}$. De fato, se $\varphi \in C_{0}^{\infty}\left(\mathbb{R}^{N}\right)$, por (3.4) e pelo Teorema da Convergência Dominada, temos que $I_{\lambda, \epsilon}^{\prime}(v) \varphi=0$. $\operatorname{Logo} I_{\lambda, \epsilon}^{\prime}(v) \varphi=0$ para todo $\varphi \in H^{2}\left(\mathbb{R}^{N}\right)$.

Seja agora $w_{n}:=v_{n}-v$.

- $I_{\lambda, \epsilon}\left(w_{n}\right) \rightarrow c-I_{\lambda, \epsilon}(v)$, quando $n \rightarrow \infty$.

De fato, pelo Lema de Brézis-Lieb [16] e por (3.4)

$$
\begin{aligned}
I_{\lambda, \epsilon}\left(w_{n}\right)-I_{\lambda, \epsilon}\left(v_{n}\right)+I_{\lambda, \epsilon}(v)= & \frac{1}{2} \int_{\mathbb{R}^{N}}\left(\left|\Delta v_{n}-\Delta v\right|^{2}-\left|\Delta v_{n}\right|^{2}+|\Delta v|^{2}\right. \\
& \left.+(\lambda V(\epsilon x)+1)\left(\left|v_{n}-v\right|^{2}-v_{n}^{2}+v^{2}\right)\right) d x \\
& -\int_{\mathbb{R}^{N}}\left(F\left(v_{n}-v\right)-F\left(v_{n}\right)+F(v)\right) d x \\
= & \langle v, v\rangle_{\lambda, \epsilon}-\left\langle v_{n}, v\right\rangle_{\lambda, \epsilon}+o_{n}(1) \\
= & o_{n}(1) .
\end{aligned}
$$

- $\left\|I_{\lambda, \epsilon}^{\prime}\left(w_{n}\right)\right\|_{E_{\lambda, \epsilon}^{*}}=o_{n}(1)$.

Novamente pelo Lema de Brézis-Lieb e por (3.4), temos que, se $\varphi \in E_{\lambda, \epsilon}$,

$$
\begin{aligned}
I_{\lambda, \epsilon}^{\prime}\left(w_{n}\right) \varphi-I_{\lambda, \epsilon}^{\prime}\left(v_{n}\right) \varphi= & \int_{\mathbb{R}^{N}}\left(\left(\Delta v_{n}-\Delta v\right) \Delta \varphi-\Delta v_{n} \Delta \varphi\right. \\
& \left.+(\lambda V(\epsilon x)+1)\left(\left(v_{n}-v\right) \varphi-v_{n} \varphi\right)\right) d x \\
& -\int_{\mathbb{R}^{N}}\left(f\left(v_{n}-v\right) \varphi-f\left(v_{n}\right) \varphi\right) d x \\
= & -\langle v, \varphi\rangle_{\lambda, \epsilon}+\int_{\mathbb{R}^{N}} f(v) \varphi d x+o_{n}(1) \\
= & I_{\lambda, \epsilon}^{\prime}(v) \varphi+o_{n}(1)=o_{n}(1) .
\end{aligned}
$$

Finalmente podemos demonstrar a Proposição 3.2.1. 
Demonstração da Proposição 3.2.1. Seja $c_{0}$ dado pelo Lema 3.2.2 iii) e $\delta_{0}$ como no Lema 3.2.4. Considere então $\eta \in\left(0, \frac{c_{0} \delta_{0}}{2}\right)$.

Dado $C>0$, seja $\Lambda_{\eta}$ e $R_{\eta}$ dados como no Lema $3.2 .3 \operatorname{com} \bar{c}=C$.

Seja agora $\left(v_{n}\right)$ uma sequência $(P S)_{c}$ para $I_{\lambda, \epsilon} \operatorname{com} c \leq C, \lambda \geq \Lambda_{\eta}$ e $\epsilon>0$ qualquer. Pelo Lema 3.2.5, a menos de subsequência, $v_{n} \rightarrow v$ em $E_{\lambda, \epsilon}$ e $w_{n}=v_{n}-v$ é $(P S)_{c^{\prime}}$ para $I_{\lambda, \epsilon}$, onde $c^{\prime}=c-I_{\lambda, \epsilon}(v)$. Observe que, mostrando que $c^{\prime}=0$, pelo Lema 3.2.2 ii), teremos que

$$
\limsup _{n \rightarrow \infty}\left\|v_{n}-v\right\|_{\lambda, \epsilon}^{2}=\limsup _{n \rightarrow \infty}\left\|w_{n}\right\|_{\lambda, \epsilon}^{2} \leq \frac{2 c^{\prime} \mu}{\mu-2}=0
$$

e a proposição estaria demonstrada.

Suponha por contradição que $c^{\prime}>0$, então pelo Lema 3.2.2 iii), $c^{\prime} \geq c_{0}$. Pela convergência forte de $w_{n}$ para 0 em $L_{l o c}^{p+1}\left(\mathbb{R}^{N}\right)$, pelos Lemas 3.2.3 e 3.2.4, temos que

$$
\begin{aligned}
\delta_{0} c_{0} \leq \delta_{0} c^{\prime} & \leq \liminf _{n \rightarrow \infty}\left\|w_{n}\right\|_{L^{p+1}\left(\mathbb{R}^{N}\right)}^{p+1} \\
& \leq \liminf _{n \rightarrow \infty}\left\|w_{n}\right\|_{L^{p+1}\left(B_{R_{\eta}}\right)}^{p+1}+\limsup _{n \rightarrow \infty}\left\|w_{n}\right\|_{L^{p+1}\left(B_{R_{\eta}}^{c}\right)}^{p+1} \\
& \leq \eta<\frac{\delta_{0} c_{0}}{2} .
\end{aligned}
$$

Esta contradição conclui a demonstração da proposição.

Nosso próximo objetivo é estudar o comportamento de sequências de Palais-Smale, porém desta vez relacionadas a uma família de funcionais, ao invés de somente a um funcional.

Definição 3.2.6. Dado $\epsilon>0$, dizemos que uma sequência $\left(v_{n}\right) \subset H^{2}\left(\mathbb{R}^{N}\right)$ é uma sequência $(P S)_{c, \infty}$ para $\left(I_{\lambda_{n}, \epsilon}\right)$ quando:

- $\lambda_{n} \rightarrow \infty$ quando $n \rightarrow \infty$;

- $I_{\lambda_{n}, \epsilon}\left(v_{n}\right) \rightarrow c$ quando $n \rightarrow \infty$;

- $\left\|I_{\lambda_{n}, \epsilon}^{\prime}\left(v_{n}\right)\right\|_{E_{\lambda_{n}, \epsilon}^{*}} \rightarrow 0$ quando $n \rightarrow \infty$, onde $E_{\lambda_{n}, \epsilon}^{*}$ representa o espaço dual de $E_{\lambda_{n}, \epsilon}$.

Proposição 3.2.7. Seja $\epsilon>0$ fixo e suponha válidas as condições $\left(V_{1}\right),\left(V_{2}\right)$ e $\left(f_{1}\right)-\left(f_{5}\right)$. Seja $\left(v_{n}\right) \subset H^{2}\left(\mathbb{R}^{N}\right)$ uma sequência $(P S)_{c, \infty}$ para $\left(I_{\lambda_{n}, \epsilon}\right)$. Então existe uma subsequência ainda denotada por $\left(v_{n}\right)$ e uma função $v_{\epsilon} \in H^{2}\left(\mathbb{R}^{N}\right)$, tais que

i) $v_{n} \rightarrow v_{\epsilon}$ em $H^{2}\left(\mathbb{R}^{N}\right), v_{\epsilon}=0$ em $\Omega_{\epsilon}^{c}$ e $v_{\epsilon}$ é uma solução de

$$
\left\{\begin{aligned}
\Delta^{2} v+v & =f(v) \quad \text { em } \Omega_{\epsilon} \\
v & =\frac{\partial v}{\partial \nu}=0 \quad \text { em } \partial \Omega_{\epsilon}
\end{aligned}\right.
$$

onde $\Omega_{\epsilon}=\frac{1}{\epsilon} \Omega$;

ii) $\int_{\mathbb{R}^{N}} \lambda_{n} V(\epsilon x) v_{n}^{2} d x \rightarrow 0$, quando $n \rightarrow \infty$; 
iii) $\left\|v_{n}-v_{\epsilon}\right\|_{\lambda_{n}, \epsilon} \rightarrow 0$, quando $n \rightarrow \infty$.

Demonstração. Seja $\left(v_{n}\right)$ uma sequência $(P S)_{c, \infty}$ para $\left(I_{\lambda_{n}, \epsilon}\right)$. Note que $\left(\left\|v_{n}\right\|_{\lambda_{n}, \epsilon}\right)$ é limitada, pois

$$
K \geq I_{\lambda_{n}, \epsilon}\left(v_{n}\right)-\frac{1}{\mu} I_{\lambda_{n}, \epsilon}^{\prime}\left(v_{n}\right) v_{n} \geq\left(\frac{1}{2}-\frac{1}{\mu}\right)\left\|v_{n}\right\|_{\lambda_{n}, \epsilon}^{2}, \quad \forall n \in \mathbb{N} .
$$

Logo existe $v_{\epsilon} \in H^{2}\left(\mathbb{R}^{N}\right)$, tal que a menos de subsequência,

$$
\begin{aligned}
& v_{n} \rightarrow v_{\epsilon} \text { em } H^{2}\left(\mathbb{R}^{N}\right), \\
& v_{n} \rightarrow v_{\epsilon} \text { em } L_{l o c}^{r}\left(\mathbb{R}^{N}\right), 2 \leq r<2_{*}, \\
& v_{n}(x) \rightarrow v_{\epsilon}(x) \text { para quase todo } x \in \mathbb{R}^{N} .
\end{aligned}
$$

Mostremos primeiramente que $v_{\epsilon}$ é solução de (3.5).

- $v_{\epsilon} \in H_{0}^{2}\left(\Omega_{\epsilon}\right)$.

Dado $m \in \mathbb{N}$, seja $C_{m}=\left\{x \in \mathbb{R}^{N}, V(\epsilon x) \geq \frac{1}{m}\right\}$, então

$$
\int_{C_{m}} v_{n}^{2} d x \leq \int_{C_{m}} \frac{\lambda_{n} V(\epsilon x)}{\lambda_{n} V(\epsilon x)} v_{n}^{2} d x \leq \frac{m}{\lambda_{n}} \int_{C_{m}} \lambda_{n} V(\epsilon x) v_{n}^{2} d x \rightarrow 0,
$$

quando $n \rightarrow \infty$.

Pelo Lema de Fatou,

$$
\int_{C_{m}} v_{\epsilon}^{2} d x \leq \liminf _{n \rightarrow \infty} \int_{C_{m}} v_{n}^{2} d x=0, \quad \forall m \in \mathbb{N} .
$$

Como $\bigcup_{m \in \mathbb{N}} C_{m}=\mathbb{R}^{N} \backslash V_{\epsilon}^{-1}(0)$, então $v_{\epsilon}=0$ em quase todo ponto de $\mathbb{R}^{N} \backslash V_{\epsilon}^{-1}(0)$. Lembrando que $V_{\epsilon}^{-1}(0)=\Omega_{\epsilon} \cup D_{\epsilon}$ onde $D_{\epsilon}=\frac{1}{\epsilon} D$ e $\left|D_{\epsilon}\right|=|D|=0$, segue que $v_{\epsilon}(x)=0$ para quase todo $x \in \mathbb{R}^{N} \backslash \bar{\Omega}_{\epsilon}$. Também $\nabla v_{\epsilon}(x)=0$ para quase todo $x \in \mathbb{R}^{N} \backslash \bar{\Omega}_{\epsilon}$, pois para toda $\phi \in C_{0}^{\infty}\left(\mathbb{R}^{N} \backslash \bar{\Omega}_{\epsilon}\right)$, temos que

$$
\int_{\mathbb{R}^{N} \backslash \bar{\Omega}_{\epsilon}} \nabla v_{\epsilon}(x) \phi(x) d x=-\int_{\mathbb{R}^{N} \backslash \bar{\Omega}_{\epsilon}} v_{\epsilon}(x) \nabla \phi(x) d x=0 .
$$

Como $\partial \Omega_{\epsilon}$ é suave, temos que $v_{\epsilon} \in H^{2}\left(\mathbb{R}^{N} \backslash \bar{\Omega}_{\epsilon}\right)$ e $\nabla v_{\epsilon} \in H^{1}\left(\mathbb{R}^{N} \backslash \bar{\Omega}_{\epsilon}\right)$. Pelo Teorema do Traço, ver [1][Teorema 5.22], existe $K>0$ tal que

$$
\left\|v_{\epsilon}\right\|_{L^{2}\left(\partial \Omega_{\epsilon}\right)} \leq K\left\|v_{\epsilon}\right\|_{H^{2}\left(\mathbb{R}^{N} \backslash \bar{\Omega}_{\epsilon}\right)}=0
$$

e

$$
\left\|\nabla v_{\epsilon}\right\|_{L^{2}\left(\partial \Omega_{\epsilon}\right)} \leq K\left\|\nabla v_{\epsilon}\right\|_{H^{1}\left(\mathbb{R}^{N} \backslash \bar{\Omega}_{\epsilon}\right)}=0,
$$

o que mostra que $v_{\epsilon}=\nabla v_{\epsilon}=0$ em $\partial \Omega_{\epsilon}$ no sentido de traço e portanto $v_{\epsilon} \in H_{0}^{2}\left(\Omega_{\epsilon}\right)$. 
- $\int_{\Omega_{\epsilon}}\left(\Delta v_{\epsilon} \Delta \varphi+v_{\epsilon} \varphi\right) d x=\int_{\Omega_{\epsilon}} f\left(v_{\epsilon}\right) \varphi d x, \quad \forall \varphi \in H_{0}^{2}\left(\Omega_{\epsilon}\right)$.

De fato, isso segue considerando-se primeiramente $\varphi \in C_{0}^{\infty}\left(\Omega_{\epsilon}\right)$ e aplicando o Teorema da Convergência Dominada. O resultado geral segue agora pela densidade de $C_{0}^{\infty}\left(\Omega_{\epsilon}\right)$ em $H_{0}^{2}\left(\Omega_{\epsilon}\right)$.

Isso demonstra que $v_{\epsilon}$ é solução fraca de (3.5).

Para concluir o item $i$ ), resta-nos apenas mostrar que $v_{n} \rightarrow v$ em $H^{2}\left(\mathbb{R}^{N}\right)$. Isto porém segue do item iii), que será demonstrado a seguir.

Para um $R>0$ dado, consideremos os conjuntos $A(R)$ e $B(R)$ como dados na demonstração do Lema 3.2.3. Pela limitação da sequência $\left(v_{n}\right)$ em $H^{2}\left(\mathbb{R}^{N}\right)$, temos as seguintes estimativas

$$
\begin{gathered}
\int_{A(R)} v_{n}^{2} d x \leq \frac{K}{\lambda_{n} M+1} \\
\int_{B(R)} v_{n}^{2} d x \leq K|B(R)|^{\frac{1}{r^{\prime}}},
\end{gathered}
$$

onde $r^{\prime}$ é o expoente conjugado de $r \in\left(1, \frac{N}{N-4}\right)$ e $M$ é como na condição $\left(V_{2}\right)$.

Isso mostra que se $\eta>0$ é dado, existem $\Lambda_{\eta}, R_{\eta}$ tais que, se $R>R_{\eta}$ e $\lambda_{n} \geq \Lambda_{\eta}$, temos

$$
\int_{B_{R}^{c}} v_{n}^{2} d x<\eta
$$

Da mesma forma que no Lema 3.2.3, mostra-se que existem $R_{\eta}, \Lambda_{\eta}>0$, maiores que os anteriores, tais que

$$
\int_{B_{R}^{c}}\left|v_{n}\right|^{p} d x<\eta
$$

contanto que $\lambda_{n} \geq \Lambda_{\eta}$ e $R>R_{\eta}$. Agora, pela imersão contínua de $H^{2}\left(B_{R}(0)^{c}\right)$ tanto em $L^{2}\left(B_{R}(0)^{c}\right)$, quanto em $L^{p+1}\left(B_{R}(0)^{c}\right)$, usando a hipótese $\left(f_{3}\right)$, segue que para $R>R_{\eta}$ e $\lambda_{n}>\Lambda_{\eta}$

$$
\int_{B_{R}^{c}} f\left(v_{n}\right) v_{n} d x<K \eta
$$

com $K$ independente de $\eta$.

Pela integrabilidade de $f(v(\cdot))$ em $\mathbb{R}^{N}$, para o $\eta$ dado, existe $R_{\eta}>0$ maior que o anterior, tal que

$$
\int_{B_{R}^{c}} f(v) v d x<K \eta
$$

se $R>R_{\eta}$.

Com isso, para todo $n$ suficientemente grande e $R \geq R_{\eta}$, usando a convergência forte em $L_{l o c}^{q}\left(\mathbb{R}^{N}\right)$ de $v_{n}$ para $v$, com $2 \leq q<2_{*}$, temos 


$$
\begin{aligned}
\left\|v_{n}-v\right\|_{H^{2}\left(\mathbb{R}^{N}\right)}^{2} & \leq\left\|v_{n}-v\right\|_{\lambda_{n}, \epsilon}^{2} \\
& =\left\|v_{n}\right\|_{\lambda_{n}, \epsilon}^{2}+\|v\|_{\lambda_{n}, \epsilon}^{2}-2 \int_{\mathbb{R}^{N}}\left(\Delta v_{n} \Delta v+v_{n} v\right) d x \\
& =\left\|v_{n}\right\|_{\lambda_{n}, \epsilon}^{2}+\|v\|_{H^{2}\left(\mathbb{R}^{N}\right)}^{2}-2\|v\|_{H^{2}\left(\mathbb{R}^{N}\right)}^{2}+o_{n}(1) \\
& =\int_{\mathbb{R}^{N}} f\left(v_{n}\right) v_{n} d x-\int_{\mathbb{R}^{N}} f(v) v d x+o_{n}(1) \\
& =\left(\int_{B_{R}} f\left(v_{n}\right) v_{n} d x-\int_{B_{R}} f(v) v d x\right)+\left(\int_{B_{R}^{c}} f\left(v_{n}\right) v_{n} d x-\int_{B_{R}^{c}} f(v) v d x\right) \\
& <2 K \eta+o_{n}(1) .
\end{aligned}
$$

Como $\eta>0$ é arbitrário, resulta que $v_{n} \rightarrow v$ em $H^{2}\left(\mathbb{R}^{N}\right)$ quando $n \rightarrow \infty$ e ainda que

$$
\left\|v_{n}-v\right\|_{\lambda_{n}, \epsilon} \rightarrow 0, \quad \text { quando } n \rightarrow \infty .
$$

Para verificar o item ii), basta observar que

$$
\int_{\mathbb{R}^{N}} \lambda_{n} V(\epsilon x) v_{n}^{2} d x=\int_{\mathbb{R}^{N}} \lambda_{n} V(\epsilon x)\left|v_{n}-v\right|^{2} d x \leq\left\|v_{n}-v\right\|_{\lambda_{n}, \epsilon}^{2} \rightarrow 0
$$

quando $n \rightarrow \infty$.

\subsection{Comportamento dos níveis minimax}

Nessa seção vamos estudar o comportamento dos níveis minimax do funcional $I_{\lambda, \epsilon}$ com respeito a $\lambda$ e $\epsilon$. Vamos começar por algumas definições e notações.

Seja

$$
\mathcal{N}_{\lambda, \epsilon}=\left\{v \in E_{\lambda, \epsilon} \backslash\{0\} ; \quad I_{\lambda, \epsilon}^{\prime}(v) v=0\right\},
$$

a variedade de Nehari e $c_{\lambda, \epsilon}$ o nível minimax associados ao funcional $I_{\lambda, \epsilon}$. Pela hipótese $\left(f_{5}\right)$, pode-se mostrar tal como nos capítulos 1 e 2 , que $c_{\lambda, \epsilon}=\inf _{\mathcal{N}_{\lambda, \epsilon}} I_{\lambda, \epsilon}$.

De acordo com a Proposição 3.2.7, vemos que o problema (3.2) tem como problema limite, quando $\lambda \rightarrow \infty$, a equação (3.5). Assoaciados a ele, para cada $\epsilon>0$, temos o funcional energia

$$
I_{\epsilon}(v)=\frac{1}{2} \int_{\Omega_{\epsilon}}\left(|\Delta v|^{2}+v^{2}\right) d x-\int_{\Omega_{\epsilon}} F(v) d x
$$

que está bem definido sobre o espaço $H_{0}^{2}\left(\Omega_{\epsilon}\right)$. Analogamente, denotamos por $\mathcal{N}_{\epsilon}$ e $c(\epsilon, \Omega)$, a Variedade de Nehari e o nível minimax assoaciados a $I_{\epsilon}$, respectivamente. Ainda por $\left(f_{5}\right)$, temos que $c(\epsilon, \Omega)=\inf _{\mathcal{N}_{\epsilon}} I_{\epsilon}$. Seja ainda $r>0$ tal que $B_{r}(0) \subset \Omega$, que existe graças a $\left(V_{1}\right)$. Consideremos o seguinte funcional,

$$
I_{\epsilon, B_{r}}(v)=\frac{1}{2} \int_{B_{\frac{r}{\epsilon}}}\left(|\Delta v|^{2}+v^{2}\right) d x-\int_{B_{\frac{r}{\epsilon}}} F(v) d x,
$$


que está bem definido sobre $H_{0}^{2}\left(B_{\frac{r}{\epsilon}}(0)\right)$. Denotemos ainda por $\mathcal{N}_{\epsilon, B_{r}}$ e $c\left(\epsilon, B_{r}\right)$ a Variedade de Nehari e o nível minimax associados ao funcional $I_{\epsilon, B_{r}}$. Novamente por $\left(f_{5}\right)$, temos que

$$
c\left(\epsilon, B_{r}\right)=\inf _{\mathcal{N}_{\epsilon, B_{r}}} I_{\epsilon, B_{r}} .
$$

Observe que, como $B_{\frac{r}{\epsilon}}(0) \subset \Omega_{\epsilon}$, então toda função de $H_{0}^{2}\left(B_{\frac{r}{\epsilon}}(0)\right)$ pode ser estendida a uma de $H_{0}^{2}\left(\Omega_{\epsilon}\right)$. Logo vale a relação

$$
c(\epsilon, \Omega) \leq c\left(\epsilon, B_{r}\right), \quad \text { para todo } \epsilon>0 .
$$

Lema 3.3.1. Seja $v \in \mathcal{N}_{\lambda, \epsilon}$, então existe $\delta>0$ independente de $\lambda$ e $\epsilon$, tal que

$$
\|v\|_{H^{2}\left(\mathbb{R}^{N}\right)}^{2} \geq \delta
$$

Demonstração. Por $\left(f_{2}\right)$ e $\left(f_{3}\right)$, existe $C>0$ tal que

$$
|f(s)| \leq \frac{1}{2}|s|+C|s|^{p} \quad \text { para todo } s \in \mathbb{R} .
$$

Então se $v \in \mathcal{N}_{\lambda, \epsilon}$, temos que

$$
\|v\|_{\lambda, \epsilon}^{2}=\int_{\mathbb{R}^{N}} f(u) u d x \leq \frac{1}{2}\|v\|_{L^{2}\left(\mathbb{R}^{N}\right)}^{2}+C\|v\|_{L^{p+1}\left(\mathbb{R}^{N}\right)}^{p+1} .
$$

Usando a última desigualdade, as imersões de Sobolev e o fato de $\|v\|_{H^{2}\left(\mathbb{R}^{N}\right)} \leq\|v\|_{\lambda, \epsilon}$ $\forall v \in E_{\lambda, \epsilon}$, segue que

$$
\frac{1}{2}\|v\|_{H^{2}\left(\mathbb{R}^{N}\right)}^{2} \leq C\|v\|_{H^{2}\left(\mathbb{R}^{N}\right)}^{p+1} .
$$

Como $2<p+1$, por (3.6), segue a existência de tal $\delta$.

O próximo resultado descreve o comportamento do nível minimax $c_{\lambda, \epsilon}$, quando $\lambda \rightarrow \infty$.

Lema 3.3.2. Dado $\epsilon>0$, vale o seguinte limite

$$
\lim _{\lambda \rightarrow \infty} c_{\lambda, \epsilon}=c(\epsilon, \Omega)
$$

Demonstração. Seja $\lambda_{n} \rightarrow \infty$ uma sequência qualquer. Pela geometria do funcional $I_{\lambda_{n}, \epsilon} \mathrm{e}$ também pela Proposição 3.2.1, podemos usar o Teorema do Passo da Montanha para obter uma sequência $\left(v_{n}\right) \subset H^{2}\left(\mathbb{R}^{N}\right)$, tal que

$$
I_{\lambda_{n}, \epsilon}\left(v_{n}\right)=c_{\lambda_{n}, \epsilon} \quad \text { e } \quad I_{\lambda_{n}, \epsilon}^{\prime}\left(v_{n}\right)=0 .
$$

Como toda função de $H_{0}^{2}\left(\Omega_{\epsilon}\right)$ pode ser estendida a uma de $H^{2}\left(\mathbb{R}^{N}\right)$ e também pelas definições de $c_{\lambda_{n}, \epsilon}$ e $c(\epsilon, \Omega)$, temos que

$$
c_{\lambda_{n}, \epsilon} \leq c(\epsilon, \Omega), \quad \text { para todo } n \in \mathbb{N} .
$$


Isso implica $\left(I_{\lambda_{n}, \epsilon}\left(v_{n}\right)\right)$ é uma sequência limitada em $\mathbb{R}$. Portanto, existe uma subsequência, que continuaremos a denotar por $\left(v_{n}\right)$, tal que

$$
I_{\lambda_{n}, \epsilon}\left(v_{n}\right) \rightarrow c \in[0, c(\epsilon, \Omega)] \quad \text { e } \quad I_{\lambda_{n}, \epsilon}^{\prime}\left(v_{n}\right)=0 .
$$

Então, $\left(v_{n}\right)$ é uma sequência $(P S)_{c, \infty}$ para $\left(I_{\lambda_{n}, \epsilon}\right)$. Assim, pela Proposição 3.2.7, existe uma subsequência, que continuaremos a denotar por $\left(v_{n}\right)$, e $v \in H^{2}\left(\mathbb{R}^{N}\right)$, tais que

$$
v_{n} \rightarrow v \quad \text { em } H^{2}\left(\mathbb{R}^{N}\right) \quad \text { e } \quad v=0 \quad \text { em } \mathbb{R}^{N} \backslash \Omega_{\epsilon} .
$$

Ainda pela Proposição 3.2.7, $v$ satisfaz o problema (3.5).

Pelo Lema 3.3.1, temos ainda que $v \neq 0$ em $H_{0}^{2}\left(\Omega_{\epsilon}\right)$. Assim,

$$
I_{\epsilon}(v) \geq c(\epsilon, \Omega)
$$

Por outro lado,

$$
I_{\epsilon}(v)=\lim _{n \rightarrow \infty} I_{\lambda_{n}, \epsilon}\left(v_{n}\right)=\lim _{n \rightarrow \infty} c_{\lambda_{n}, \epsilon} \leq c(\epsilon, \Omega) .
$$

De (3.8) e (3.10), segue que

$$
\lim _{n \rightarrow \infty} c_{\lambda_{n}, \epsilon}=c(\epsilon, \Omega),
$$

e assim o resultado segue.

Como enunciado no Teorema 3.1.1, queremos obter ao menos cat $(\bar{\Omega})$ soluções fracas nãotriviais para o problema (3.2), contanto que $\epsilon$ seja suficientemente pequeno e $\lambda$ suficientemente grande. Isso será feito utilizando-se o seguinte resultado abstrato enunciado e provado por Willem em [37].

Teorema 3.3.3. Seja $X$ um espaço de Banach, $\psi \in C^{2}(X, \mathbb{R}), V:=\{v \in X ; \quad \psi(v)=1\}$ e ainda $\psi^{\prime}(v) \neq 0$, para todo $v \in V$. Se $\left.\varphi\right|_{V}$ é limitado inferiormente e satisfaz $(P S)_{c}$ para todo $c \in\left[\inf _{V} \varphi, d\right]$, então $\varphi^{d}$ tem pelo menos cat $\left(\varphi^{d}\right)$ pontos críticos de $\left.\varphi\right|_{V}$, onde $\varphi^{d}=\{v \in V ; \varphi(v) \leq d\}$.

Iremos aplicar esse resultado para o contexto em que $X=E_{\lambda, \epsilon}, V=\mathcal{N}_{\lambda, \epsilon}$ e $\varphi=I_{\lambda, \epsilon}$. Note que por $\left(f_{1}\right), I_{\lambda, \epsilon}$ é $C^{2}\left(E_{\lambda, \epsilon}, \mathbb{R}\right)$. Observe então que por um lado o resultado abstrato do qual dispomos trabalha com a categoria do conjunto $I_{\lambda, \epsilon}^{d} \subset H^{2}\left(\mathbb{R}^{N}\right)$, enquanto que o que desejamos é estimar o número de soluções de (3.2) pela categoria de $\bar{\Omega} \subset \mathbb{R}^{N}$. Isso nos motiva a procurar uma correlação entre $\operatorname{cat}\left(I_{\lambda, \epsilon}^{d}\right)$ e $\operatorname{cat}(\bar{\Omega})$, o que será feito através de aplicações entre subconjuntos de $H^{2}\left(\mathbb{R}^{N}\right)$ e de $\mathbb{R}^{N}$. Para isso será necessário "representar" uma função de $H^{2}\left(\mathbb{R}^{N}\right)$ por um ponto de $\mathbb{R}^{N}$, o que será feito pela função "baricentro" que será definida a seguir.

Seja $u \in H^{2}\left(\mathbb{R}^{N}\right) \backslash\{0\}$ uma função com suporte compacto, definimos então o seu baricentro $\beta(u) \in \mathbb{R}^{N}$ por

$$
\beta(u)=\frac{\int_{\mathbb{R}^{N}} x|u(x)|^{2} d x}{\int_{\mathbb{R}^{N}}|u(x)|^{2} d x} .
$$


Para podermos definir o baricentro de qualquer função em $H^{2}\left(\mathbb{R}^{N}\right)$, consideremos $R>0$ tal que $\Omega \subset B_{R}(0)$, de maneira que $\Omega_{\epsilon} \subset B_{\frac{R}{\epsilon}}(0)$. Consideremos a função

$$
\xi_{\epsilon}(t)= \begin{cases}1, & \text { se } 0 \leq t \leq \frac{R}{\epsilon} \\ 0, & \text { se } t \geq \frac{R}{\epsilon}\end{cases}
$$

Para $u \in H^{2}\left(\mathbb{R}^{N}\right) \backslash\{0\}$, seja

$$
\beta_{\epsilon}(u)=\frac{\int_{\mathbb{R}^{N}} x \xi_{\epsilon}(|x|)|u(x)|^{2} d x}{\int_{\mathbb{R}^{N}}|u(x)|^{2} d x} .
$$

Note que $\beta_{\epsilon}$ está bem definida, uma vez que para toda $u \in H^{2}\left(\mathbb{R}^{N}\right) \backslash\{0\}$,

$$
\left|\beta_{\epsilon}(u)\right|=\frac{\left.\left|\int_{\mathbb{R}^{N}} x \xi_{\epsilon}(|x|)\right| u(x)\right|^{2} d x \mid}{\|u\|_{L^{2}\left(\mathbb{R}^{N}\right)}^{2}} \leq \frac{N \int_{\mathbb{R}^{N}}|x| \xi_{\epsilon}(|x|)|u(x)|^{2} d x}{\|u\|_{L^{2}\left(\mathbb{R}^{N}\right)}^{2}} \leq N \frac{R}{\epsilon} .
$$

Para cada $y \in \mathbb{R}^{N}$ e $R>r$, definamos

$$
A_{R, r, y}=B_{R}(y) \backslash \bar{B}_{r}(y)
$$

Seja ainda $\alpha(R, r, \epsilon, y)$ o seguinte número

$$
\alpha(R, r, \epsilon, y)=\inf \left\{\hat{I}_{\epsilon, y}(v) ; \quad \beta(v)=y \text { e } v \in \hat{\mathcal{N}}_{\epsilon, y}\right\}
$$

onde

$$
\hat{I}_{\epsilon, y}(v)=\frac{1}{2} \int_{A_{\frac{R}{\epsilon}, \frac{r}{\epsilon}, y}}\left(|\Delta v|^{2}+v^{2}\right) d x-\int_{A_{\frac{R}{\epsilon}, \frac{r}{\epsilon}, y}} F(v) d x,
$$

está bem definido sobre $H_{0}^{2}\left(A_{\frac{R}{\epsilon}, \frac{r}{\epsilon}, y}\right)$ e

$$
\hat{\mathcal{N}}_{\epsilon, y}=\left\{v \in H_{0}^{2}\left(A_{\frac{R}{\epsilon}, \frac{r}{\epsilon}, y}\right) ; \quad v \neq 0 \text { e } \hat{I}_{\epsilon, y}^{\prime}(v) v=0\right\} .
$$

Denotaremos ainda $\alpha(R, r, \epsilon, 0)=\alpha(R, r, \epsilon), \hat{I}_{\epsilon, 0}=\hat{I}_{\epsilon}$ e $\hat{\mathcal{N}}_{\epsilon, 0}=\hat{\mathcal{N}}_{\epsilon}$.

\section{Lema 3.3.4.}

$$
\lim _{\epsilon \rightarrow 0} \alpha(R, r, \epsilon)>c_{\infty},
$$

onde $c_{\infty}$ é o valor minimax associado ao funcional

$$
I_{\infty}(v)=\frac{1}{2} \int_{\mathbb{R}^{N}}\left(|\Delta v|^{2}+v^{2}\right) d x-\int_{\mathbb{R}^{N}} F(v) d x
$$

definido em $H^{2}\left(\mathbb{R}^{N}\right)$.

Demonstração. Uma vez que toda função de $H_{0}^{2}\left(A_{\frac{R}{\epsilon}, \frac{r}{\epsilon}, 0}\right)$ pode ser estendida a uma de $H^{2}\left(\mathbb{R}^{N}\right)$ por 0 em $\mathbb{R}^{N} \backslash A_{\frac{R}{\epsilon}, \frac{r}{\epsilon}, 0}$, então vale o seguinte

$$
\alpha(R, r, \epsilon) \geq c_{\infty}
$$


Suponha por contradição que exista $\epsilon_{n} \rightarrow 0$ tal que $\alpha\left(R, r, \epsilon_{n}\right) \rightarrow c_{\infty}$. Então existe $\left(u_{n}\right) \subset H^{2}\left(\mathbb{R}^{N}\right)$ tal que, $\beta\left(u_{n}\right)=0, u_{n} \in \hat{\mathcal{N}}_{\epsilon_{n}}$ e $\hat{I}_{\epsilon_{n}}\left(u_{n}\right) \rightarrow c_{\infty}$. Como $I_{\infty}$ e $\hat{I}_{\epsilon_{n}}$ coincidem em $H_{0}^{2}\left(A_{\frac{R}{\epsilon_{n}}, \frac{r}{\epsilon_{n}}, 0}\right)$, segue que $I_{\infty}\left(u_{n}\right) \rightarrow c_{\infty}$ e $\left(u_{n}\right) \subset \mathcal{N}_{\infty}$, onde $\mathcal{N}_{\infty}$ é a variedade de Nehari associada a $I_{\infty}$.

Pelo Princípio Variacional de Ekeland, existe $\left(\bar{u}_{n}\right) \subset \mathcal{N}_{\infty}$ com $\left\|u_{n}-\bar{u}_{n}\right\|_{H^{2}\left(\mathbb{R}^{N}\right)}<\frac{1}{n}$ e ainda $I_{\infty}\left(\bar{u}_{n}\right) \rightarrow c_{\infty}$ e $I_{\infty}^{\prime}\left(\bar{u}_{n}\right) \rightarrow 0$, quando $n \rightarrow \infty$. Por argumentos conhecidos, prova-se que $\left(\bar{u}_{n}\right)$ é limitada em $H^{2}\left(\mathbb{R}^{N}\right)$ e então existe $u$ tal que $\bar{u}_{n} \rightarrow u$ em $H^{2}\left(\mathbb{R}^{N}\right)$. Assim, também $u_{n} \rightarrow u \mathrm{em} H^{2}\left(\mathbb{R}^{N}\right)$.

Afirmamos que $u=0$ em $H^{2}\left(\mathbb{R}^{N}\right)$. De fato, pela convergência fraca, temos que $u_{n}(x) \rightarrow u(x)$ para quase todo $x \in \mathbb{R}^{N}$. Como $\operatorname{supp}\left(u_{n}\right) \subset A_{\frac{R}{\epsilon_{n}}, \frac{r}{\epsilon_{n}}, 0}$, então para quase todo $x \in \mathbb{R}^{N}$, se $n$ é suficientemente grande, $u_{n}(x)=0$, de modo que $u(x)=0$.

Usando o Lema de Lions (Lemma I.1 de [23]) e mais o fato de $I_{\infty}\left(\bar{u}_{n}\right) \rightarrow c_{\infty}>0$, temos a existência de $R_{1}, \eta>0$ e $\left(y_{n}\right) \subset \mathbb{R}^{N}$, tais que

$$
\liminf _{n \rightarrow \infty} \int_{B_{R_{1}}\left(y_{n}\right)}\left|\bar{u}_{n}\right|^{2} d x \geq \eta
$$

Observe que $\left|y_{n}\right| \rightarrow \infty$, pois do contrário, usando as imersões de Sobolev, concluiríamos que $u \neq 0$ em $H^{2}\left(\mathbb{R}^{N}\right)$. Defina agora $v_{n}(x)=\bar{u}_{n}\left(x+y_{n}\right)$. Como o funcional $I_{\infty}$ é invariante por translações, temos que

$$
I_{\infty}\left(v_{n}\right) \rightarrow c_{\infty} \quad \text { e } \quad I_{\infty}^{\prime}\left(v_{n}\right) \rightarrow 0
$$

quando $n \rightarrow \infty$. Assim como $\left(\bar{u}_{n}\right),\left(v_{n}\right)$ é limitada em $H^{2}\left(\mathbb{R}^{N}\right)$, de modo que existe $\Psi$ tal que $v_{n} \rightarrow \Psi$ em $H^{2}\left(\mathbb{R}^{N}\right)$. Pelas imersões de Sobolev e por (3.10), temos que $\Psi \neq 0$ em $H^{2}\left(\mathbb{R}^{N}\right)$. Agora, analogamente à Proposição 1.2.11 do Capítulo 1, prova-se que $v_{n} \rightarrow \Psi$ em $H^{2}\left(\mathbb{R}^{N}\right)$ e ainda que $I_{\infty}(\Psi)=c_{\infty}$ e $I_{\infty}^{\prime}(\Psi)=0$.

Com isso, podemos afirmar então que

$$
u_{n}(x)=w_{n}(x)+\Psi\left(x-y_{n}\right),
$$

onde $w_{n} \rightarrow 0$ em $H^{2}\left(\mathbb{R}^{N}\right)$.

Como $I_{\infty}$ é rotacionalmente invariante, podemos supor sem perda de generalidade que

$$
y_{n}=\left(y_{n}^{1}, 0, \ldots, 0\right)
$$

com $y_{n}^{1}<0$, para todo $n \in \mathbb{N}$.

Seja $M=\int_{\mathbb{R}^{N}}|\Delta \Psi|^{2} d x$. Obviamente $M>0$. Uma vez que $\left\|w_{n}\right\|_{H^{2}\left(\mathbb{R}^{N}\right)} \rightarrow 0$, temos então que

$$
\int_{B_{\frac{r}{\epsilon_{n}}}\left(y_{n}\right)}\left|\Delta\left(w_{n}(x)+\Psi\left(x-y_{n}\right)\right)\right|^{2} d x \rightarrow M, \quad \text { quando } n \rightarrow \infty .
$$

Disto segue que

$$
\int_{\Theta_{n}}\left|\Delta u_{n}\right|^{2} d x \rightarrow M, \quad \text { quando } n \rightarrow \infty
$$


onde $\Theta_{n}=B_{\frac{r}{2 \epsilon_{n}}}\left(y_{n}\right) \cap\left(B_{\frac{R}{\epsilon_{n}}}(0) \backslash B_{\frac{r}{\epsilon_{n}}}(0)\right)$. Assim

$$
\int_{\Sigma_{n}}\left|\Delta u_{n}\right|^{2} d x \rightarrow 0, \quad \text { quando } n \rightarrow \infty
$$

onde $\Sigma_{n}=\left(B_{\frac{R}{\epsilon_{n}}}(0) \backslash B_{\frac{r}{\epsilon_{n}}}(0)\right) \backslash B_{\frac{r}{2 \epsilon_{n}}}\left(y_{n}\right)$.

Como $\beta\left(u_{n}\right)=0$, temos que

$$
0=\int_{A_{\frac{R}{\epsilon_{n}}, \frac{r}{\epsilon_{n}}}} x_{1}\left|\Delta u_{n}\right|^{2} d x=\int_{\Theta_{n}} x_{1}\left|\Delta u_{n}\right|^{2} d x+\int_{\Sigma_{n}} x_{1}\left|\Delta u_{n}\right|^{2} d x .
$$

Como

$$
\int_{\Sigma_{n}} x_{1}\left|\Delta u_{n}\right|^{2} d x \leq \frac{R}{\epsilon_{n}} \int_{\Sigma_{n}}\left|\Delta u_{n}\right|^{2} d x
$$

e

$$
\int_{\Theta_{n}} x_{1}\left|\Delta u_{n}\right|^{2} d x \leq-\frac{r}{2 \epsilon_{n}}\left(M+o_{n}(1)\right),
$$

segue então que

$$
\int_{\Sigma_{n}}\left|\Delta u_{n}\right|^{2} d x \geq \frac{M r}{2 R}-o_{n}(1)
$$

o que contraria (3.12).

Usando o lema anterior, vamos demonstrar o seguinte resultado.

Lema 3.3.5. Existe $\epsilon^{*}>0$ tal que

$$
c(\epsilon, \Omega)<\alpha(R, r, \epsilon),
$$

para todo $\epsilon \in\left(0, \epsilon^{*}\right)$.

Demonstração. Pelo Lema 3.3.4, dado $\delta>0$, existe $\epsilon_{1}>0$ tal que

$$
\alpha(R, r, \epsilon)>c_{\infty}+\delta \quad \text { sempre que } \epsilon \in\left(0, \epsilon_{1}\right) .
$$

Usando funções corte em $B_{r}(0)$ e a função $\Psi \in H^{2}\left(\mathbb{R}^{N}\right)$ que é tal que $I_{\infty}(\Psi)=c_{\infty}$ e $I_{\infty}^{\prime}(\Psi)=0$, é possível provar que

$$
\lim _{\epsilon \rightarrow 0} c\left(\epsilon, B_{r}(0)\right)=c_{\infty}
$$

Assim, existe $\epsilon_{2}>0$ tal que

$$
c\left(\epsilon, B_{r}(0)\right)<c_{\infty}+\frac{\delta}{2} \quad \text { sempre que } \epsilon \in\left(0, \epsilon_{2}\right) .
$$

De (3.13) e (3.14), considerando $\epsilon^{*}<\min \left\{\epsilon_{1}, \epsilon_{2}\right\}$, temos que

$$
c\left(\epsilon, B_{r}(0)\right)<\alpha(R, r, \epsilon) \quad \text { sempre que } \epsilon \in\left(0, \epsilon^{*}\right) .
$$

O resultado segue então lembrando que $c(\epsilon, \Omega) \leq c\left(\epsilon, B_{r}(0)\right)$. 
O próximo resultado será essencial para que possamos construir as aplicações entre os fechados de $H^{2}\left(\mathbb{R}^{N}\right)$ e $\mathbb{R}^{N}$. Antes disso, vamos introduzir algumas notações. Sejam $\Omega_{+}$e $\Omega_{-}$ os seguintes conjuntos,

$$
\Omega_{+}=\left\{x \in \mathbb{R}^{N} ; \quad \operatorname{dist}(x, \bar{\Omega}) \leq r\right\}
$$

e

$$
\Omega_{-}=\{x \in \Omega ; \quad \operatorname{dist}(x, \partial \Omega) \geq r\} .
$$

Observe então que $\Omega_{+}, \Omega_{-}$são fechados homotopicamente equivalentes a $\bar{\Omega}$.

Lema 3.3.6. Seja $\epsilon^{*}>0$ dado como no Lema 3.3.5. Então para todo $\epsilon \in\left(0, \epsilon^{*}\right)$, existe $\Lambda^{*}=\Lambda^{*}(\epsilon)$, tal que se $\lambda>\Lambda^{*}, v \in \mathcal{N}_{\lambda, \epsilon}$ e $I_{\lambda, \epsilon}(v) \leq c\left(\epsilon, B_{r}\right)$, então $\beta_{\epsilon}(v) \in \frac{1}{\epsilon} \Omega_{+}$.

Demonstração. Suponhamos por contradição que exista uma sequência $\lambda_{n} \rightarrow \infty$ e $\left(v_{n}\right) \subset$ $H^{2}\left(\mathbb{R}^{N}\right)$ tais que $v_{n} \in \mathcal{N}_{\lambda_{n}, \epsilon}, I_{\lambda_{n}, \epsilon}\left(v_{n}\right) \leq c\left(\epsilon, B_{r}\right)$ e no entanto

$$
\beta_{\epsilon}\left(v_{n}\right) \notin \frac{1}{\epsilon} \Omega_{+} .
$$

Usando argumentos semelhantes aos empregados nas demonstrações do Lema 3.2.3 e da Proposição 3.2.7, prova-se que para cada $\eta>0$, existe $R>0$ tal que

$$
\limsup _{n \rightarrow \infty} \int_{\mathbb{R}^{N} \backslash B_{R}(0)}\left|v_{n}\right|^{2} d x<\eta \text {. }
$$

Prova-se ainda que $\left(\left\|v_{n}\right\|_{H^{2}\left(\mathbb{R}^{N}\right)}\right)$ é limitada em $\mathbb{R}$, de maneira que existe $v$ tal que $v_{n} \rightarrow v$ em $H^{2}\left(\mathbb{R}^{N}\right)$ e ainda $v=0$ em quase todo ponto de $\mathbb{R}^{N} \backslash \Omega_{\epsilon}$. Esses dois fatos implicam que $v_{n} \rightarrow v$ em $L^{2}\left(\mathbb{R}^{N}\right)$.

Como $v_{n} \in \mathcal{N}_{\lambda_{n}, \epsilon}$, pelo Lema 3.3.1 existe $\delta>0$ tal que

$$
\delta \leq\left\|v_{n}\right\|_{H^{2}\left(\mathbb{R}^{N}\right)}^{2} \leq\left\|v_{n}\right\|_{\lambda_{n}, \epsilon}^{2}=\int_{\mathbb{R}^{N}} f\left(v_{n}\right) v_{n} d x,
$$

para todo $n \in \mathbb{N}$. Disso segue que

$$
0<\delta \leq \limsup _{n \rightarrow \infty}\left\|v_{n}\right\|_{\lambda_{n}, \epsilon}^{2}=\int_{\mathbb{R}^{N}} f(v) v d x
$$

Assim, $v \neq 0$ em $H_{0}^{2}\left(\Omega_{\epsilon}\right)$ e $\lim _{n \rightarrow \infty} \beta_{\epsilon}\left(v_{n}\right)=\beta(u)$. Dessa forma, por (3.15), segue que $y=\beta(u) \notin \frac{1}{\epsilon} \Omega_{+}$. Para esse $y$, existe um $R>0$ grande o suficiente de tal forma que $\Omega_{\epsilon} \subset A_{\frac{R}{\epsilon}, \frac{r}{\epsilon}, y}$. Assim, como $v \neq 0$ em $H_{0}^{2}\left(\Omega_{\epsilon}\right)$, segue que sua estensão por 0 fora de $\Omega_{\epsilon}$ é não nula em $H_{0}^{2}\left(A_{\frac{R}{\epsilon}, \frac{r}{\epsilon}, y}\right)$. Dessa forma, existe $t>0$ tal que $t u \in \hat{\mathcal{N}}_{\epsilon, y}$. Assim

$$
\hat{I}_{\epsilon, y}(t u)=I_{\epsilon}(t u) \leq \liminf _{n \rightarrow \infty} I_{\lambda_{n}, \epsilon}\left(t v_{n}\right) \leq \liminf _{n \rightarrow \infty} I_{\lambda_{n}, \epsilon}\left(v_{n}\right) \leq c\left(\epsilon, B_{r}\right) .
$$

Como $\beta(t u)=\beta(u)$, temos que $\alpha(R, r, \epsilon, y) \leq c\left(\epsilon, B_{r}\right)$.

Por outro lado, como $\alpha(R, r, \epsilon, y)=\alpha(R, r, \epsilon)$, temos que $\alpha(R, r, \epsilon) \leq c\left(\epsilon, B_{r}\right)$, o que contraria o final da demonstração do Lema 3.3.5. 


\subsection{Demonstração do Teorema 3.1.1}

Vamos continuar considerando $r>0$ tal que $B_{r}(0) \subset \Omega$ e seja $\epsilon^{*}>0$ dado pelo Lema 3.3.6. Para $\epsilon \in\left(0, \epsilon^{*}\right)$, consideremos o seguinte conjunto

$$
I_{\lambda, \epsilon}^{c\left(\epsilon, B_{r}\right)}=\left\{v \in \mathcal{N}_{\lambda, \epsilon} ; \quad I_{\lambda, \epsilon}(v) \leq c\left(\epsilon, B_{r}\right)\right\} .
$$

Queremos provar que

$$
\operatorname{cat}_{\lambda, \epsilon}^{c\left(\epsilon, B_{r}\right)} \geq \operatorname{cat}(\bar{\Omega}), \quad \forall \epsilon \in\left(0, \epsilon^{*}\right) \text { e } \lambda>\Lambda^{*},
$$

onde $\Lambda^{*}$ é dado pelo Lema 3.3.6. Como $\frac{1}{\epsilon} \Omega_{+}, \frac{1}{\epsilon} \Omega_{-}$e $\bar{\Omega}$ são subconjuntos homotopicamente equivalentes, então

$$
\operatorname{cat}(\bar{\Omega})=\operatorname{cat}_{\frac{1}{\epsilon} \Omega_{+}}\left(\frac{1}{\epsilon} \Omega_{-}\right) .
$$

Afirmação 3.4.1. Para toda cobertura $\left\{\mathcal{O}_{i}, i=1, \ldots, n\right\}$ de $I_{\lambda, \epsilon}^{c\left(\epsilon, B_{r}\right)}$ por fechados contráteis a um ponto de $I_{\lambda, \epsilon}^{c\left(\epsilon, B_{r}\right)}$, existe uma cobertura de $\frac{1}{\epsilon} \Omega_{-}$por fechados $\mathcal{B}_{i}, i=1, \ldots, n$ contráteis a um ponto de $\frac{1}{\epsilon} \Omega_{+}$.

Note que a seguinte afirmação implica

$$
\operatorname{cat}_{\frac{1}{\epsilon} \Omega_{+}}\left(\frac{1}{\epsilon} \Omega_{-}\right) \leq \operatorname{cat}\left(I_{\lambda, \epsilon}^{c\left(\epsilon, B_{r}\right)}\right) .
$$

Supondo que a afirmação valha, por (3.17) e (3.18) vale a desigualdade (3.16). Assim, uma vez que pela Proposição 3.2 .1 o funcional $I_{\lambda, \epsilon}$ satisfaz $(P S)_{c}$ para todo $c \leq c\left(\epsilon, B_{r}\right)$ contanto que $\lambda \geq \Lambda^{*}$, segue do Teorema 3.3 .3 e de (3.16) que $I_{\lambda, \epsilon}$ possui pelo menos $\operatorname{cat}(\bar{\Omega})$ pontos críticos não-triviais em $I_{\lambda, \epsilon}^{c\left(\epsilon, B_{r}\right)}$

Portanto, para terminar a demonstração do Teorema 3.1.1 é suficiente provarmos a Afirmação 3.4.1

Demonstração da Afirmação 3.4.1. Seja $I_{\lambda, \epsilon}^{c\left(\epsilon, B_{r}\right)}=\bigcup_{i=1}^{n} \mathcal{O}_{i}$, onde $\mathcal{O}_{i}, i=1, \ldots, n$, são subconjuntos fechados e contráteis a um ponto em $I_{\lambda, \epsilon}^{c\left(\epsilon, B_{r}\right)}$, ou seja, existem $n$ aplicações $h_{i} \in C\left([0,1] \times \mathcal{O}_{i}, I_{\lambda, \epsilon}^{c\left(\epsilon, B_{r}\right)}\right), i=1, \ldots, n$, tais que para todo $v \in \mathcal{O}_{i}, h_{i}(0, v)=v$ e $h_{i}(1, v)=w_{i}$ para algum $w_{i} \in I_{\lambda, \epsilon}^{c\left(\epsilon, B_{r}\right)}$.

Para construir a cobertura fechada $\left\{\mathcal{B}_{i}, i=1, \ldots, n\right\}$ de $\frac{1}{\epsilon} \Omega_{-}$, consideremos $v_{r \epsilon} \in H_{0}^{2}\left(B_{r}(0)\right)$ o ponto crítico de $I_{\epsilon, B_{r}}$ dado pelo Teorema do Passo da Montanha. Pela hipótese $\left(f_{5}\right)$, sabemos que $I_{\epsilon, B_{r}}\left(v_{r \epsilon}\right)=c\left(\epsilon, B_{r}\right)$ e pelo Teorema 7.1 de [21], segue que $v_{r \epsilon}$ é radialmente simétrica com relação a 0 . Definamos agora a função $\Psi_{r}: \frac{1}{\epsilon} \Omega_{-} \rightarrow H_{0}^{2}\left(\Omega_{\epsilon}\right)$, por

$$
\Psi_{r}(y)(x)=\left\{\begin{array}{l}
v_{r \epsilon}(|x-y|), \\
0, \text { se } x \notin B_{\frac{r}{\epsilon}}(y) .
\end{array} \quad \text { se } x \in B_{\frac{r}{\epsilon}}(y)\right.
$$

Uma vez que $v_{r \epsilon}$ é radialmente simétrico com relação a origem, segue que $\beta_{\epsilon}\left(\Psi_{r}(y)\right)=y$, para todo $y \in \frac{1}{\epsilon} \Omega_{-}$. 
Seja agora $\mathcal{B}_{i}=\beta_{\epsilon}\left(\mathcal{O}_{i}\right), i=1, \ldots, n$. Observe que $\frac{1}{\epsilon} \Omega_{-}=\bigcup_{i=1}^{n} \mathcal{B}_{i}$. De fato, seja $y \in \frac{1}{\epsilon} \Omega_{-}$. Note que $\Psi_{r}(y)$ é uma translação por $y$ de $u_{r \epsilon}$, de forma que, como $y \in \frac{1}{\epsilon} \Omega_{-}$, $\operatorname{supp}\left(\Psi_{r}(y)\right) \subset B_{\frac{r}{\epsilon}}(y) \subset \Omega_{\epsilon}$. Assim

$$
I_{\lambda, \epsilon}^{\prime}\left(\Psi_{r}(y)\right) \Psi_{r}(y)=I_{\epsilon, B_{r}}^{\prime}\left(u_{r \epsilon}\right) u_{r \epsilon}=0 \quad \text { e } \quad I_{\lambda, \epsilon}\left(\Psi_{r}(y)\right)=I_{\epsilon, B_{r}}\left(u_{r \epsilon}\right)=c\left(\epsilon, B_{r}\right),
$$

de forma que $\Psi_{r}(y) \in I_{\lambda, \epsilon}^{c\left(\epsilon, B_{r}\right)}$. Portanto $\Psi_{r}(y) \in \mathcal{O}_{i}$, para algum $i \in\{1, \ldots, n\}$. Logo $y \in \mathcal{B}_{i}$.

Para verificar que para todo $i \in\{1, \ldots, n\}, \mathcal{B}_{i}$ é contrátil a um ponto de $\frac{1}{\epsilon} \Omega_{+}$, consideremos a função $\gamma_{i}:[0,1] \times \mathcal{B} i \rightarrow \mathbb{R}^{N}$ dada por

$$
\gamma_{i}(t, y)=\beta_{\epsilon}\left(h_{i}\left(t, \Psi_{r}(y)\right)\right)
$$

Note que a função $\gamma_{i}$ satisfaz, para todo $y \in \mathcal{B}_{i}$,

$$
\begin{aligned}
& \gamma_{i}(0, y)=\beta_{\epsilon}\left(\Psi_{r}(y)\right)=y \\
& \gamma_{i}(1, y)=\beta_{\epsilon}\left(w_{i}\right)
\end{aligned}
$$

Porém, como para todo $y \in \mathcal{B}_{i}, \Psi_{r}(y) \in \mathcal{O}_{i} \subset I_{\lambda, \epsilon}^{c\left(\epsilon, B_{r}\right)}$, pelo Lema 3.3 .6 temos que $\gamma_{i}(t, y)=\beta_{\epsilon}\left(h_{i}\left(t, \Psi_{r}(y)\right)\right) \in \frac{1}{\epsilon} \Omega_{+}$, para todo $t \in[0,1]$. Assim $\gamma_{i}$ é uma deformação de $\mathcal{B}_{i}$ a um ponto de $\frac{1}{\epsilon} \Omega_{+}$.

Isso encerra a demonstração da afirmação. 


\section{4}

\section{Existência de soluções nodais para uma equação biharmônica}

Nesse capítulo, seguindo os argumentos de Weth em [36], vamos estudar a seguinte equação biharmônica com condição de fronteira do tipo Navier,

$$
\left\{\begin{aligned}
\Delta^{2} u & =f(x, u) \text { em } \Omega \\
u & =\Delta u=0 \text { em } \partial \Omega,
\end{aligned}\right.
$$

onde $N \geq 5, \Omega \subset \mathbb{R}^{N}$ é um domínio aberto, limitado e com fronteira suave e $f: \Omega \times \mathbb{R} \rightarrow \mathbb{R}$ satisfaz as seguintes hipóteses:

$\left(f_{1}\right) f$ é uma função de Carathéodory e $f(x, 0)=0$, para quase todo $x \in \Omega$;

$\left(f_{2}\right)$ existem $c_{1} \in\left(0, \lambda_{1}\right), c_{2}>0$ e $0<p<2_{*}-2$ tais que

$$
|f(x, t)-f(x, s)| \leq\left(c_{1}+c_{2}\left(|t|^{p}+|s|^{p}\right)\right)|t-s|
$$

para quase todo $x \in \Omega$ e $s, t \in \mathbb{R}$;

$\left(f_{3}\right)$

$$
\lim _{|s| \rightarrow \infty} \frac{F(x, s)}{s^{2}}=+\infty,
$$

uniformemente para quase todo $x \in \Omega$; 
$\left(f_{4}\right)$ existe $C>0$ tal que $H(x, t) \leq H(x, s)+C$, para $0<t<s$ ou $s<t<0$ e para quase todo $x$ em $\Omega$, onde $H(x, t)=f(x, t) t-2 F(x, t)$.

$\left(f_{5}\right) f(x, t)$ é não-decrescente para $t \in \mathbb{R}$ e quase todo $x \in \Omega$.

Observação 4.0.2. Na hipótese $\left(f_{2}\right), \lambda_{1}$ denota o primeiro autovalor do operador $\left(\Delta^{2}, H^{2} \cap H_{0}^{1}(\Omega)\right)$.

Desejamos provar o seguinte resultado

Teorema 4.0.3. Se $f$ satisfaz $\left(f_{1}\right)-\left(f_{5}\right)$, então (4.1) possui ao menos três soluções fracas não-triviais, sendo uma não-negativa, uma não-positiva e uma nodal.

A demonstração desse teorema seguirá de argumentos variacionais e da análise do fluxo de uma determinada EDO. Para essa análise, será de fundamental importância estudarmos o método de decomposição em cones duais, introduzido por Moreau em [28], o que é feito na primeira seção desse capítulo. A nossa contribuição para o estudo do problema (4.1), é apresentar o mesmo resultado de Weth em [36], porém com uma demonstração que não requer a condição de Ambrosetti-Rabinowitz. Essa, por sua vez, foi substituída pela condição mais fraca de superquadraticidade $\left(f_{3}\right)$ e também por $\left(f_{4}\right)$. Como veremos, as condições $\left(f_{3}\right)$ e $\left(f_{4}\right)$ serão suficientes para mostrar que o fluxo é uniformemente limitado, garantindo que o conjunto $\omega$-limite é não vazio (ver Proposição 4.2.2).

\subsection{Método de decomposição em cones duais}

Seja $(H,\langle\cdot, \cdot\rangle)$ um espaço de Hilbert.

Definição 4.1.1. - Um conjunto $\mathcal{K} \subset H$ é chamado de cone se para todo $x \in \mathcal{K}$ e $\lambda \geq 0$, $\lambda x \in \mathcal{K}$.

- Dado $\mathcal{K}$ um cone, definimos seu cone dual por

$$
\mathcal{K}^{*}=\{v \in H ; \quad\langle u, v\rangle \leq 0, \quad \forall u \in \mathcal{K}\}
$$

O próximo resultado mostra que, dado um cone convexo e fechado $\mathcal{K}$, sempre é possível decompor um vetor de $H$ como uma soma de um elemento de $\mathcal{K}$ e um de $\mathcal{K}^{*}$.

Teorema 4.1.2. Seja $\mathcal{K} \subset H$ um cone convexo e fechado. Todo $x \in H$ pode ser decomposto na forma

$$
x=y+z \text { onde } y \in \mathcal{K} \text { e } z \in \mathcal{K}^{*}
$$

e ainda

$$
\langle y, z\rangle=0
$$


Demonstração. Se $x \in H$ e $A \subset H$ é um convexo fechado, denotemos por $\operatorname{proj}_{A} x=$ $\inf _{y \in A}\|x-y\|$ a projeção ortogonal de $x$ sobre $A$. Lembre-se ainda que a projeção satisfaz

$$
\left\langle x-\operatorname{proj}_{A} x, p-\operatorname{proj}_{A} x\right\rangle \leq 0, \quad \forall p \in A .
$$

Seja então $y=\operatorname{proj}_{\mathcal{K}} x$ e $z=x-y$. Temos então que

$$
\langle x-y, p-y\rangle \leq 0 \quad \forall p \in \mathcal{K} .
$$

Escolhendo $p=\lambda y$ (o que é possível pois $\mathcal{K}$ é um cone), onde $\lambda \geq 0$ é arbitrário, temos

$$
\langle x-y, \lambda y-y\rangle=(\lambda-1)\langle z, y\rangle \leq 0, \quad \forall \lambda \geq 0 .
$$

$\operatorname{Logo}\langle z, y\rangle=0$. Assim

$$
\langle z, p\rangle=\langle z, p-y\rangle \leq 0, \quad \forall p \in \mathcal{K},
$$

ou seja, $z \in \mathcal{K}^{*}$.

Alguns exemplos interessantes desta decomposição podem ser encontrados em [15].

\subsection{Argumentos variacionais}

Seja $H=H^{2} \cap H_{0}^{1}(\Omega)$ e consideremos o seguinte produto interno

$$
\langle u, v\rangle=\int_{\Omega} \Delta u \Delta v d x,
$$

que dá origem a uma norma $\|\cdot\|$. Observe que $(H,\langle\cdot, \cdot\rangle)$ é um espaço de Hilbert.

Consideremos agora o funcional energia $I: H \rightarrow \mathbb{R}$ associado a (4.1) e o operador $A: H \rightarrow H$, dados por

$$
I(u)=\frac{\|u\|^{2}}{2}-\int_{\Omega} F(x, u) d x
$$

e

$$
A(u)=\left(\Delta^{2}\right)^{-1} f(\cdot, u(\cdot)) .
$$

Lema 4.2.1. O operador A está bem definido e é caracterizado por

$$
\langle A(u), v\rangle=\int_{\Omega} f(x, u) v d x, \quad u, v \in H .
$$

Além disso,

$\left(A_{1}\right) \quad A=\nabla \Psi$, onde $\Psi(u)=\int_{\Omega} F(x, u) d x$ e existem $d_{1} \in(0,1)$ e $d_{2}>0$ tais que

$$
|\langle A(u), v\rangle| \leq\left(d_{1}\|u\|+d_{2}\|u\|^{p+1}\right)\|v\|, \quad u, v \in H ;
$$


$\left(A_{2}\right)$ A é compacto e localmente lipschitziano. Além disso, $A(0)=0$.

Demonstração. Mostremos primeiramente que o operador $A$ está bem definido. Para isso usaremos estimativas $L^{p}$ para operadores elípticos de ordem mais alta. Observe que por $\left(f_{2}\right)$, se $u \in H$, então $f(\cdot, u(\cdot)) \in L^{\frac{2 *}{p+1}}(\Omega)$. Pela estimativa $L^{p}$ constante no Corolário 2.21 de [21], $\left(\Delta^{2}\right)^{-1} f(\cdot, u(\cdot)) \in W^{4, \frac{2 *}{p+1}} \cap W_{0}^{2, \frac{2 *}{p+1}}(\Omega)$. Uma vez que $\Omega$ é limitado e $p+1<\frac{N+4}{N-4}$, temos a imersão contínua $W^{4, \frac{2 *}{p+1}}(\Omega) \hookrightarrow W^{2,2}(\Omega)$. Logo está bem definido o operador

$$
\left(\Delta^{2}\right)^{-1} f: H \rightarrow H
$$

Note que, para todo $u, v \in H$,

$$
\begin{aligned}
\langle A(u), v\rangle & =\int_{\Omega} \Delta\left(\left(\Delta^{2}\right)^{-1} f(x, u(x)) \Delta v(x) d x\right. \\
& =\int_{\Omega} \Delta^{2}\left(\left(\Delta^{2}\right)^{-1} f(x, u(x)) v(x) d x\right. \\
& =\int_{\Omega} f(x, u(x)) v(x) d x .
\end{aligned}
$$

Para provar que $A=\nabla \Psi$, basta usar a definição de diferenciabilidade de $\Psi$ e o Teorema da Convergência Dominada Generalizado.

A estimativa em $\left(A_{1}\right)$ segue de $\left(f_{2}\right)$ da seguinte forma

$$
\begin{aligned}
|\langle A(u), v\rangle| & \leq \int_{\Omega}|f(x, u) \| v| d x \\
& \leq \int_{\Omega}\left(c_{1}|u \| v|+c_{2}|u|^{p+1}|v|\right) d x .
\end{aligned}
$$

Usando a Desigualdade de Hölder com os expoentes $\frac{2_{*}}{p+1}$ e $\frac{2_{*}}{2_{*}-(p+1)}$, temos que

$$
\begin{aligned}
\int_{\Omega}\left(c_{1}|u||v|+c_{2}|u|^{p+1}|v|\right) d x & \leq c_{1}\|u\|_{L^{2}}\|v\|_{L^{2}}+c_{2}\|u\|_{L^{2 *}}^{p+1}\|v\|_{L^{\frac{2_{*}}{2 *-(p+1)}}} \\
& \leq c_{1}\|u\|_{L^{2}}\|v\|_{L^{2}}+C c_{2}\|u\|^{p+1}\|v\| \\
& \leq \frac{c_{1}}{\lambda_{1}}\|u\|\|v\|+C c_{2}\|u\|^{p+1}\|v\|,
\end{aligned}
$$

onde usamos a caracterização variacional de $\lambda_{1}$. Note que $d_{1}:=\frac{c_{1}}{\lambda_{1}} \in(0,1), d_{2}:=C c_{2}>0$ e a estimativa segue.

Considerando agora $\left(A_{2}\right)$, note que $A(0)=0$. A compacidade de $A$ segue de $\left(f_{2}\right)$, juntamente com a compacidade das imersões de Sobolev $H^{2}(\Omega) \hookrightarrow L^{q}(\Omega)$, para $1 \leq q<2_{*}$.

Mostremos finalmente que $A$ é localmente lipschitziano.

Sejam $u, v \in B_{r}(0)$ em $H$. Observe primeiramente que o operador $\left(\delta^{2}\right)^{-1} f$ está bem definido de $H$ em $L^{1}(\Omega)$, devido às imersões de Sobolev e além disso é contínuo. Na estimativa que se segue, $C$ denotará uma constante positiva que pode depender de $\Omega$ e $r$, mas nunca de 
$u$ ou $v$.

$$
\begin{aligned}
\|A(u)-A(v)\| & =\left\|\left(\Delta^{2}\right)^{-1}(f(\cdot, u)-f(\cdot, v))\right\| \\
& \leq C\|f(\cdot, u)-f(\cdot, v)\|_{L^{1}(\Omega)} \\
& =C \int_{\Omega}|f(\cdot, u)-f(\cdot, v)| d x \\
& \leq C \int_{\Omega}\left(c_{1}|u-v|+c_{2}\left(|u|^{p}+|v|^{p}\right)|u-v|\right) d x .
\end{aligned}
$$

Usando-se Hölder com os expoentes conjugados $\frac{2_{*}}{p}$ e $\frac{2_{*}}{2_{*}-p}$ e mais a imersão contínua de $L^{1}(\Omega)$ em $L^{2}(\Omega)$, temos que

$$
\begin{aligned}
\|A(u)-A(v)\| & \leq C\|u-v\|_{L^{2}(\Omega)}+C\|u\|_{L^{2 *}(\Omega)}^{p}\|u-v\|_{L^{\frac{2 *}{2 *}-p}}+C\|v\|_{L^{2 *}(\Omega)}^{p}\|u-v\|_{L^{2 *}-p} \\
& \leq C\|u-v\|+C\|u-v\|_{L^{2 *}} \\
& \leq C\|u-v\|,
\end{aligned}
$$

o que encerra a demonstração.

Para justificar o emprego de argumentos variacionais, vamos obter as soluções fracas de (4.1) como pontos críticos do funcional energia $I$. Esses por sua vez são exatamente os pontos fixos do operador $A$, como pode ser facilmente verificado. Para a obtenção desses pontos críticos vamos estudar a dinâmica da seguinte EDO:

$$
\left\{\begin{aligned}
\frac{\partial}{\partial t} \varphi(t, u) & =-\nabla I(\varphi(t, u))=A(\varphi(t, u))-\varphi(t, u), \\
\varphi(0, u) & =u,
\end{aligned}\right.
$$

onde $\varphi: \mathcal{G} \rightarrow H, \mathcal{G}=\{(t, u) ; u \in H, 0 \leq t<T(u)\}$ e $[0, T(u))$ é o intervalo maximal de existência da trajetória $t \mapsto \varphi(t, u)$. Observe que o fluxo está bem definido uma vez que $A$ é localmente lipschitziano.

No próximo resultado, a falta da condição de Ambrosetti-Rabinowitz representa uma grande dificuldade. Com efeito, em [36], como o autor dispunha da condição $(A R)$, facilmente provou-se que o funcional $I$ satisfaz a condição de Palais-Smale. Isso tornava a demonstração do próximo resultado trivial. Nós porém, precisamos utilizar argumentos alternativos que foram adaptados de [27].

Proposição 4.2.2. Se para algum $u \in H,\{I(\varphi(t, u)) ; 0 \leq t<T(u)\}$ for limitado inferiormente, então

i) $T(u)=\infty$,

ii) existe $t_{n} \rightarrow \infty$ tal que $\left\{\varphi\left(t_{n}, u\right), n \in \mathbb{N}\right\}$ é limitado em $H$ e o conjunto ômega limite de $u$

$$
\omega(u)=\bigcap_{0 \leq t<\infty} \overline{\bigcup_{t \leq s<\infty} \varphi(s, u)}
$$

é não-vazio e formado por pontos críticos de $I$. 
Demonstração. i) O resultado seguirá da seguinte estimativa

$$
\begin{aligned}
\|\varphi(t, u)-\varphi(s, u)\| & \leq \int_{s}^{t}\|\nabla I(\varphi(\tau, u))\| d \tau \\
& \leq \sqrt{t-s}\left(\int_{s}^{t}\|\nabla I(\varphi(\tau, u))\|^{2} d \tau\right)^{\frac{1}{2}} \\
& =\sqrt{t-s}(I(\varphi(s, u))-I(\varphi(t, u)))^{\frac{1}{2}}
\end{aligned}
$$

onde foi usado a Desigualdade de Hölder e a própria equação (4.2). Supondo que $T(u)<\infty$, então a trajetória seria limitada. Dessa forma a teoria das EDO's nos garantiria que $T(u)=\infty$, o que seria uma contradição.

ii) Observemos primeiramente que existe uma sequência $t_{n} \rightarrow \infty$ tal que $\left\|\nabla I\left(\varphi\left(t_{n}, u\right)\right)\right\| \rightarrow 0$, quando $n \rightarrow \infty$. De fato, note que

$$
\begin{aligned}
\int_{0}^{\infty}\|\nabla I(\varphi(\tau, u))\|^{2} d \tau & =\lim _{t \rightarrow \infty} \int_{0}^{t}\|\nabla I(\varphi(\tau, u))\|^{2} d \tau \\
& =\lim _{t \rightarrow \infty}|I(\varphi(t, u))-I(u)|<\infty
\end{aligned}
$$

de forma que existe $t_{n} \rightarrow \infty$ tal que $\left\|\nabla I\left(\varphi\left(t_{n}, u\right)\right)\right\|^{2} \rightarrow 0$.

Afirmamos que $\left\{\varphi\left(t_{n}, u\right)\right\}_{n \in \mathbb{N}}$ é limitada uniformemente em $H$ com respeito a $n \in \mathbb{N}$. De fato, suponhamos por contradição que $\left\|\varphi\left(t_{n}, u\right)\right\| \rightarrow \infty$, quando $n \rightarrow \infty$. Definamos então

$$
w_{n}=\frac{\varphi\left(t_{n}, u\right)}{\left\|\varphi\left(t_{n}, u\right)\right\|} .
$$

Sendo $\left(w_{n}\right)$ uma sequência limitada em $H$, sabemos então que existe $w \in H$ tal que, a menos de subsequência $w_{n} \rightarrow w$ em $H$ e, consequentemente, $w_{n} \rightarrow w$ em $L^{q}(\Omega)$, $1<q<2 *$ e também em quase todo ponto de $\Omega$.

Mostremos que $w=0$. Seja $\Gamma=\{x \in \Omega ; w(x) \neq 0\}$, mostremos que $\Gamma$ tem medida nula. Note que para quase todo $x \in \Gamma$, temos necessariamente que $\lim _{n \rightarrow \infty} \varphi\left(t_{n}, u\right)(x)=\infty$. Por $\left(f_{3}\right)$, dado $M>0$, existem $K_{M}>0$ e $C_{M}>0$, tais que

$$
F(x, s) \geq M s^{2}-C_{M}, \quad \text { para todo } s \in \mathbb{R} \text { e para todo } x \in \Omega \text {. }
$$

Seja $n_{0} \in \mathbb{N}$ tal que $\varphi\left(t_{n}, u\right)(x) \geq K_{M}$, para quase todo $x \in \Gamma$. Então, para $n \geq n_{0}$, temos que

$$
\begin{aligned}
\int_{\Omega} \frac{F\left(x, \varphi\left(t_{n}, u\right)\right)}{\varphi\left(t_{n}, u\right)^{2}} w_{n}^{2} & =\int_{\Gamma} \frac{F\left(x, \varphi\left(t_{n}, u\right)\right)}{\varphi\left(t_{n}, u\right)^{2}} w_{n}^{2}+\int_{\Omega \backslash \Gamma} \frac{F\left(x, \varphi\left(t_{n}, u\right)\right)}{\varphi\left(t_{n}, u\right)^{2}} w_{n}^{2} \\
& \geq M \int_{\Gamma} w_{n}^{2} d x+M \int_{\Omega \backslash \Gamma} w_{n}^{2} d x-\frac{C_{M}|\Omega \backslash \Gamma|}{\left\|\varphi\left(t_{n}, u\right)\right\|^{2}} \\
& =M \int_{\Gamma} w_{n}^{2} d x+o_{n}(1) .
\end{aligned}
$$


Usando que $\left(I\left(\varphi\left(t_{n}, u\right)\right)\right)$ é limitado inferiormente, existe $\alpha>0$ tal que

$$
-\alpha \leq I\left(\varphi\left(t_{n}, u\right)\right), \quad \text { para todo } n \in \mathbb{N} .
$$

Dividindo (4.3) por $\left\|\varphi\left(t_{n}, u\right)\right\|^{2}$, temos que

$$
\int_{\Omega} \frac{F\left(x, \varphi\left(t_{n}, u\right)\right)}{\left\|\varphi\left(t_{n}, u\right)\right\|^{2}} d x \leq \frac{1}{2}+O_{n}(1) .
$$

Então,

$$
M \int_{\Gamma} w_{n}^{2} d x \leq \frac{1}{2}+o_{n}(1)
$$

Fazendo $n \rightarrow+\infty$, obtemos

$$
M \int_{\Gamma} w^{2} d x \leq \frac{1}{2}
$$

o que é impossível se $|\Gamma|>0$, pois $M>0$ é arbitrário e $w \neq 0$ em quase todo ponto de $\Gamma$. Portanto, $|\Gamma|=0$.

Note agora que a função $t \mapsto I\left(t \varphi\left(t_{n}, u\right)\right)$ é diferenciável em $(0,1)$. Seja $s_{n} \in[0,1]$ tal que

$$
I\left(s_{n} \varphi\left(t_{n}, u\right)\right)=\max _{t \in[0,1]} I\left(t \varphi\left(t_{n}, u\right)\right) .
$$

Observe que, dado $R>0$, para todo $n$ suficientemente grande,

$$
\begin{aligned}
I\left(s_{n} \varphi\left(t_{n}, u\right)\right) & \geq I\left(\frac{R}{\left\|\varphi\left(t_{n}, u\right)\right\|} \varphi\left(t_{n}, u\right)\right) \\
& =\frac{R^{2}}{2}-\int_{\Omega} F\left(x, R w_{n}\right) d x=\frac{R^{2}}{2}+o_{n}(1) .
\end{aligned}
$$

Assim $\lim _{n \rightarrow \infty} I\left(s_{n} \varphi\left(t_{n}, u\right)\right)=+\infty$, o que implica $s_{n} \in(0,1)$. Portanto $I^{\prime}\left(s_{n} \varphi\left(t_{n}, u\right)\right) s_{n} \varphi\left(t_{n}, u\right)=0$. Dessa forma, usando $\left(f_{4}\right)$, para todo $t \in[0,1]$ temos que

$$
\begin{aligned}
2 I\left(t \varphi\left(t_{n}, u\right)\right) & \leq 2 I\left(s_{n} \varphi\left(t_{n}, u\right)\right)-I^{\prime}\left(s_{n} \varphi\left(t_{n}, u\right)\right) s_{n} \varphi\left(t_{n}, u\right) \\
& =\int_{\Omega}\left(f\left(x, s_{n} \varphi\left(t_{n}, u\right)\right) s_{n} \varphi\left(t_{n}, u\right)-2 F\left(x, s_{n} \varphi\left(t_{n}, u\right)\right)\right) d x \\
& \leq \int_{\Omega}\left(f\left(x, \varphi\left(t_{n}, u\right)\right) \varphi\left(t_{n}, u\right)-2 F\left(x, \varphi\left(t_{n}, u\right)\right)+C\right) d x \\
& =2 I\left(\varphi\left(t_{n}, u\right)\right)+C|\Omega| \leq C_{1}
\end{aligned}
$$

Note que, dado $R_{0}>0$ qualquer, para todo $n$ suficientemente grande, $\frac{R_{0}}{\left\|\varphi\left(t_{n}, u\right)\right\|}<1$. Assim, pela estimativa acima

$$
2 I\left(R_{0} w_{n}\right)=2 I\left(\frac{R_{0}}{\left\|\varphi\left(t_{n}, u\right)\right\|} \varphi\left(t_{n}, u\right)\right) \leq C_{1} .
$$

Por outro lado, para todo $R_{0}>0$,

$$
2 I\left(R_{0} w_{n}\right)=R_{0}^{2}-2 \int_{\Omega} F\left(x, R_{0} w_{n}\right) d x=R_{0}^{2}+o_{n}(1),
$$


onde usamos que $w_{n} \rightarrow 0$ em $L^{q}(\Omega)$ para $1<q<2_{*}$, juntamente com o Teorema da Convergência Dominada.

Observe agora que (4.5) contradiz (4.4) e essa contradição implica de fato $\left\{\varphi\left(t_{n}, u\right)\right\}_{n \in \mathbb{N}}$ é limitada uniformemente em $H$ com respeito a $n \in \mathbb{N}$ e a afirmação está verificada.

Para mostrar que $\omega(u) \neq \emptyset$, vamos usar a sequência limitada $\left\{\varphi\left(t_{n}, u\right)\right\}$. Como $A$ é compacto, segue que a menos de uma subsequência, $A\left(\varphi\left(t_{n}, u\right)\right) \rightarrow u_{0}$ quando $n \rightarrow \infty$, para algum $u_{0} \in H$. Dessa forma, note que

$$
\begin{aligned}
0 & =\lim _{n \rightarrow \infty}\left\|\nabla I\left(\varphi\left(t_{n}, u\right)\right)\right\| \\
& =\lim _{n \rightarrow \infty}\left(\varphi\left(t_{n}, u\right)-A\left(\varphi\left(t_{n}, u\right)\right)\right) \\
& =\lim _{n \rightarrow \infty} \varphi\left(t_{n}, u\right)-u_{0} .
\end{aligned}
$$

Portanto $\lim _{n \rightarrow \infty} \varphi\left(t_{n}, u\right)=u_{0}$ e $u_{0} \in \omega(u)$.

Para mostrar que todo elemento de $\omega(u)$ é ponto crítico de $I$, note primeiramente que $I(\varphi(t, u)) \rightarrow d$ quando $t \rightarrow+\infty$, pois essa é uma sequência monótona decrescente e limitada. Então, se $v \in \omega(u)$, existe $t_{n} \rightarrow \infty$ tal que $\varphi\left(t_{n}, u\right) \rightarrow v$ em $H$, quando $n \rightarrow \infty$. Assim

$$
I(\varphi(t, v))=\lim _{n \rightarrow \infty} I\left(\varphi\left(t, \varphi\left(t_{n}, u\right)\right)\right)=\lim _{n \rightarrow \infty} I\left(\varphi\left(t+t_{n}, u\right)\right)=d, \quad \text { para todo } t \geq 0 .
$$

Dessa forma, para todo $t \geq 0$

$$
0=\frac{\partial}{\partial t} I(\varphi(t, v))=\|\nabla I(\varphi(t, v))\|^{2}
$$

Aplicando para $t=0$, obtemos que $\nabla I(v)=0$ e $v$ é um ponto crítico para $I$.

Definição 4.2.3. $\quad$ - $D \subset H$ é positivamente invariante se para todo $u \in D, \varphi(t, u) \in D$, para todo $t \in[0, T(u))$;

- se $D \subset H$ é positivamente invariante, definimos seu domínio de absorção $\mathcal{A}(D)$ por

$$
\mathcal{A}(D)=\left\{u \in H ; \exists t_{0} \in[0, T(u)) \text { tal que } \varphi\left(t_{0}, u\right) \in D\right\}
$$

- $\mathcal{A}_{0}=\{u \in H ; T(u)=\infty$ e $\varphi(t, u) \rightarrow 0$ quando $t \rightarrow \infty\}$.

Lema 4.2.4. $\mathcal{A}_{0}$ é uma vizinhança aberta de $0 \mathrm{em} H$. 
Demonstração. Seja $\alpha_{0}=\left(\frac{1-c_{1}}{2 c_{2}}\right)^{\frac{1}{p}}$. Para todo $u \in \overline{B_{\alpha_{0}}(0)}$, temos por $\left(A_{1}\right)$ que

$$
\begin{aligned}
\Psi(u) & =\int_{0}^{1} \frac{\partial}{\partial t} \Psi(t u) d t \\
& =\int_{0}^{1}\langle A(t u), u\rangle d t \\
& \leq \int_{0}^{1}\left(c_{1}\|t u\|+c_{2}\|t u\|^{p+1}\right)\|u\| d t \\
& \leq \int_{0}^{1} t\|u\|^{2}\left(c_{1}+c_{2}\|u\|^{p}\right) d t \\
& =\frac{\|u\|^{2}}{2}\left(c_{1}+c_{2}\|u\|^{p}\right) \\
& \leq \frac{\|u\|^{2}}{2}\left(c_{1}+c_{2} \alpha_{0}^{p}\right) \\
& =\frac{c_{1}+1}{4}\|u\|^{2} .
\end{aligned}
$$

Assim,

$$
I(u)=\frac{1}{2}\|u\|^{2}-\Psi(u) \geq\left(\frac{1}{2}-\frac{c_{1}+1}{4}\right)\|u\|^{2} \geq 0,
$$

pois $c_{1} \in(0,1)$.

Ainda, para todo $u \in \partial B_{\alpha_{0}}(0)$,

$$
I(u) \geq\left(\frac{1}{2}-\frac{c_{1}+1}{4}\right) \alpha_{0}^{2}=: \beta_{0}>0 .
$$

Pela continuidade de $I$ e pelo fato de $I(0)=0$, existe $r \in\left(0, \alpha_{0}\right)$ tal que

$$
I(u)<\beta_{0} \quad \text { para todo } u \in B_{r}(0) .
$$

Como a energia não cresce ao longo da trajetória, se $u \in B_{r}(0)$, então $I(\varphi(t, u))<\beta_{0}$, para todo $t \in[0, T(u))$. Logo, por (4.6), $\varphi(t, u) \in B_{\alpha_{0}}(0)$ para todo $t \in[0, T(u))$. Assim, $I(\varphi(t, u)) \geq 0$, para todo $t \in[0, T(u))$, de forma que $T(u)=\infty$. Além disso, $\omega(u) \subset B_{\alpha_{0}}(0)$ é compacto, não-vazio e formado por pontos críticos de $I$. No entanto, se $v \in B_{\alpha_{0}}(0)$ for um ponto crítico para $I$, então

$$
\|v\|^{2}=\langle A(v), v\rangle \leq\|v\|^{2}\left(c_{1}+c_{2}\|v\|^{p}\right) \leq\|v\|^{2}\left(c_{1}+c_{2} \alpha_{0}^{p}\right)=\frac{c_{1}+1}{2}\|v\|^{2},
$$

o que é possível somente quando $v=0$, uma vez que $c_{1} \in(0,1)$. Portanto, $\omega(u)=\{0\}$ para todo $u \in B_{r}(0)$. Dessa forma $B_{r}(0) \subset \mathcal{A}_{0}$, o que implica $\mathcal{A}_{0}=\mathcal{A}\left(B_{r}(0)\right)$. Como o fluxo $\varphi$ é uma aplicação contínua, como $B_{r}(0)$ é um aberto, então assim o é $\mathcal{A}\left(B_{r}(0)\right)$. Portanto $\mathcal{A}_{0}$ é uma vizinhança aberta da origem.

Proposição 4.2.5. $\partial \mathcal{A}_{0}$ é um fechado, positivamente invariante $e \inf _{u \in \partial \mathcal{A}_{0}} I(u) \geq 0$. Em particular, para todo $u \in \partial \mathcal{A}_{0}, \omega(u)$ é um compacto não-vazio formado por pontos críticos não-triviais de $I$. 
Demonstração. Obviamente $\partial \mathcal{A}_{0}$ é um conjunto fechado. A demonstração de que $\partial \mathcal{A}_{0}$ é positivamente invariante consta no Lemma 2.3 de [25].

Uma vez que para todo $u \in \mathcal{A}_{0}, I(u) \geq 0$, por continuidade, segue que $I(u) \geq 0$ para todo $u \in \partial \mathcal{A}_{0}$, ou seja $\left\{I(u) ; u \in \partial \mathcal{A}_{0}\right\}$ é limitado inferiormente. Assim, pela Proposição 4.2.2, $\omega(u)$ é não-vazio e formado por pontos críticos não-triviais de $I$, para todo $u \in \partial \mathcal{A}_{0}$.

Pela última proposição, vemos que $\partial \mathcal{A}_{0}$ é um bom lugar para se procurar por soluções nãotriviais de (4.1). Porém, uma vez encontradas, a princípio nada sabemos sobre seu sinal. Para refinar nossa análise devemos introduzir subconjuntos invariantes de $H$, cujas propriedades nos ajudem a localizar as soluções desejadas.

Consideremos então

$$
\begin{gathered}
\mathcal{K}=\{u \in H ; u \geq 0 \quad \text { q.t.p. em } \Omega\} \\
-\mathcal{K}=\{u \in H ; u \leq 0 \quad \text { q.t.p. em } \Omega\},
\end{gathered}
$$

que são cones fechados e convexos. Sejam $P$ e $Q$ as projeções ortogonais de $H$ em $\mathcal{K}$ e $-\mathcal{K}$, respectivamente e $P^{*}$ e $Q^{*}$ dadas por $P^{*}=I d-P$ e $Q^{*}=I d-Q$. Como mostrado no começo desse capítulo, sabemos que para todo $u \in H$

$$
\left\langle P u, P^{*} u\right\rangle=0
$$

e

$$
P^{*} u \in \mathcal{K}^{*},
$$

onde $\mathcal{K}^{*}$ é o cone dual de $\mathcal{K}$ e analogamente para $Q$ e $Q^{*}$.

No nosso argumento, para mostrar a invariância de $\mathcal{K}$ e $-\mathcal{K}$ pelo fluxo $\varphi$, será muito importante mostrar que $\mathcal{K}^{*} \subset-\mathcal{K}$ e que $(-\mathcal{K})^{*} \subset \mathcal{K}$. Isto, por sua vez, será mostrado no próximo resultado, onde se usará o fato de o operador $\left(\Delta^{2}, H^{2} \cap H_{0}^{1}(\Omega)\right)$ gozar de um Princípio do Máximo. Mais especificamente, para todo $h \in L^{\infty}(\Omega), h \geq 0$ e $h \not \equiv 0$, existe uma única solução $v \in H$ de

$$
\begin{cases}\Delta^{2} v & =h \text { em } \Omega \\ v & =\Delta v=0 \text { em } \partial \Omega\end{cases}
$$

e ainda $v \in C^{3}(\bar{\Omega}), v>0$ em $\Omega$ e $\frac{\partial v}{\partial \nu}<0$ em $\partial \Omega$. Isso segue facilmente transformando a equação (4.8) no sistema

$$
\left\{\begin{array}{l}
-\Delta v=u \text { em } \Omega \\
-\Delta u=h \text { em } \Omega \\
u \quad v=0 \text { em } \partial \Omega
\end{array}\right.
$$

e aplicando duas vezes o Princípio do Máximo Forte para $\left(-\Delta, H_{0}^{1}(\Omega)\right)$ e uma vez o Lema de Hopf.

Lema 4.2.6. Se $u \in \mathcal{K}^{*} \backslash\{0\}$, então $u<0$ q.t.p. em $\Omega$. 
Demonstração. Note que $C=\left\{w \in C^{2}(\bar{\Omega}) ; w=0\right.$ em $\left.\partial \Omega\right\}$ é denso em $H$. Então existe $u_{0} \in C$ tal que $\left\langle u, u_{0}\right\rangle>0$. Seja agora $h \in L^{\infty}(\Omega), h \geq 0$ e $h \not \equiv 0$ e $v \in H$ a única solução de (4.8), que como sabemos é regular. Ainda $v>0$ em $\Omega$, bem como $\frac{\partial v}{\partial \nu}<0$ em $\partial \Omega$. Como $\Omega$ é limitado, existe $\epsilon>0$ tal que $v+\epsilon u_{0} \in \mathcal{K}$. Assim,

$$
\begin{aligned}
0 & \geq\left\langle u, v+\epsilon u_{0}\right\rangle \\
& =\langle u, v\rangle+\epsilon\left\langle u, u_{0}\right\rangle \\
& >\langle u, v\rangle \\
& =\int_{\Omega} h u d x .
\end{aligned}
$$

Portanto $u<0$ q.t.p. em $\Omega$.

Observação 4.2.7. Vale a pena observar que, pelo último lema, se $u \in H$ então $u=P u+P^{*} u$, onde $P u \geq 0$ e $P^{*} u \leq 0$ em $\Omega$. Então $u \leq P u$, o que implica $u^{+} \leq P u$. Analogamente, provamos que $P^{*} u \leq u^{-}$, para todo $u \in H$. A mesma argumentação serve para demonstrar que $Q u \leq u^{-}$e $u^{+} \leq Q^{*} u$, para todo $u \in H$.

Lema 4.2.8. O operador A satisfaz as seguintes condições

$\left(A_{3}\right)\langle A(u), v\rangle \leq\left\langle A\left(P^{*} u\right), v\right\rangle$, para todo $u \in H$ e $v \in \mathcal{K}^{*} ;$

$\left(A_{4}\right)\langle A(u), v\rangle \leq\left\langle A\left(Q^{*} u\right), v\right\rangle$, para todo $u \in H$ e $v \in(-\mathcal{K})^{*}$.

Demonstração. Vamos demonstrar somente que $\left(A_{3}\right)$, pois $\left(A_{4}\right)$ segue de maneira análoga.

Como $v \in \mathcal{K}^{*}$, então $v \leq 0$. Por $\left(f_{5}\right)$ e pela Observação 4.2 .7 , segue que

$$
f\left(x, P^{*} u(x)\right) \leq f\left(x, u^{-}(x)\right)
$$

e assim

$$
\begin{aligned}
\langle A(u), v\rangle & =\int_{\Omega} f(x, u) v d x \\
& \leq \int_{\Omega} f\left(x, u^{-}\right) v d x \\
& \leq \int_{\Omega} f\left(x, P^{*} u\right) v d x=\left\langle A\left(P^{*} u\right), v\right\rangle,
\end{aligned}
$$

para todo $u \in H$.

O próximo resultado mostrará que as condições $\left(A_{3}\right)$ e $\left(A_{4}\right)$ implicam que $\mathcal{K}$ e que $-\mathcal{K}$ são invariantes tanto pelo operador $A$, quanto pelo fluxo $\varphi$.

Lema 4.2.9. i) $A(\mathcal{K}) \subset \mathcal{K}$ e $A(-\mathcal{K}) \subset-\mathcal{K}$.

ii) Para $\alpha>0$ suficientemente pequeno, o conjunto $B_{\alpha}(\mathcal{K})$ ( $\alpha$-vizinhança de $\mathcal{K}$ ) é positivamente invariante para $\varphi$. Além disso, todo ponto crítico de $I$ em $\overline{B_{\alpha}(\mathcal{K})}$ está em $\mathcal{K}$. O mesmo resultado vale para o cone $-\mathcal{K}$. 
iii) $\mathcal{K} e-\mathcal{K}$ são positivamente invariantes para $\varphi$.

Demonstração. Toda a demonstração será feita apenas para $\mathcal{K}$, pois para $-\mathcal{K}$ os argumentos são absolutamente os mesmos.

i) Observe que para qualquer $u \in H,\left\|P^{*} u\right\|$ mede a distância entre $u$ e $\mathcal{K}$. Como $A$ satisfaz $\left(A_{3}\right)$, então se $u \in \mathcal{K}$

$$
\begin{aligned}
\left\|P^{*}(A(u))\right\|^{2} & =\langle A(u)-P(A(u)), A(u)-P(A(u))\rangle \\
& =\left\langle A(u), P^{*}(A(u))\right\rangle \\
& \leq\left\langle A\left(P^{*} u\right), P^{*}(A(u))\right\rangle=0 .
\end{aligned}
$$

ii) Seja $u \in H$, por $\left(A_{2}\right)$ e $\left(A_{3}\right)$ temos que

$$
\begin{aligned}
\left\|P^{*}(A(u))\right\|^{2} & =\left\langle A(u), P^{*}(A(u))\right\rangle \\
& \leq\left\langle A\left(P^{*} u\right), P^{*}(A(u))\right\rangle \\
& \leq\left\|P^{*}(A(u))\right\|\left(d_{1}\left\|P^{*} u\right\|+d_{2}\left\|P^{*} u\right\|^{p+1}\right),
\end{aligned}
$$

o que implica, se $\left\|P^{*}(A(u))\right\| \neq 0$,

$$
\left\|P^{*}(A(u))\right\| \leq d_{1}\left\|P^{*} u\right\|+d_{2}\left\|P^{*} u\right\|^{p+1}=\left\|P^{*} u\right\|\left(d_{1}+d_{2}\left\|P^{*} u\right\|^{p}\right) .
$$

Dessa forma, se $0<\left\|P^{*} u\right\|<\left(\frac{1-d_{1}}{2 d_{2}}\right)^{\frac{1}{p}}=: \alpha_{0}$, então

$$
\left\|P^{*}(A(u))<\right\| P^{*} u \| .
$$

Note agora que, se $\alpha<\alpha_{0}$, todo ponto fixo de $A$ em $\overline{B_{\alpha}(\mathcal{K})}$ pertence a $\mathcal{K}$. De fato, se $u \in \overline{B_{\alpha}(\mathcal{K})}$ for ponto fixo de $A$, então ou $u \in \mathcal{K}$ ou $u \in \overline{B_{\alpha}(\mathcal{K})} \backslash \mathcal{K}$. Porém a segunda opção não ocorre pois do contrário, $0<\left\|P^{*} u\right\| \leq \alpha<\alpha_{0}$, de forma que por (4.11)

$$
\left\|P^{*} u\right\|=\left\|P^{*}(A(u))\right\|<\left\|P^{*} u\right\|
$$

o que é uma contradição.

Mostremos agora que $B_{\alpha}(\mathcal{K})$ é positivamente invariante.

Observe que (4.11) implica

$$
A\left(\partial B_{\alpha}(\mathcal{K})\right) \subset \operatorname{int}\left(B_{\alpha}(\mathcal{K})\right)
$$

Suponhamos por contradição que exista $u_{0} \in B_{\alpha}(\mathcal{K})$ tal que $\varphi\left(t_{0}, u_{0}\right) \in \partial B_{\alpha}(\mathcal{K})$, onde $t_{0} \in\left[0, T\left(u_{0}\right)\right)$ é o menos positivo com tal propriedade. Como $B_{\alpha}(\mathcal{K})$ é aberto e convexo e $\left\{\varphi\left(t_{0}, u_{0}\right)\right\}$ é compacto, pelo Teorema de Separação de Mazur seque que existe um funcional linear $\rho \in H^{*}$ e um real $\beta$ tais que $\rho\left(\varphi\left(t_{0}, u_{0}\right)\right)=\beta$ e $\rho(u)>\beta$, para todo $u \in B_{\alpha}(\mathcal{K})$. Note que

$$
\begin{aligned}
\left.\frac{\partial}{\partial t} \rho\left(\varphi\left(t, u_{0}\right)\right)\right|_{t=t_{0}} & =\rho\left(-\nabla I\left(\varphi\left(t_{0}, u_{0}\right)\right)\right) \\
& =\rho\left(A\left(\varphi\left(t_{0}, u_{0}\right)\right)\right)-\beta>0
\end{aligned}
$$


onde foi usado (4.12) na última desigualdade. Com isso, concluímos que existe $\epsilon>0$ tal que $\rho\left(\varphi\left(t, u_{0}\right)\right)<\beta$ para todo $t \in\left(t_{0}-\epsilon, t_{0}\right)$. Assim $\varphi\left(t, u_{0}\right) \notin B_{\alpha}(\mathcal{K})$, para todo $t \in\left(t_{0}-\epsilon, t_{0}\right)$, o que contradiz a minimalidade de $t_{0}$.

iii) Segue do fato do item ii), uma vez lembrado que $\mathcal{K}=\bigcap_{\alpha>0} B_{\alpha}(\mathcal{K})$ e que $-\mathcal{K}=$ $\bigcap_{\alpha>0} B_{\alpha}(-\mathcal{K})$

Seja agora $\alpha>0$ tal que as conclusões do Lema 4.2 .9 valham tanto para $\mathcal{K}$ quanto para $-\mathcal{K}$.

Proposição 4.2.10. Suponha que exista $u_{0} \in \mathcal{K}$ tal que $I\left(u_{0}\right)<0$, então $I$ tem um ponto crítico não-trivial em $\mathcal{K}$. O mesmo resultado vale para $-\mathcal{K}$.

Demonstração. Primeiramente note que pela definição de $\mathcal{A}_{0}$ e pelo fato de $\varphi$ seguir a direção oposta ao crescimento de $I$, segue que $I(u) \geq 0$ para todo $u \in \mathcal{A}_{0}$. Por continuidade, isso se estende naturalmente para todo $u \in \overline{\mathcal{A}_{0}}$.

Como $I\left(u_{0}\right)<0$, naturalmente $u_{0} \notin \overline{\mathcal{A}_{0}}$. Como $\mathcal{A}_{0}$ é uma vizinhança aberta da origem, existe $s \in(0,1)$ tal que $s u_{0} \in \partial \mathcal{A}_{0} \cap \mathcal{K}$. Como $\partial \mathcal{A}_{0} \cap \mathcal{K}$ é um subconjunto fechado e invariante, pela Proposição $4.2 .2, \omega\left(s u_{0}\right) \subset \partial \mathcal{A}_{0} \cap \mathcal{K}$ é não-vazio e qualquer de seus elementos são pontos críticos de $I$.

Denotemos agora por

$$
\mathcal{A}_{+}=\mathcal{A}\left(B_{\alpha}(\mathcal{K})\right) \cap \partial \mathcal{A}_{0} \quad \text { e } \quad \mathcal{A}_{-}=\mathcal{A}\left(B_{\alpha}(-\mathcal{K})\right) \cap \partial \mathcal{A}_{0} .
$$

Lema 4.2.11. $\mathcal{A}_{+}$e $\mathcal{A}_{-}$são abertos relativos de $\partial \mathcal{A}_{0}$ e disjuntos.

Demonstração. Como $B_{\alpha}(\mathcal{K})$ e $B_{\alpha}(-\mathcal{K})$ são abertos, então assim o são $\mathcal{A}\left(B_{\alpha}(\mathcal{K})\right)$ e $\mathcal{A}\left(B_{\alpha}(-\mathcal{K})\right)$. Suponha agora que exista $u \in \mathcal{A}_{+} \cap \mathcal{A}_{-}$. Como $u \in \partial \mathcal{A}_{0}$, então $T(u)=\infty$ e $\omega(u) \neq \emptyset$. Ainda, como $u \in \mathcal{A}\left(B_{\alpha}(\mathcal{K})\right) \cap \mathcal{A}\left(B_{\alpha}(-\mathcal{K})\right)$, então $\omega(u) \subset \overline{B_{\alpha}(\mathcal{K})} \cap \overline{B_{\alpha}(-\mathcal{K})}$. Uma vez que $\omega(u)$ é formado por pontos críticos de $I$, pelo Lema $4.2 .9 \omega(u) \in \mathcal{K} \cap-\mathcal{K}=\{0\}$, o que contraria o fato de $u \in \partial \mathcal{A}_{0}$.

O próximo resultado estabelecerá condições suficientes para a existência de três pontos críticos não-triviais para $I$.

Teorema 4.2.12. Suponha que $\left(A_{1}\right)-\left(A_{4}\right)$ estejam satisfeitas. Suponha ainda que exista um caminho contínuo $h:[0,1] \rightarrow H$ com $h(0) \in \mathcal{K}, h(1) \in-\mathcal{K}$ e $I(h(t))<0$, para todo $t \in[0,1]$. Então $I$ tem ao menos três pontos críticos não-triviais, sendo $u_{1} \in \mathcal{K}, u_{2} \in-\mathcal{K} e$ $u_{3} \in H \backslash(\mathcal{K} \cup-\mathcal{K})$.

Demonstração. A existência de $u_{1}$ e $u_{2}$ segue da Proposição 4.2.10.

Para se obter $u_{3}$, observe primeiramente que $h([0,1]) \cap \overline{\mathcal{A}_{0}}=\emptyset$.

Seja $Q=[0,1]^{2}$ e $\mathcal{B} \subset Q$ definido como 


$$
\mathcal{B}=\left\{\left(s_{1}, s_{2}\right) \in Q ; s_{1} h\left(s_{2}\right) \in \mathcal{A}_{0}\right\}
$$

Observe que $B$ é relativamente aberto em $Q$ e ainda que

- $\{0\} \times[0,1] \subset \mathcal{B}$, pois $0 h(s)=0 \in \mathcal{A}_{0}, \forall s \in[0,1]$

- $\{1\} \times[0,1] \cap \overline{\mathcal{B}}=\emptyset$, pois $I(h(s))<0, \forall s \in[0,1]$.

Usando o Princípio da continuação de Leray-Schauder, pode-se provar que existe uma componente conexa $\Gamma$ de $\partial \mathcal{B}$, tal que

$$
\Gamma \cap([0,1] \times\{0\}) \neq \emptyset \quad \text { e } \quad \Gamma \cap([0,1] \times\{1\}) \neq \emptyset
$$

Considere então $\Sigma$ como o fecho da componente conexa de $\Gamma \backslash \partial Q$ que intersecta $[0,1] \times\{0\}$ $\mathrm{e}[0,1] \times\{1\}$

Seja agora $\Gamma_{0}=\left\{s_{1} h\left(s_{2}\right) ;\left(s_{1}, s_{2}\right) \in \Sigma\right\}$. Então $\Gamma_{0}$ é um subconjunto conexo de $\partial \mathcal{A}_{0}$ tal que $\Gamma_{0} \cap \pm \mathcal{K} \neq \emptyset$. Como pelo Lema 4.2.11, $\mathcal{A}_{ \pm}$são abertos disjuntos de $\partial \mathcal{A}_{0}$, então $\Gamma_{0} \cap \mathcal{A}_{ \pm}$ são abertos disjuntos de $\Gamma_{0}$. Pela conexidade de $\Gamma_{0}$, existe $u \in \Gamma_{0} \backslash\left(\mathcal{A}_{+} \cup \mathcal{A}_{-}\right)$. Uma vez que $\partial \mathcal{A}_{0} \backslash\left(\mathcal{A}_{+} \cup \mathcal{A}_{-}\right)$é invariante, então $\{\varphi(t, u) ; t \geq 0\} \subset \partial \mathcal{A}_{0} \backslash\left(\mathcal{A}_{+} \cup \mathcal{A}_{-}\right)$. Como esse conjunto é fechado em $\partial \mathcal{A}_{0}$, então $\omega(u) \subset \partial \mathcal{A}_{0} \backslash\left(\mathcal{A}_{+} \cup \mathcal{A}_{-}\right)$. Em particular $\omega(u) \cap(\mathcal{K} \cup-\mathcal{K}) \neq \emptyset$ e assim, qualquer de seus elementos tem a propriedade enunciada para $u_{3}$.

O próximo resultado será importante para enquadrar o funcional $I$ nas condições do teorema anterior.

Lema 4.2.13. Se $S \subset H \backslash\{0\}$ é compacto e $\tilde{S}=\{t u ; u \in S$ e $t \geq 0\}$, então

$$
I(u) \rightarrow-\infty, \quad \text { quando } u \in \tilde{S} \text { e }\|u\| \rightarrow \infty
$$

Demonstração. Por $\left(f_{3}\right)$, dado $M>0$ qualquer, existe $K_{M}>0$ tal que

$$
F(x, s) \geq M s^{2} \quad \text { se }|s|>K_{M}
$$

Considerando $C_{M}=\max \left\{0, \max _{|s| \geq K_{M}}\left(M s^{2}-F(x, s)\right)\right\}$, vale então que

$$
F(x, s) \geq M s^{2}-C_{M} \quad \text { para todo } s \in \mathbb{R} .
$$

Seja agora $\left\{u_{n}\right\} \subset \tilde{S}$ uma sequência tal que $\left\|u_{n}\right\| \rightarrow \infty$. Então $u_{n}=t_{n} v_{n}$, onde $t_{n} \geq 0$ e $v_{n} \in S$. Como $S$ é compacto, podemos supor que a menos de subsequência $v_{n} \rightarrow v$, quando $n \rightarrow \infty$, para algum $v \in S$. Como $\left\|u_{n}\right\| \rightarrow \infty$, então obviamente $t_{n} \rightarrow \infty$.

Temos então 


$$
\begin{aligned}
I\left(u_{n}\right) & =I\left(t_{n} v_{n}\right) \\
& =t_{n}^{2} \frac{\left\|v_{n}\right\|^{2}}{2}-\int_{\Omega} F\left(x, t_{n} v_{n}\right) d x \\
& \leq t_{n}^{2} \frac{\left\|v_{n}\right\|^{2}}{2}-\int_{\Omega}\left(M t_{n}^{2} v_{n}^{2}-C_{M}\right) d x \\
& =\frac{t_{n}^{2}}{2}\left(\left\|v_{n}\right\|^{2}-2 M\left\|v_{n}\right\|_{L^{2}(\Omega)}^{2}-\frac{C_{M}|\Omega|}{t_{n}^{2}}\right) \\
& =\frac{t_{n}^{2}}{2}\left(\|v\|^{2}-2 M\|v\|_{L^{2}(\Omega)}^{2}+o_{n}(1)\right) .
\end{aligned}
$$

Dessa forma, é possível escolher $M>0$ grande o suficiente para que $\|v\|^{2}-2 M\|v\|_{L^{2}(\Omega)}^{2}<0$ e assim

$$
I\left(u_{n}\right) \rightarrow-\infty \text { quando } n \rightarrow \infty
$$

Vamos agora finalmente provar o resultado principal desse capítulo.

Demonstração do Teorema 4.0.3. Seja $u \in \mathcal{K}$ e $v \in \mathcal{K}^{*}$, com $u, v \neq 0$ e $u$ e $v$ vetores de $H$ linearmente independentes. Para cada $s>0$, defina $h_{s}:[0,1] \rightarrow H$, por $h_{s}(t)=$ $s(t u+(1-t) v)$.

Observe que, para todo $s>0$,

- $h_{s}(1)=s u \in \mathcal{K} \backslash\{0\}$,

- $h_{s}(0)=s v \in \mathcal{K}^{*} \backslash\{0\}$.

Pelo Lema 4.2.13 aplicado ao compacto $S=\{t u+(1-t) v ; t \in[0,1]\}$, vemos que se $s$ é suficientemente grande, $I\left(h_{s}(t)\right)<0, \forall t \in[0,1]$. Assim o Teorema 4.2 .12 nos dá o resultado. 



\section{Referências Bibliográficas}

[1] Adams, R. Sobolev Spaces, Pure and Applied Mathematics, Academic Press (1975).

[2] Agmon, S. The $L^{p}$ approach to the Dirichlet problem, Ann. Scuola Norm. Sup. Pisa 13 (1959), $405-448$.

[3] Alves, C., Figueiredo, G. Existence and multiplicity of positive solutions to a pLaplacian equation in $\mathbb{R}^{N}$, Differential and Integral Equations, Vol. 19, No 2 (2006), $143-162$.

[4] Alves, C. Multiplicity of multi-bump type nodal solutions for a class of elliptic problems in $\mathbb{R}^{N}$, Topological Methods in Nonlinear Analysis, Vol. 34 (2009), 231 $-250$.

[5] Alves, C. Existence of multi-peak solutions for a class of quasilinear problems in $\mathbb{R}^{N}$, to appear in Topological Methods in Nonlinear Analysis.

[6] Alves, C., Figueiredo, G. On multiplicity and concentration of positive solutions for a class of quasilinear problems with critical exponential growth in $\mathbb{R}^{N}$, Journal of Differential Equations, Vol. 246 (2009), 1288 - 1311.

[7] Alves, C., Do Ó, J. M., Mryagaki, O. H. Nontrivial solutions for a class of semilinear biharmonic problems involving critical exponents, Nonlinear Analysis, Vol. 46 (2001), 121 - 133.

[8] Alves, C., do Ó, J. M., Miyagaki, O. H. On a class of singular biharmonic problems involving critical exponents, Journal of Mathematical Analysis and Applications, Vol. 227 (2003), 12 - 26.

[9] Alves, C., Souto, M., Local moutain-pass for a class of elliptic problems in $\mathbb{R}^{N}$ involving critical growth, Nonlinear Analysis, Vol. 46 (2001), 495 - 510. 
[10] Alves, C., Sonres, S. On the location and profile of spike-layer nodal solutions to nonlinear Schödinger equations, Journal of Mathematical Analysis and Applications, Vol. 296 (2004), 563 - 577.

[11] Alves, C., Soares, S. Nodal solutions for singularly perturbed equations with critical exponential growth, Journal of Differential Equations, No. 234 (2007), 464 - 484.

[12] Alves, C., Sonres, S. Multiplicity of positive solutions for a class of nonlinear Schrödinger equations, Advances in Differential Equations, Vol. 15, No. 11 - 12 (2010), 1083 - 1102.

[13] Bartsch, T., WAng, Z. Multiple positive solutions for a nonlinear Schrödinger equation, ZAMP, Vol. 51 (2000), 366 - 384.

[14] Bernis, F., Garcia-Azorero, J., Peral, I. Existence and multiplicity of nontrivial solutions in semilinear critical problems of fourth order, Advances in Differeitial Equations, Vol. 1, No 2 (1996), 219 - 240.

[15] BRÄU, T. A decomposition method with respect to dual cones and its application to higher order Sobolev spaces, Download: http://www-ian.math.unimagdeburg.de/home/grunau/papers/BraeuStudEnglisch.pdf.

[16] Brezis, H., Lieb, E. A relation between pointwise convergence of functions and convergence of functionals, Proc. Amer. Math. Soc. Vol. 88, no. 3 (1983), 486 - 490.

[17] Chabrowski, J., Do Ó, J. M. On some fourth-order semilinear elliptic problems in $\mathbb{R}^{N}$, Nonlinear Analysis, Vol. 49, Issue 6 (2002), 861 - 884.

[18] Del Pino, M., Felmer, P. Local mountain pass for semilinear elliptic problems in unbounded domains, Calc. Var. Partial Differential Equations, Vol. 4 (1996), 121 137.

[19] Ding, P., Tanaka, K. Multiplicity of positive solutions of a nonlinear Schrödinger equation, Manuscripta Math., Vol. 112 (2003), 109 - 135.

[20] Floer, A., Weinstein, A. Nonspreading wave pachets for the cubic Schrödinger equations with potential, Journal of Functional Analysis, Vol. 69 (1986), 397 - 408.

[21] Gazzola, F., Grunau, H., Sweers, G. Polyharmonic boundary value problems, Lectures notes in mathematics, Springer (2010).

[22] Jeanjean, L., Tanaka, K. Singularly perturbed elliptic problems with superlinear or asymptotically linear nonlinearities, Calculus of Variations, Vol.21 (2004), 287 318.

[23] Lions, P. L. The concentration compactness principle in the calculus of variations. The locally compact case, Part 2, Analyse Nonlin., Vol. 1 (1984), 223 - 283. 
[24] LiU, X., HuAng, Y. On sign-changing solution for a four-order assymptotically linear elliptic problem, Nonlinear analysis, Vol. 72 (2010), 2271 - 2276.

[25] Liu, Z., Sun, J. Invariant sets of descending flow in critical point theory with applications to nonlinear differential equations, Journal of Differential Equations, Vol. 172 (2001), 257 - 299.

[26] Mawhin, J., Willem, M. Critical point theory and hamiltonean systems, Springer Verlag (1989).

[27] Miyagaki, O., Souto, M. Superlinear problems without Ambrosetti and Rabinowitz growth condition, Journal of Differential Equations, No. 245 (2008), 3628 - 3638.

[28] Moreau, J. Décomposition orthogonale d'un espace hilbertien selon deux cônes mutuellement polaires, C. R. Acad. Sci. Paris, Vol. 255 (1962), 238 - 240.

[29] Он, Y. G. Existence of semi-classical bound states of nonlinear Schrödinger equations with potentials of the class $(V)_{a}$, Communications in Partial Differential Equations, Vol. 13 (1988), 1499 - 1519.

[30] Rabinowitz, P. H. On a class of nonlinear Schrödinger equations, ZAMP, Vol.43 (1992), $270-291$.

[31] Ramos, M. Uniform estimates for the biharmonic operator in $\mathbb{R}^{N}$ and applications, Commun. Appl. Analysis, Vol.8, No. 4 (2009), 435 - 457.

[32] Salvatore, A., Squassina, M. Deformation from symmetry for Schrödinger equations of higher order on unbounded domains, Electronic Journal of Differential Equations, No. 65 (2003), 1 - 15.

[33] Szulkin, A., Weth, T. The method of Nehari manifold, Handbook of Nonconvex Analysis and Applications, D.Y. Gao and D. Motreanu eds., International Press, Boston (2010), 597 - 632.

[34] Trudinger, N., Gilbarg, D. Elliptic Partial Differential Equations of Second Order, Springer (1998).

[35] WANG, X. On concentration of positive bound states of nonlinear Schrödinger equations, Communications in mathematical Physics, Vol. 153 (1993), 229 - 244.

[36] Weth, T. Nodal solutions to superlinear biharmonic equations via decomposition in dual cones, Topological methods in nonlinear analysis, Vol. 28 (2006), 33 - 52.

[37] Willem, M. Minimax theorems, Birkhüser (1996).

[38] Yang, J., Chabrowski, J. Nonnegative solutions for semilinear biharmonic equations in $\mathbb{R}^{N}$, Analysis, Vol. 17 (1997), 35 - 59. 
